\title{
O new physics, where art thou? A global search in the top sector
}

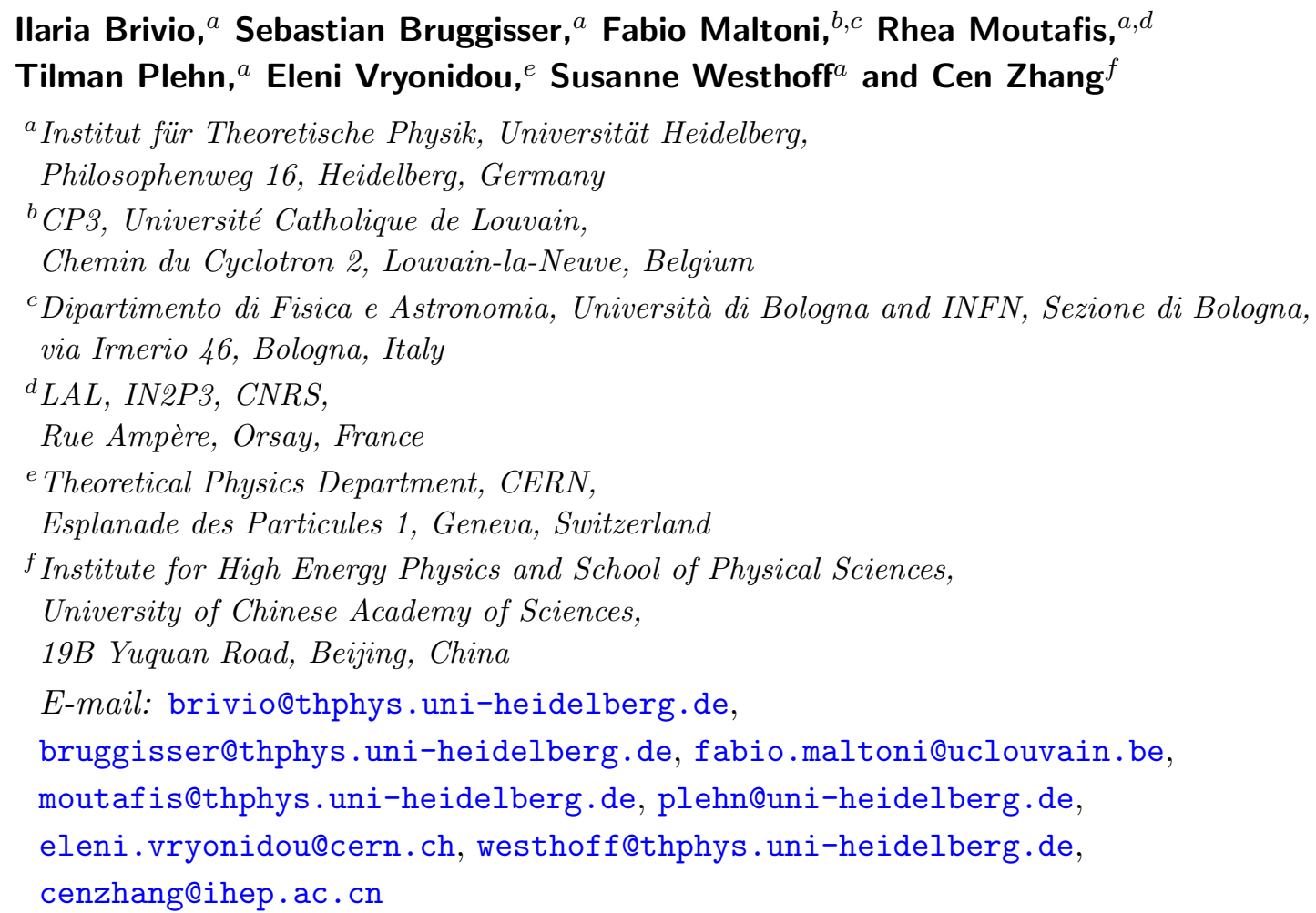

ABSTRACT: We provide a comprehensive global analysis of Run II top measurements at the LHC in terms of dimension- 6 operators. A distinctive feature of the top sector as compared to the Higgs-electroweak sector is the large number of four-quark operators. We discuss in detail how they can be tested and how quadratic terms lead to a stable limit on each individual Wilson coefficient. Predictions for all observables are computed at NLO in QCD. Our SFITTER analysis framework features a detailed error treatment, including correlations between uncertainties.

KeYwords: Beyond Standard Model, Effective Field Theories, Heavy Quark Physics

ARXIV EPRINT: 1910.03606 


\section{Contents}

1 Introduction $\quad 1$

2 Top-quark effective theory 3

2.1 Top pair production 5

2.2 Single top production and top decay 9

2.3 Associated $t \bar{t} W$ and $t \bar{t} Z$ production 12

3 Global analysis setup 13

3.1 Data set 13

$\begin{array}{lll}3.2 & \text { SFitter analysis } & 15\end{array}$

4 Top pair features $\quad 17$

$\begin{array}{lll}4.1 & \text { Event kinematics } & 17\end{array}$

4.2 Incoming up versus down quarks 20

4.3 Top chirality from charge asymmetry 23

4.4 Top chirality from jet radiation 25

4.5 Quadratic terms and flat directions 26

5 Single top analysis $\quad 29$

6 Global top analysis $\quad 31$

7 Conclusions $\quad 34$

$\begin{array}{ll}\text { A Operator relations } & 35\end{array}$

B Numerical bounds on operators $\quad 36$

\section{Introduction}

Contemporary interpretation frameworks for LHC measurements are driven by the structural completeness of the Standard Model (SM) with a light observed Higgs boson and missing hints to the nature of physics beyond the SM. From a quantum field theory perspective the natural approach is therefore to consider the SM as an effective field theory (SMEFT). Here the effects of potential new particles can be systematically included in terms of higher-dimensional operators, suppressed by a sufficiently large matching scale. The framework relies on the idea that new physics affecting LHC measurements is too heavy to be produced and observed directly. This is a direct application of the general 
condition that an effective Lagrangian is applicable if the additional contributing degrees of freedom are kinematically decoupled.

Part of the SMEFT framework [1-5] was developed as a gauge-invariant description of anomalous gauge boson interactions at LEP $[6,7]$. Its biggest success has been the application to Higgs and electroweak boson measurements at the LHC [8-14]. Most recently, the same approach [15-17] has been used to systematically analyze top quark measurements at the LHC [18-24] and at future colliders [25, 26] and their link to bottom sector [27]. These efforts pave the way towards a global SMEFT analysis at the LHC.

Searches for physics beyond the Standard Model in the top sector reflect three unique aspects of top physics: first, top physics at the LHC has entered a phase of precision predictions and measurements, a development which many of us would not have thought to be feasible before the start of the LHC; second, we might start to doubt available solutions of the hierarchy problem, but from a field theory perspective it has not lost its appeal and it singles out the top sector; third, many new physics scenarios, either weakly or strongly interacting at scales around a few $\mathrm{TeV}$, predict deviations in the top couplings or new top interactions, such as new scalars coupled dominantly to the top quark [28].

In this paper we present a comprehensive analysis of the top sector in the framework of SMEFT, based on the data collected mostly during the LHC Run II. We consider measurements in top pair production, including associated $t \bar{t} W$ and $t \bar{t} Z$ production, as well as in single top final states. Since effective interactions typically have a sizeable impact on kinematic distributions we add a set of kinematic measurements with a focus on boosted top pair production $[29,30]$. Finally, we include charge asymmetry [15, 31-33] and top decay measurements $[34,35]$ to shed light on specific sectors of the effective Lagrangian. To combine all of these measurements in a coherent picture of the top sector a global SMEFT analysis is without alternative. Our enlarged set of observables builds on earlier analyses by the TopFitter $[19,20]$ collaboration.

For our simulations we rely on next-to-leading order (NLO) QCD predictions obtained through FeynRules [36], NLOCT [37] and MadGraph5_AMC@NLO [36]. NLO predictions allow us to better control the accuracy and theoretical precision of our predictions, i.e., the theoretical uncertainties in our fit. This is especially important in phase space regions where SMEFT contributions can be large, e.g., in tails of kinematic distributions. For the global analysis we use the established SFITTER framework $[38,39]$. Our technical focus is on the consistent treatment of different types of uncertainties, including correlations of systematic and theoretical uncertainties. The specific error treatment, a detailed analysis of several physics aspects not considered before, as well as a slightly different data set complement other state-of-the-art analyses, such as that of the SMEFiT collaboration [23]. In particular, the SFITTER technology allows us to easily study correlations between Wilson coefficients and to compare the impact of theoretical and experimental uncertainties.

The paper is organized as follows. We begin with an overview of the observables used in our fit in section 2, where we derive analytic expressions for the operator contributions. After recapitulating the main aspects of the fitting approach in section 3 we describe some of the unique aspects of a SMEFT analysis of top pair production in section 4. They are related to the fact that many features of the set of four-quark operators are not immediately 
distinguishable in QCD processes. A crucial aspect is that while flat directions exist in this sector, the quadratic contributions from dimension- 6 operators turn them into compact circular correlations. This in turn allows us to derive limits from profile likelihoods. Finally, we perform a global fit first of the single top sector in section 5 and then of the entire top sector in section 6. Details about our choice of operators and our numerical results are discussed in the appendix.

\section{Top-quark effective theory}

In the absence of new resonances, effects of new physics can be described as effective interactions of SM particles at energies below a new physics matching scale $\Lambda$. Our goal is to probe effective interactions with top quarks in LHC observables [40]. The dominant effects are parametrized in terms of Wilson coefficients $C_{k}$ of dimension- 6 operators $O_{k}$ in the effective Lagrangian

$$
\mathcal{L}_{\text {eff }}=\sum_{k}\left(\frac{C_{k}}{\Lambda^{2}} O_{k}+\text { h.c. }\right)+\sum_{l} \frac{C_{l}}{\Lambda^{2}} O_{l},
$$

where the sum runs over all operators that involve top-quarks. Non-hermitian operators are denoted as ${ }^{\ddagger} O$. We neglect operators of mass dimension seven and higher in the EFT expansion. We focus on CP-conserving extensions of the SM, assuming that all Wilson coefficients are real and therefore neglecting CP-violating interactions. Since top-quark observables at the LHC are largely blind to the flavor of light quarks with the same quantum numbers, we impose an $\mathrm{U}(2)_{q} \times \mathrm{U}(2)_{u} \times \mathrm{U}(2)_{d}$ flavor symmetry among quarks of the first and second generation [40-42]. We use

$$
\begin{aligned}
q_{i} & =\left(u_{L}^{i}, d_{L}^{i}\right) \quad u_{i}=u_{R}^{i}, d_{i}=d_{R}^{i} \quad i=1,2 \\
Q & =\left(t_{L}, b_{L}\right) \quad t=t_{R}, b=b_{R}
\end{aligned}
$$

to denote left- and right-handed quarks of the first two generations and the third generation, respectively. Within this framework, we consider 22 independent operators:

- 8 four-quark operators with $L L$ and $R R$ chiral structure

$$
\begin{aligned}
O_{Q q}^{1,8} & =\left(\bar{Q} \gamma_{\mu} T^{A} Q\right)\left(\bar{q}_{i} \gamma^{\mu} T^{A} q_{i}\right) & & O_{Q q}^{1,1}=\left(\bar{Q} \gamma_{\mu} Q\right)\left(\bar{q}_{i} \gamma^{\mu} q_{i}\right) \\
O_{Q q}^{3,8} & =\left(\bar{Q} \gamma_{\mu} T^{A} \tau^{I} Q\right)\left(\bar{q}_{i} \gamma^{\mu} T^{A} \tau^{I} q_{i}\right) & O_{Q q}^{3,1} & =\left(\bar{Q} \gamma_{\mu} \tau^{I} Q\right)\left(\bar{q}_{i} \gamma^{\mu} \tau^{I} q_{i}\right) \\
O_{t u}^{8} & =\left(\bar{t} \gamma_{\mu} T^{A} t\right)\left(\bar{u}_{i} \gamma^{\mu} T^{A} u_{i}\right) & O_{t u}^{1} & =\left(\bar{t} \gamma_{\mu} t\right)\left(\bar{u}_{i} \gamma^{\mu} u_{i}\right) \\
O_{t d}^{8} & =\left(\bar{t} \gamma^{\mu} T^{A} t\right)\left(\bar{d}_{i} \gamma_{\mu} T^{A} d_{i}\right) & O_{t d}^{1} & =\left(\bar{t} \gamma^{\mu} t\right)\left(\bar{d}_{i} \gamma_{\mu} d_{i}\right) ;
\end{aligned}
$$

- 6 four-quark operators with $L R$ and $R L$ chiral structure

$$
\begin{aligned}
O_{Q u}^{8} & =\left(\bar{Q} \gamma^{\mu} T^{A} Q\right)\left(\bar{u}_{i} \gamma_{\mu} T^{A} u_{i}\right) & O_{Q u}^{1} & =\left(\bar{Q} \gamma^{\mu} Q\right)\left(\bar{u}_{i} \gamma_{\mu} u_{i}\right) \\
O_{Q d}^{8} & =\left(\bar{Q} \gamma^{\mu} T^{A} Q\right)\left(\bar{d}_{i} \gamma_{\mu} T^{A} d_{i}\right) & O_{Q d}^{1} & =\left(\bar{Q} \gamma^{\mu} Q\right)\left(\bar{d}_{i} \gamma_{\mu} d_{i}\right) \\
O_{t q}^{8} & =\left(\bar{q}_{i} \gamma^{\mu} T^{A} q_{i}\right)\left(\bar{t} \gamma_{\mu} T^{A} t\right) & O_{t q}^{1} & =\left(\bar{q}_{i} \gamma^{\mu} q_{i}\right)\left(\bar{t} \gamma_{\mu} t\right) ;
\end{aligned}
$$


- 8 operators with two heavy quarks and bosons [43]

$$
\begin{aligned}
& O_{\phi Q}^{1}=\left(\phi^{\dagger} i \overleftrightarrow{D_{\mu}} \phi\right)\left(\bar{Q} \gamma^{\mu} Q\right) \quad{ }^{\ddagger} O_{t B}=\left(\bar{Q} \sigma^{\mu \nu} t\right) \widetilde{\phi} B_{\mu \nu} \\
& O_{\phi Q}^{3}=\left(\phi^{\dagger} i \overleftrightarrow{D_{\mu}^{I}} \phi\right)\left(\bar{Q} \gamma^{\mu} \tau^{I} Q\right) \quad{ }^{\ddagger} O_{t W}=\left(\bar{Q} \sigma^{\mu \nu} t\right) \tau^{I} \widetilde{\phi} W_{\mu \nu}^{I} \\
& O_{\phi t}=\left(\phi^{\dagger} i \overleftrightarrow{D_{\mu}} \phi\right)\left(\bar{t} \gamma^{\mu} t\right) \quad \ddagger O_{b W}=\left(\bar{Q} \sigma^{\mu \nu} b\right) \tau^{I} \phi W_{\mu \nu}^{I} \\
& { }^{\ddagger} O_{\phi t b}=\left(\widetilde{\phi}^{\dagger} i D_{\mu} \phi\right)\left(\bar{t} \gamma^{\mu} b\right) \quad{ }^{\ddagger} O_{t G}=\left(\bar{Q} \sigma^{\mu \nu} T^{A} t\right) \widetilde{\phi} G_{\mu \nu}^{A} \text {. }
\end{aligned}
$$

The different color structures of the operators will eventually lead to different color factors in the LHC observables and different limits on the Wilson coefficients, as we will see later. In appendix A, we list the relations between these operators and the operators in the Warsaw basis [44]. Gauge invariance imposes relations between effective top couplings to gauge bosons. We define

$$
\begin{array}{ll}
C_{\phi Q}^{-} \equiv C_{\phi Q}^{1}-C_{\phi Q}^{3} & C_{t Z} \equiv-s_{w} C_{t B}+c_{w} C_{t W} \\
C_{\phi Q}^{+} \equiv C_{\phi Q}^{1}+C_{\phi Q}^{3}=C_{\phi Q}^{-}+2 C_{\phi Q}^{3} & C_{t A} \equiv c_{w} C_{t B}+s_{w} C_{t W}=\frac{1}{s_{w}}\left(C_{t W}-c_{w} C_{t Z}\right),
\end{array}
$$

We choose $C_{\phi Q}^{3}, C_{\phi Q}^{-}$and $C_{t W}, C_{t Z}$ as degrees of freedom in our analysis. With this choice, $C_{\phi Q}^{-}$and $C_{t Z}$ modify the $t \bar{t} Z$ coupling, $C_{t W}$ modifies the $t b W$ vertex, while $C_{\phi Q}^{3}$ affects $t b W$ and $b \bar{b} Z$ vertices.

The Wilson coefficients of the operators in eqs. (2.3), (2.4), and (2.5) define the 22 parameters in our global analysis. Further operators either do not leave visible effects in the observables we have selected (like operators with four heavy quarks) or are strongly constrained by more sensitive observables (like the Yukawa operator at dimension six, which is constrained by Higgs measurements). We therefore do not include them in our analysis, but mention them whenever they are relevant.

Experimentally, we focus on observables in top pair and electroweak single top production at the LHC. These processes are precisely predicted and measured, both at the level of total rates and kinematic distributions. We also include top pair production in association with a $W$ - or $Z$-boson, which is more sensitive to certain operators than top pair or single top production and help distinguishing between operators. Table 1 shows our set of Wilson coefficients and their contributions to the various processes.

In what follows, we describe in detail how the 22 top operators affect these processes. We include contributions to $\mathcal{O}\left(\Lambda^{-2}\right)$ when calculating the amplitude of a process and we retain effects of $\mathcal{O}\left(\Lambda^{-4}\right)$, stemming from squaring the $\mathcal{O}\left(\Lambda^{-2}\right)$ amplitude. These contributions will play a crucial role in our global fit. A full calculation of observables at order $\Lambda^{-4}$ in the EFT is beyond the scope of our work.

Another important aspect in our discussion is the energy dependence of operator contributions, which changes the top kinematics in distributions. For top pair production, we present complete analytic expressions for four-quark operator contributions at LO, including both SM-interference and dimension-6 squared terms. 


\begin{tabular}{|l|ccccccc|}
\hline parameter & $t \bar{t}$ & single $t$ & $t W$ & $t Z$ & $t$ decay & $t \bar{t} Z$ & $t \bar{t} W$ \\
\hline$C_{Q q}^{1,8}$ & $\Lambda^{-2}$ & - & - & - & - & $\Lambda^{-2}$ & $\Lambda^{-2}$ \\
$C_{Q q}^{3,8}$ & $\Lambda^{-2}$ & $\Lambda^{-4}\left[\Lambda^{-2}\right]$ & - & $\Lambda^{-4}\left[\Lambda^{-2}\right]$ & $\Lambda^{-4}\left[\Lambda^{-2}\right]$ & $\Lambda^{-2}$ & $\Lambda^{-2}$ \\
$C_{t u}^{8}, C_{t d}^{8}$ & $\Lambda^{-2}$ & - & - & - & - & $\Lambda^{-2}$ & - \\
$C_{Q q}^{1,1}$ & $\Lambda^{-4}\left[\Lambda^{-2}\right]$ & - & - & - & - & $\Lambda^{-4}\left[\Lambda^{-2}\right]$ & $\Lambda^{-4}\left[\Lambda^{-2}\right]$ \\
$C_{Q q}^{3,1}$ & $\Lambda^{-4}\left[\Lambda^{-2}\right]$ & $\Lambda^{-2}$ & - & $\Lambda^{-2}$ & $\Lambda^{-2}$ & $\Lambda^{-4}\left[\Lambda^{-2}\right]$ & $\Lambda^{-4}\left[\Lambda^{-2}\right]$ \\
$C_{t u}^{1}, C_{t d}^{1}$ & $\Lambda^{-4}\left[\Lambda^{-2}\right]$ & - & - & - & - & $\Lambda^{-4}\left[\Lambda^{-2}\right]$ & - \\
\hline$C_{Q u}^{8}, C_{Q d}^{8}$ & $\Lambda^{-2}$ & - & - & - & - & $\Lambda^{-2}$ & - \\
$C_{t q}^{8}$ & $\Lambda^{-2}$ & - & - & - & - & $\Lambda^{-2}$ & $\Lambda^{-2}$ \\
$C_{Q u}^{1}, C_{Q d}^{1}$ & $\Lambda^{-4}\left[\Lambda^{-2}\right]$ & - & - & - & - & $\Lambda^{-4}\left[\Lambda^{-2}\right]$ & - \\
$C_{t q}^{1}$ & $\Lambda^{-4}\left[\Lambda^{-2}\right]$ & - & - & - & - & $\Lambda^{-4}\left[\Lambda^{-2}\right]$ & $\Lambda^{-4}\left[\Lambda^{-2}\right]$ \\
\hline$C_{\phi Q}^{-}$ & - & - & - & $\Lambda^{-2}$ & - & $\Lambda^{-2}$ & - \\
$C_{\phi Q}^{3}$ & - & $\Lambda^{-2}$ & $\Lambda^{-2}$ & $\Lambda^{-2}$ & $\Lambda^{-2}$ & $\Lambda^{-2}$ & - \\
$C_{\phi t}$ & - & - & - & $\Lambda^{-2}$ & - & $\Lambda^{-2}$ & - \\
$C_{\phi t b}$ & - & $\Lambda^{-4}$ & $\Lambda^{-4}$ & $\Lambda^{-4}$ & $\Lambda^{-4}$ & - & - \\
$C_{t Z}$ & - & - & - & $\Lambda^{-2}$ & - & $\Lambda^{-2}$ & - \\
$C_{t W}$ & - & $\Lambda^{-2}$ & $\Lambda^{-2}$ & $\Lambda^{-2}$ & $\Lambda^{-2}$ & - & - \\
$C_{b W}$ & - & $\Lambda^{-4}$ & $\Lambda^{-4}$ & $\Lambda^{-4}$ & $\Lambda^{-4}$ & - & - \\
$C_{t G}$ & $\Lambda^{-2}$ & {$\left[\Lambda^{-2}\right]$} & $\Lambda^{-2}$ & - & {$\left[\Lambda^{-2}\right]$} & $\Lambda^{-2}$ & $\Lambda^{-2}$ \\
\hline
\end{tabular}

Table 1. Wilson coefficients in our analysis and their contributions to top-quark observables via SM-interference $\left(\Lambda^{-2}\right)$ and via dimension-6 squared terms only $\left(\Lambda^{-4}\right)$. A square bracket indicates that the Wilson coefficient contributes via SM-interference at NLO QCD. All quark masses except $m_{t}$ are assumed to be zero. 'Single $t$ ' stands for $s$ - and $t$-channel electroweak top production.

\subsection{Top pair production}

Hadronic top pair production involves $g g \rightarrow t \bar{t}$ and $q \bar{q} \rightarrow t \bar{t}$ partonic processes. In SMEFT, effective operators contribute to both processes, as shown in figure 1. At the LHC, top pair production is dominated by incoming gluons. At leading order in QCD two operators contribute to this rate,

$$
{ }^{\ddagger} O_{t G}=\left(\bar{Q} \sigma^{\mu \nu} T^{A} t\right) \widetilde{\phi} G_{\mu \nu}^{A} \quad \text { and } \quad O_{G}=f_{a b c} G_{\mu}^{a \nu} G_{\nu}^{b \rho} G_{\rho}^{c \mu} .
$$

However, $O_{G}$ is strongly constrained by multi-jet production [45, 46],

$$
\frac{\Lambda}{\sqrt{g_{s}\left|C_{G}\right|}}>5.2 \mathrm{TeV} \text {. }
$$

Since this sensitivity is beyond the reach of top pair production, we neglect $O_{G}$ in our analysis. Unfortunately, multi-jet features which lead to this reach in jet production do not help significantly in the top sector $[46,47]$. 

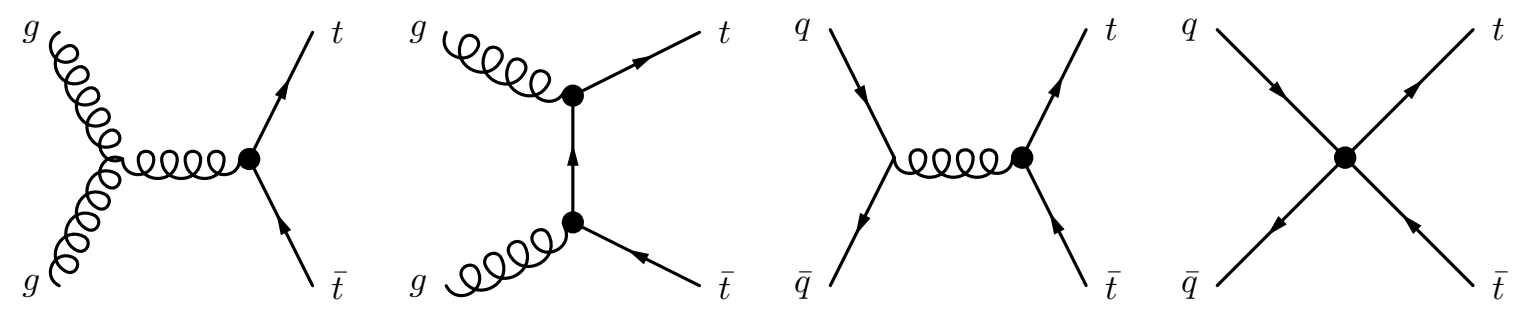

Figure 1. Examples of Feynman diagrams contributing to top pair production in SMEFT at leading order. The dots indicate possible insertions of a dimension- 6 operator.

The contribution of $O_{t G}$ to the partonic differential cross section is given by [16] ${ }^{1}$

$$
\frac{d \sigma(g g \rightarrow t \bar{t})}{d c_{t}}=\frac{\alpha_{s}^{3 / 2} \sqrt{\pi}}{12 \sqrt{2}} \frac{\beta_{t \bar{t}}}{s} \frac{m_{t} v}{\Lambda^{2}} \frac{7+9 \beta_{t \bar{t}}^{2} c_{t}^{2}}{1-\beta_{t \bar{t}}^{2} c_{t}^{2}} C_{t G}+\mathcal{O}\left(\frac{s v^{2}}{\Lambda^{4}} C_{t G}^{2}\right)
$$

where $\beta_{t \bar{t}}=\sqrt{1-4 \bar{m}_{t}^{2}}, \bar{m}_{t}=m_{t} / \sqrt{s}$, and $c_{t}=\cos \theta_{t}$ with the scattering angle $\theta_{t}$ of the top against one of the incoming gluons in the partonic center-of-mass (CM) frame. Due to the large gluon luminosity, we expect a high sensitivity to $O_{t G}$ in inclusive top pair production. At high energies $\sqrt{s} \gg m_{t}$, the $O_{t G}-\mathrm{QCD}$ and $O_{t G}-O_{t G}$ interferences scale as $m_{t} v / \Lambda^{2}$ and $v^{2} s / \Lambda^{4}$ relative to the QCD rate, respectively. In the collinear limit, $c_{t} \approx 1$, $O_{t G}$ contributions feature the same logarithmic enhancement as QCD. The kinematics of $O_{t G^{-}}$QCD interference is thus similar to QCD, while squared $O_{t G}$ contibutions grow with energy relative to the SM. We will discuss the impact of $O_{t G}$ on kinematic distributions in detail in section 4.1.

Compared to the $g g \rightarrow t \bar{t}$ contribution, $q \bar{q}$ scattering is suppressed by the parton luminosities. However, the quark-antiquark luminosity is enhanced in the production of boosted tops, where the partons carry a large fraction of the proton's energy. In this regime, top pair production is most sensitive to the four-quark operators introduced in eqs. (2.3) and (2.4) and their interference with $O_{t G}$.

Contributions of four-quark operators are conveniently classified by their behavior under top charge conjugation. The vector current $V$ is odd under charge conjugation, while the axial-vector current $A$ is even. ${ }^{2}$ We define vector and axial-vector combinations of Wilson coefficients as

$$
\begin{aligned}
& 4 C_{V V}^{u, 8}=C_{Q q}^{1,8}+C_{Q q}^{3,8}+C_{t u}^{8}+C_{t q}^{8}+C_{Q u}^{8} \\
& 4 C_{A A}^{u, 8}=C_{Q q}^{1,8}+C_{Q q}^{3,8}+C_{t u}^{8}-C_{t q}^{8}-C_{Q u}^{8} \\
& 4 C_{A V}^{u, 8}=-\left(C_{Q q}^{1,8}+C_{Q q}^{3,8}\right)+C_{t u}^{8}+C_{t q}^{8}-C_{Q u}^{8} \\
& 4 C_{V A}^{u, 8}=-\left(C_{Q q}^{1,8}+C_{Q q}^{3,8}\right)+C_{t u}^{8}-C_{t q}^{8}+C_{Q u}^{8} .
\end{aligned}
$$

\footnotetext{
${ }^{1}$ Notice that in ref. [16] the top-gluon operator is defined as twice the $O_{t G}$ in eq. (2.5).

${ }^{2}$ Strictly speaking, this statement holds only for the color-singlet currents. The color-octet ones may receive an additional sign under charge conjugation, depending on the color index. This however does not have a consequence in the discussions below, because at the LO the top-quark current is always squared against the same color structure.
} 
Pure $V V, A A, V A$, or $A V$ currents are obtained for

$$
\left|C_{Q q}^{1,8}+C_{Q q}^{3,8}\right|=\left|C_{t u}^{8}\right|=\left|C_{t q}^{8}\right|=\left|C_{Q u}^{8}\right| .
$$

The corresponding combinations with the down-type index $d$ can be derived by replacing the index $u \rightarrow d$ and $+C_{Q q}^{3,8} \rightarrow-C_{Q q}^{3,8}$ in eqs. (2.10) and (2.11). For color-singlet coefficients, we define the same relations by changing all indices $8 \rightarrow 1$ in eq. (2.10), yielding $C_{V V}^{u, 1}$ etc. Neglecting electroweak contributions, the $q \bar{q} \rightarrow t \bar{t}$ partonic rate at LO is then given by $(q=u, d ;$ cf. refs. [16] and [48])

$$
\begin{aligned}
\frac{d \sigma(q \bar{q} \rightarrow t \bar{t})}{d c_{t}}= & \frac{2}{9} \frac{\pi \alpha_{s}^{2} \beta_{t \bar{t}}}{2 s}\left\{\left(1+\beta_{t \bar{t}}^{2} c_{t}^{2}+4 \bar{m}_{t}^{2}\right)+\frac{1}{\sqrt{4 \pi \alpha_{s}}} \frac{m_{t} v}{\Lambda^{2}} \frac{16}{\sqrt{2}} C_{t G}\right. \\
& +\frac{1}{4 \pi \alpha_{s}} \frac{2 s}{\Lambda^{2}}\left[\left(1+\beta_{t \bar{t}}^{2} c_{t}^{2}+4 \bar{m}_{t}^{2}\right) C_{V V}^{q, 8}+2 \beta_{t \bar{t}} c_{t} C_{A A}^{q, 8}\right] \\
& +\frac{1}{\left(4 \pi \alpha_{s}\right)^{2}} \frac{s^{2}}{\Lambda^{4}}\left[4 \beta_{t \bar{t}} c_{t}\left(C_{V V}^{q, 8} C_{A A}^{q, 8}+C_{V A}^{q, 8} C_{A V}^{q, 8}+\frac{9}{2}\left(C_{V V}^{q, 1} C_{A A}^{q, 1}+C_{V A}^{q, 1} C_{A V}^{q, 1}\right)\right)\right. \\
& \left.+\left(1+\beta_{t \bar{t}}^{2} c_{t}^{2}\right)\left(\left|C_{V+A}^{q, 8}\right|^{2}+\frac{9}{2}\left|C_{V+A}^{q, 1}\right|^{2}\right)+4 \bar{m}_{t}^{2}\left(\left|C_{V-A}^{q, 8}\right|^{2}+\frac{9}{2}\left|C_{V-A}^{q, 1}\right|^{2}\right)\right] \\
& \left.+\frac{1}{\left(4 \pi \alpha_{s}\right)^{\frac{3}{2}}} \frac{m_{t} v s}{\Lambda^{4}} \frac{4}{\sqrt{2}}\left(C_{V V}^{q, 8}+\beta_{t \bar{t}} c_{t} C_{A A}^{q, 8}\right) C_{t G}+\mathcal{O}\left(\frac{v^{2} s}{\Lambda^{4}} C_{t G}^{2}\right)\right\},
\end{aligned}
$$

with the combinations of color-octet $(\alpha=8)$ and color-singlet $(\alpha=1)$ Wilson coefficients,

$$
\begin{aligned}
\left|C_{V+A}^{q, \alpha}\right|^{2} & =\left|C_{V V}^{q, \alpha}\right|^{2}+\left|C_{V A}^{q, \alpha}\right|^{2}+\left|C_{A A}^{q, \alpha}\right|^{2}+\left|C_{A V}^{q, \alpha}\right|^{2}, \\
& \stackrel{q=u}{=}\left(\left|C_{Q q}^{1,8}+C_{Q q}^{3,8}\right|^{2}+\left|C_{t u}^{8}\right|^{2}+\left|C_{t q}^{8}\right|^{2}+\left|C_{Q u}^{8}\right|^{2}\right) / 4 \\
\left|C_{V-A}^{q, \alpha}\right|^{2} & =\left|C_{V V}^{q, \alpha}\right|^{2}+\left|C_{V A}^{q, \alpha}\right|^{2}-\left|C_{A A}^{q, \alpha}\right|^{2}-\left|C_{A V}^{q, \alpha}\right|^{2} \\
& \stackrel{q=u}{=}\left(\left(C_{Q q}^{1,8}+C_{Q q}^{3,8}\right) C_{t q}^{8}+C_{t u}^{8} C_{Q u}^{8}\right) / 2 .
\end{aligned}
$$

To understand the operator effects in kinematic distributions, it is instructive to explore their behavior at high CM energies $\sqrt{s}$. The high-energy behavior of the various four-quark contributions and their interference with $O_{t G}$ is summarized in table 2.

Dipole operators like $O_{t G}$ flip the chirality of the top quark and require an insertion of the Higgs vacuum expectation value in the amplitude. Their interference with QCD scales as $m_{t} v / \Lambda^{2}$ and does not feature an enhancement at high energies. Squared $O_{t G}$ contributions, in turn, grow like $s v^{2} / \Lambda^{4}$ as in the $g g \rightarrow t \bar{t}$ process. Four-quark operator interferences with QCD and with $C_{t G}$ grow as $s / \Lambda^{2}$ and $m_{t} v s / \Lambda^{4}$ relative to QCD, respectively. Squared terms in four-quark operators grow even stronger with energy, scaling as $s^{2} / \Lambda^{4}$. The strong enhancement at high energies is typical of a four-quark contact interaction, compared to a short-distance interaction through gluon exchange. Top pair production is thus most sensitive to four-quark operators in the tails of kinematic distributions, due to both a kinematic enhancement and an increased quark-antiquark luminosity.

Among the four-quark interactions, only color-octet operators interfere with QCD and with $C_{t G}$. Color-singlet operators contribute through interference among themselves. The 


\begin{tabular}{|c|c|c|c|c|c|c|}
\hline & $\mathrm{QCD}$ & $C_{t G}$ & $C_{V V}^{q, 8}$ & $C_{A A}^{q, 8}$ & $C_{V V}^{q, 1}$ & $C_{A A}^{q, 1}$ \\
\hline $\mathrm{QCD}$ & 1 & $\frac{m_{t} v}{\Lambda^{2}}$ & $\frac{s}{\Lambda^{2}}$ & $c_{t} \frac{s}{\Lambda^{2}}$ & - & - \\
$C_{t G}$ & $\ldots$ & $\frac{s v^{2}}{\Lambda^{4}}$ & $\frac{s m_{t} v}{\Lambda^{4}}$ & $c_{t} \frac{s m_{t} v}{\Lambda^{4}}$ & - & - \\
$C_{V V}^{q, 8}$ & $\ldots$ & $\ldots$ & $\frac{s^{2}}{\Lambda^{4}}$ & $c_{t} \frac{s^{2}}{\Lambda^{4}}$ & - & - \\
$C_{A A}^{q, 8}$ & $\ldots$ & $\ldots$ & $\ldots$ & $\frac{s^{2}}{\Lambda^{4}}$ & - & - \\
$C_{V V}^{q, 1}$ & $\ldots$ & $\ldots$ & $\ldots$ & $\ldots$ & $\frac{s^{2}}{\Lambda^{4}}$ & $c_{t} \frac{s^{2}}{\Lambda^{4}}$ \\
$C_{A A}^{q, 1}$ & $\ldots$ & $\ldots$ & $\ldots$ & $\ldots$ & $\ldots$ & $\frac{s^{2}}{\Lambda^{4}}$ \\
\hline
\end{tabular}

Table 2. Relative scaling of operator contributions with respect to QCD in top pair production $(q \bar{q} \rightarrow t \bar{t})$ at high energies $\sqrt{s} \gg m_{t}$.

relative factor of $9 / 2$ between quadratic contributions of color-singlet and color-octet operators in eq. (2.12) is due to the color structure. For color singlets, the rate is proportional to the number of colors $N_{c}$ of each quark current, yielding $N_{c}^{2}=9$. For color octets, it is proportional to $\sum_{A B} \operatorname{Tr}\left(T^{A} T^{B}\right)^{2}=\left(N_{c}^{2}-1\right) / 4=2$.

The sensitivity of the $q \bar{q} \rightarrow t \bar{t}$ process to the chirality of the top quarks is crucial to distinguish between different four-quark operators. Their interference with QCD or $O_{t G}$ probes the two combinations $C_{V V}^{q, 8}$ and $C_{A A}^{q, 8}$, i.e., pure vector and axial-vector currents. Interference of two four-quark operators introduces the additional chirality structures from eq. (2.14). The impact of chiral operators on kinematic distributions can be understood by considering charge-symmetric and -asymmetric differential cross sections

$$
\begin{aligned}
d \sigma^{S} & =d \sigma\left(t\left(p_{1}\right) \bar{t}\left(p_{2}\right)\right)+d \sigma\left(t\left(p_{2}\right) \bar{t}\left(p_{1}\right)\right) \\
d \sigma^{A} & =d \sigma\left(t\left(p_{1}\right) \bar{t}\left(p_{2}\right)\right)-d \sigma\left(t\left(p_{2}\right) \bar{t}\left(p_{1}\right)\right),
\end{aligned}
$$

where $p_{1}$ and $p_{2}$ are the 4-momenta of the two tops in the final state. In table 3 , we list the four-quark coefficients contributing to $\sigma^{S}$ and $\sigma^{A}$ in top pair production at LO in QCD. At leading order, 5 chiral combinations of Wilson coefficients per parton contribute. A priori, they can be distinguished by five measurements of different kinematic observables. Charge asymmetries play an important role in this endeavor, since they probe chiral structures that are not accessible in cross sections or other charge-symmetric observables [33]. These observations will be confronted with data in section 4.3, where we show how to use cross sections and asymmetries to gain access to the chirality of the four-quark operators. At $\mathrm{NLO}$, the chiral contributions to $t \bar{t}$ production are modified by QCD corrections, leading to additional kinematic degrees of freedom. In section 4.4, we will elaborate more on NLO effects in kinematic distributions. 


\begin{tabular}{|c|c|}
\hline$\sigma_{k}^{S}$ & $C_{V V}^{q, 8}$ \\
\hline$\sigma_{k l}^{S}$ & $\left|C_{V+A}^{q, 8}\right|^{2}+\frac{9}{2}\left|C_{V+A}^{q, 1}\right|^{2}$ \\
& $\left|C_{V-A}^{q, 8}\right|^{2}+\frac{9}{2}\left|C_{V-A}^{q, 1}\right|^{2}$ \\
\hline$\sigma_{k}^{A}$ & $C_{A A}^{q, 8}$ \\
\hline$\sigma_{k l}^{A}$ & $C_{V V}^{q, 8} C_{A A}^{q, 8}+C_{V A}^{q, 8} C_{A V}^{q, 8}+\frac{9}{2}\left(C_{V V}^{q, 1} C_{A A}^{q, 1}+C_{V A}^{q, 1} C_{A V}^{q, 1}\right)$ \\
\hline
\end{tabular}

Table 3. Four-quark contributions to $t \bar{t}$ production in SMEFT at LO QCD. We separate SMinterference, $\sigma_{k}^{S, A}$, and dimension- 6 squared terms, $\sigma_{k l}^{S, A}$ for charge-symmetric and -asymmetric cross sections.

\subsection{Single top production and top decay}

Single top production and top decay are both sensitive to operators with weak gauge bosons and in this sense complementary to top pair production. We distinguish $t$-channel and $s$ channel production, as well as associated $t W$ and $t Z$ production. Examples of Feynman diagrams for these processes are shown in figure 2.

$t$ - and $s$-channel production probe the same set of operators, because the underlying partonic processes $u b \rightarrow d t$ and $u \bar{d} \rightarrow t \bar{b}$ are related by a crossing symmetry. At the level of SM-interference three dimension-6 operators contribute,

$$
\begin{aligned}
O_{Q q}^{3,1} & =\left(\bar{Q} \gamma_{\mu} \tau^{I} Q\right)\left(\bar{q}_{i} \gamma^{\mu} \tau^{I} q_{i}\right) & { }^{\ddagger} O_{t W}=\left(\bar{Q} \sigma^{\mu \nu} \tau^{I} t\right) \tilde{\phi} W_{\mu \nu}^{I} \\
O_{\phi Q}^{3} & =\left(\phi^{\dagger} \overleftrightarrow{i D_{\mu}^{I}} \phi\right)\left(\bar{Q} \gamma^{\mu} \tau^{I} Q\right) . &
\end{aligned}
$$

Since the kinematics of the two channels is different, we also expect a different sensitivity to these operators. The dominant partonic cross sections for $t$ - and $s$-channel production are given by $[16]$

$$
\begin{aligned}
\frac{d \sigma_{t}(u b \rightarrow d t)}{d c_{t u}}= & \frac{G_{F}^{2} m_{W}^{4} \beta_{t}^{2}}{\pi s\left(2 \bar{m}_{W}^{2}+\beta_{t}\left(1+c_{t u}\right)\right)^{2}}\left\{1+2 \frac{v^{2}}{\Lambda^{2}} C_{\phi Q}^{3}+\sqrt{2} \frac{v^{2}}{\Lambda^{2}} C_{t W} \frac{m_{t}}{m_{W}}\left(1+c_{t u}\right)\right. \\
& \left.-\frac{v^{2}}{\Lambda^{2}} \frac{s}{m_{W}^{2}} C_{Q q}^{3,1}\left(2 \bar{m}_{W}^{2}+\beta_{t}\left(1+c_{t u}\right)\right)\right\} \\
\frac{d \sigma_{s}(u \bar{d} \rightarrow t \bar{b})}{d c_{t u}}= & \frac{G_{F}^{2} m_{W}^{4} \beta_{t}^{2}}{16 \pi s\left(1-\bar{m}_{W}^{2}\right)^{2}}\left(1+c_{t u}\right)\left(1+\beta_{t} c_{t u}+\bar{m}_{t}^{2}\right)\left\{1+2 \frac{v^{2}}{\Lambda^{2}} C_{\phi Q}^{3}\right. \\
& \left.+4 \sqrt{2} \frac{v^{2}}{\Lambda^{2}} C_{t W} \frac{m_{t}}{m_{W}} \frac{1}{1+\beta_{t} c_{t u}+\bar{m}_{t}^{2}}+2 \frac{v^{2}}{\Lambda^{2}} \frac{s}{m_{W}^{2}} C_{Q q}^{3,1}\left(1-\bar{m}_{W}^{2}\right)\right\}
\end{aligned}
$$

Here $\beta_{t}=1-\bar{m}_{t}^{2}, \bar{m}_{W}^{2}=m_{W}^{2} / s$, and $c_{t u}=\cos \theta_{t u}$ is the cosine of the angle between the top and the incoming up quark in the CM system. We set $V_{t b}=1=V_{u d}$ and neglect all quark masses except $m_{t}$. In $t$-channel production, the process $\bar{d} b \rightarrow \bar{u} t$ also contributes, but is subdominant due to the smaller parton luminosity. We do not provide analytic expressions for this channel, but include it in the numerical analysis. 


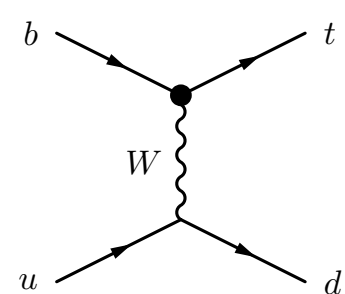

$(t$-channel)

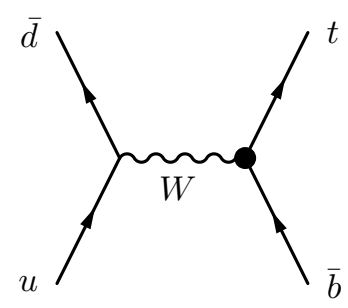

$(s$-channel)

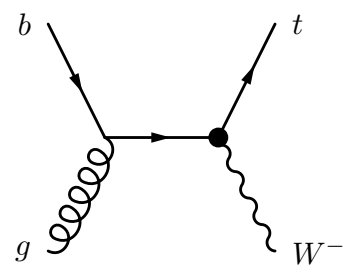

$(t W)$

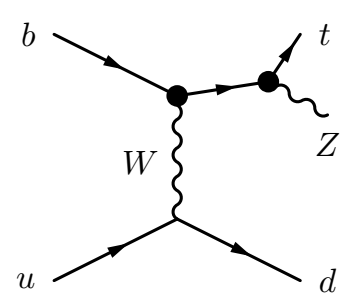

$(t Z)$

Figure 2. Examples of Feynman diagrams contributing to single top production in SMEFT at leading order. The dots indicate possible operator insertions.

\begin{tabular}{|c|c|c|c|c|c|c|c|}
\hline & $\mathrm{SM}$ & $C_{Q q}^{3,1}$ & $C_{\phi Q}^{3}$ & $C_{t W}$ & $C_{Q q}^{3,8}$ & $C_{\phi t b}$ & $C_{b W}$ \\
\hline $\mathrm{SM}$ & 1 & $\frac{m_{W}^{2}}{\Lambda^{2}} \ln \frac{s}{m_{W}^{2}}$ & $\frac{v^{2}}{\Lambda^{2}}$ & $\frac{m_{t} v}{\Lambda^{2}} \frac{m_{W}^{2}}{s} \ln \frac{s}{m_{W}^{2}}$ & - & $\propto m_{b}$ & $\propto m_{b}$ \\
$C_{Q q}^{3,1}$ & $\ldots$ & $\frac{s m_{W}^{2}}{\Lambda^{4}}$ & $\frac{v^{2} m_{W}^{2}}{\Lambda^{4}} \ln \frac{s}{m_{W}^{2}}$ & $\frac{m_{t} v m_{W}^{2}}{\Lambda^{4}}$ & - & $\propto m_{b}$ & $\propto m_{b}$ \\
$C_{\phi Q}^{3}$ & $\ldots$ & $\ldots$ & $\frac{v^{4}}{\Lambda^{4}}$ & $\frac{m_{t} v^{3}}{\Lambda^{4}} \frac{m_{W}^{2}}{s} \ln \frac{s}{m_{W}^{2}}$ & - & $\propto m_{b}$ & $\propto m_{b}$ \\
$C_{t W}$ & $\ldots$ & $\ldots$ & $\ldots$ & $\frac{v^{2} m_{W}^{2}}{\Lambda^{4}}$ & - & $\propto m_{b}$ & $\propto m_{b}$ \\
$C_{Q q}^{3,8}$ & $\ldots$ & $\ldots$ & $\ldots$ & $\ldots$ & $\frac{s m_{W}^{2}}{\Lambda^{4}}$ & - & - \\
$C_{\phi t b}$ & $\ldots$ & $\ldots$ & $\ldots$ & $\ldots$ & $\ldots$ & $\frac{v^{4}}{\Lambda^{4}}$ & $\frac{m_{t} v^{3}}{\Lambda^{4}} \frac{m_{W}^{2}}{s} \ln \frac{s}{m_{W}^{2}}$ \\
$C_{b W}$ & $\ldots$ & $\ldots$ & $\ldots$ & $\ldots$ & $\ldots$ & $\ldots$ & $\frac{v^{2} m_{W}^{2}}{\Lambda^{4}}$ \\
\hline
\end{tabular}

Table 4. Relative scaling of operator contributions in $t$-channel single top production at high energies $\sqrt{s} \gg m_{t}$. The scaling for $s$-channel production is obtained by replacing $m_{W}^{2} \rightarrow s$ and $\ln (\ldots) \rightarrow 1$. The SM contribution scales as $1 / m_{W}^{2}$ in $t$-channel production and as $1 / s$ in $s$-channel production. We denote a negligible bottom mass insertion as $\propto m_{b}$.

The operator $O_{\phi Q}^{3}$ has the same Lorentz structure as the $t b W$ coupling in weak interactions, so its interference with the SM causes a constant shift in the rate. In turn, the contributions of $O_{Q q}^{3,1}$ is logarithmically enhanced at high energies, while $O_{t W}$ scales as $\ln \left(s / m_{W}^{2}\right) / s$. In $s$-channel production the logarithmic enhancement is absent. In table 4 , we summarize the relative scaling of operators with respect to the SM for $t$ - and $s$-channel production.

Once we include dimension-6 squared terms, three additional operators $O_{Q q}^{3,8}, O_{\phi t b}$ and $O_{b W}$ contribute to single top production. The operator $O_{Q q}^{3,8}$ does not interfere because of its color structure. The interference of $O_{\phi t b}$ and $O_{b W}$ with the SM and all other operators is suppressed by the bottom mass $m_{b}$, because $O_{\phi t b}$ and $O_{b W}$ involve right-handed bottom quarks. This means that their interference with left-handed currents is helicity-suppressed. The interference between $O_{\phi t b}$ and $O_{b W}$ are not $m_{b}$-suppressed and thus much larger than their interference with the SM amplitude. 
Top decay is sensitive to the operators $O_{Q q}^{3,1}$ (or operators with two heavy quarks and two leptons in semi-leptonic top decays), $O_{\phi Q}^{3}$ and $O_{t W}$ in SM-interference. Since the $W$ boson in $t \rightarrow b W \rightarrow b q \bar{q}^{\prime}$ decays is on-shell in the observables we consider, the contribution of four-quark operators is negligible. As in single top production, $O_{\phi Q}^{3}$ re-scales the SM rate by a factor $\left(1+2 C_{\phi Q}^{3} v^{2} / \Lambda^{2}\right)$. With the current experimental situation, we expect a higher sensitivity to $O_{\phi Q}^{3}$ in $t$-channel single top production than in the top width $\Gamma_{t}$. The dipole operator $O_{t W}$ in turn modifies the top decay kinematics. In particular, the helicity fractions $F_{i}=\Gamma_{i} / \Gamma_{t}$ of the $W$-boson are very sensitive to this operator. In our analysis, we consider [16]

$$
\begin{aligned}
& F_{L}=\frac{m_{t}^{2}}{m_{t}^{2}+2 m_{W}^{2}}-4 \sqrt{2} \frac{v^{2}}{\Lambda^{2}} C_{t W} \frac{m_{t} m_{W}\left(m_{t}^{2}-m_{W}^{2}\right)}{\left(m_{t}^{2}+2 m_{W}^{2}\right)^{2}} \\
& F_{0}=\frac{m_{t}^{2}}{m_{t}^{2}+2 m_{W}^{2}}+4 \sqrt{2} \frac{v^{2}}{\Lambda^{2}} C_{t W} \frac{m_{t} m_{W}\left(m_{t}^{2}-m_{W}^{2}\right)}{\left(m_{t}^{2}+2 m_{W}^{2}\right)^{2}}
\end{aligned}
$$

where $F_{L}$ and $F_{0}$ denote the $t \rightarrow b W$ branching ratios into $W$ bosons with negative $(L)$ and zero (0) helicity, respectively. At the level of dimension- 6 contributions squared, $O_{b W}$ and $O_{\phi t b}$ contribute to the helicity fractions. Numerically their contribution is at the permille level, similar to $t$-channel production. In a global analysis, top decays are thus relevant in probing these operators.

Associated $\boldsymbol{t} \boldsymbol{W}$ production probes $O_{\phi Q}^{3}, O_{t W}$ and $O_{t G}$ interfering with the SM amplitudes. Obviously, its sensitivity to $O_{t G}$ is much smaller than for top pair production. The operator $O_{\phi Q}^{3}$ is also probed in $t$-channel and $s$-channel production, and $O_{t W}$ is best probed in top decays. We therefore do not expect much additional information on SMEFT operators from $t W$ production.

Associated $t Z$ production is essentially $t$-channel single top production with an additional $Z$-boson in the final state. It probes all operators that contribute to $t$-channel production, as shown in table 1 . The contribution of an operator relative to the SM, however, is different for the two processes. In general, $t Z$ production probes operators at higher energies than $t$-channel production, leading to enhanced effects of operators that grow with energy [49]. A larger theoretical sensitivity in $t Z$ production can thus compensate for the lower experimental sensitivity.

In addition, $t Z$ production probes operators that modify the top coupling to the $Z$ boson, namely $O_{\phi Q}^{-}, O_{\phi t}$, and $O_{t Z}$. All three operators interfere with the SM. Their scaling at high energies depends on the polarization of the $Z$-boson and has been studied in detail in refs. [5, 49]. The operators $O_{\phi Q}^{-}$and $O_{\phi t}$ modify the SM $Z$ couplings with left- and right-handed tops, respectively. The main difference is observed in the longitudinal $Z$ mode. While the $O_{\phi Q^{-}}^{-} \mathrm{SM}$ interference grows with energy, the $O_{\phi t^{-}} \mathrm{SM}$ interference requires a helicity flip of the top quark and thus does not feature this growth. Similar considerations apply at the level of $O_{\phi Q}^{-}-O_{\phi Q}^{-}$and $O_{\phi t}-O_{\phi t}$ interference. We therefore expect a higher sensitivity to $O_{\phi Q}^{-}$than to $O_{\phi t}$, which we will confirm numerically in section 5 . The dipole operator $O_{t Z}$ features a similar energy growth in longitudinal $Z$-production as $O_{\phi Q}^{-}$, resulting in a sizeable contribution to $t Z$ production. 
Associated single top production with a Higgs boson is an interesting channel that complements $t W$ and $t Z$ production [49]. In addition to the gauge operators $t H$ production probes modifications of the top Yukawa coupling. We do not consider $t H$ production in our fit here, but plan to include it in a combined analysis with Higgs observables.

NLO QCD corrections can modify the kinematics of operator contributions in single top production. However, the number of single top observables is large enough to distinguish between all contributing operators already at leading order. We therefore do not investigate NLO contributions in detail here, but include them in our numerical analysis. For $t$-channel single top production and top decay, NLO corrections in SMEFT have been calculated in refs. [50, 51].

\subsection{Associated $t \bar{t} W$ and $t \bar{t} Z$ production}

Compared with top pair and single top production, associated $t \bar{t} W$ and $t \bar{t} Z$ production do not bring us sensitivity to new operators, but probe them in a different context. The main purpose of including these two processes in our global analysis is to resolve blind directions and to better probe operators that are difficult to access in other channels. Just as for the $t H$ production channel we leave $t \bar{t} H$ production [52] to a future combination with global Higgs analyses.

Associated $t \bar{t} W$ production is sensitive to a subset of the possible four-quark operators. Since the $W$ can only be radiated from the initial state in the SM, only operators with lefthanded initial quarks contribute while $R R$ and $L R$ contributions are absent if we neglect light-quark masses. The non-trivial electroweak structure of $t \bar{t} W$ production affects the relative contributions of the weak singlet and triplet operators $O_{Q q}^{1,8}$ and $O_{Q q}^{3,8}$. We will use this effect to distinguish between these two operators. In the SM, the total rate of $t \bar{t} W$ production is dominated by quark-antiquark interactions, while inclusive $t \bar{t}$ production is gluon-dominated. This means that relative to the SM contribution four-quark operators thus give sizeable effects in the $t \bar{t} W$ rate and we can hope for a good sensitivity to $L L$ and $R L$ operators.

Associated $t \bar{t} Z$ probes the same four-quark operators that enter $t \bar{t}$ production. Similarly to $t \bar{t} W$ production, the emission of the boson changes the relative contributions of four-quark operators with different weak gauge structure. In addition, $t \bar{t} Z$ is sensitive to operators with right-handed up versus down quarks, namely $O_{t u}^{8}$ and $O_{t d}^{8}$ or $O_{Q u}^{8}$ and $O_{Q d}^{8}$. In section 4.2 , we will disentangle these operators by combining $t \bar{t}$ distributions with $t \bar{t} W$ and $t \bar{t} Z$ production in a global analysis. In addition, we use the $t \bar{t} Z$ process to probe $O_{\phi Q}^{-}, O_{\phi t}$ and $O_{t Z}$, which are so far constrained by $t Z$ production. In $t \bar{t} Z$ production, all three operators interfere with the SM. The contributions of $O_{\phi Q}^{-}$and $O_{\phi t}$ re-scale the SM $Z$-couplings to left- and right-handed tops, but do not change the process kinematics. The dipole operator $O_{t Z}$ changes the kinematic distributions mildly, but its overall effect on the rate is modest [53]. Combining searches for $O_{t A}$ in $t \bar{t} \gamma$ and $O_{t W}$ in top decays is an alternative way to get access to $C_{t Z}$, see eq. (2.6) and ref. [54]. 


\begin{tabular}{|c|c|c|c|c|c|c|c|c|c|c|}
\hline experiment & $\sqrt{S}(\mathrm{TeV})$ & $\mathcal{L}\left(\mathrm{fb}^{-1}\right)$ & channel & observable & $K$-factor & \#bins & $\mathrm{R}$ & $\mathrm{M}$ & $\mathrm{D}$ & A \\
\hline \multicolumn{11}{|l|}{$p p \rightarrow t \bar{t}$} \\
\hline CMS [55] & 8 & 19.7 & $e \mu$ & $\sigma_{t \bar{t}}$ & {$[56]$} & & $\checkmark$ & $\checkmark$ & . & \\
\hline ATLAS [57] & 8 & 20.02 & $l j$ & $\sigma_{t \bar{t}}$ & {$[56]$} & & $\checkmark$ & $\checkmark$ & . & \\
\hline CMS $\quad[58]$ & 13 & 2.3 & $l j$ & $\sigma_{t \bar{t}}$ & {$[56]$} & & $\checkmark$ & $\checkmark$ & . & \\
\hline CMS [59] & 13 & 3.2 & $l l$ & $\sigma_{t \bar{t}}$ & {$[56]$} & & $\checkmark$ & $\checkmark$ & . & \\
\hline ATLAS [60] & 13 & 3.2 & $e \mu$ & $\sigma_{t \bar{t}}$ & {$[56]$} & & $\checkmark$ & $\checkmark$ & . & . \\
\hline ATLAS [61] & 8 & 20.3 & $l j$ & $\sigma^{-1}\left(d \sigma / d m_{t \bar{t}}\right)$ & {$[62-64]$} & 7 & . & $\checkmark$ & $\checkmark$ & \\
\hline \multirow[t]{2}{*}{ CMS [65] } & 8 & 19.7 & $l j$ & $\sigma^{-1}\left(d \sigma / d p_{T, t}\right)$ & {$[62-64]$} & 7 & . & . & $\checkmark$ & . \\
\hline & & & $l l$ & $\sigma^{-1}\left(d \sigma / d p_{T, 1}\right)$ & & 5 & . & . & $\checkmark$ & \\
\hline CMS [66] & 8 & 19.7 & $e \mu$ & \multicolumn{2}{|c|}{$\sigma^{-1}\left(d^{2} \sigma / d m_{t \bar{t}} d y_{t \bar{t}}\right)[67]^{a}$} & 16 & . & . & . & . \\
\hline CMS $\quad[68]$ & 8 & 19.7 & $l j$ high $p_{T}$ & \multicolumn{2}{|c|}{$d \sigma / d p_{T, t}$} & 5 & . & . & . & \\
\hline CMS [69] & 13 & 2.3 & $l j$ & \multicolumn{2}{|l|}{$\sigma^{-1}\left(d \sigma / d m_{t \bar{t}}\right)$} & 8 & . & $\checkmark$ & $\checkmark$ & \\
\hline CMS [70] & 13 & 35.8 & $l j$ & \multicolumn{2}{|c|}{$\sigma^{-1}\left(d \sigma / d p_{T}\left(t_{h}\right)\right)[62-64]$} & 12 & . & . & $\checkmark$ & . \\
\hline CMS [71] & 13 & 2.1 & $l l$ & \multicolumn{2}{|c|}{$\sigma^{-1}\left(d \sigma / d p_{T, t}\right) \quad[62-64]$} & 6 & . & . & $\checkmark$ & . \\
\hline CMS $\quad[72]$ & 13 & 35.9 & $l l$ & $\sigma^{-1}\left(d \sigma / d \Delta y_{t \bar{t}}\right)$ & {$[62-64]$} & 8 & . & . & . & $\checkmark$ \\
\hline ATLAS [73] & 13 & 36.1 & aj high $p_{T}$ & \multicolumn{2}{|l|}{$\sigma^{-1}\left(d \sigma / d m_{t \bar{t}}\right)$} & 8 & . & . & . & . \\
\hline CMS $[74]$ & 8 & 19.7 & $l j$ & $A_{C}$ & {$[75]$} & & . & . & . & $\checkmark$ \\
\hline CMS $\quad[76]$ & 8 & 19.7 & $l l$ & $A_{C}$ & {$[75]$} & & . & . & . & $\checkmark$ \\
\hline ATLAS [77] & 8 & 20.3 & $l j$ & $A_{C}$ & {$[75]$} & & . & . & . & $\checkmark$ \\
\hline ATLAS [78] & 8 & 20.3 & $l l$ & $A_{C}$ & {$[75]$} & & . & . & . & $\checkmark$ \\
\hline ATLAS [79] & 13 & 139 & $l j$ & $A_{C}$ & {$[75]$} & & . & . & . & $\checkmark$ \\
\hline \multicolumn{11}{|l|}{$p p \rightarrow t \bar{t} Z$} \\
\hline CMS [80] & 13 & 77.5 & multi lept. & $\sigma_{t \bar{t} Z}$ & {$[81]$} & & . & . & . & . \\
\hline ATLAS [82] & 13 & 3.2 & multi lept. & $\sigma_{t \bar{t} Z}$ & [81] & & . & . & . & . \\
\hline \multicolumn{11}{|l|}{$p p \rightarrow t \bar{t} W$} \\
\hline CMS [83] & 13 & 35.9 & multi lept. & $\sigma_{t \bar{t} W}$ & {$[81]$} & & . & . & . & \\
\hline ATLAS [82] & 13 & 3.2 & multi lept. & $\sigma_{t \bar{t} W}$ & {$[81]$} & & . & . & . & \\
\hline
\end{tabular}

${ }^{a}$ Tables available at www.precision.hep.phy.cam.ac.uk/results/ttbar-fastnlo/.

Table 5. Top-pair observables included in our global analysis. The labels R, M, D, A define four different data sets with rates, rates and invariant mass distributions, distributions only, and asymmetries, used in the numerical analysis of section 4 .

\section{Global analysis setup}

\subsection{Data set}

The key to any global analysis is the availability of enough measurements to constrain the model parameters. In case of the top sector we confront 22 dimension- 6 operators with a much larger number of available measurements shown in tables 5 and 6 . The data forms two main parts, measurements of the leading top pair production process mediated 


\begin{tabular}{|c|c|c|c|c|c|}
\hline experiment & $\sqrt{S}(\mathrm{TeV})$ & $\mathcal{L}\left(\mathrm{fb}^{-1}\right)$ & channel & observable & $K$-factor \\
\hline \multicolumn{6}{|l|}{ t-channel } \\
\hline CMS [84] & 7 & $1.17(\mu), 1.56(e)$ & $e+\mu$ & $\sigma_{t q+\bar{t} q}$ & \\
\hline ATLAS [85] & 7 & 4.59 & $e+\mu$ & $\sigma_{t q+\bar{t} q}$ & \\
\hline ATLAS [86] & 8 & 20.2 & $e+\mu$ & $\sigma_{t q}, \sigma_{\bar{t} q}$ & \\
\hline CMS [87] & 8 & 19.7 & $e+\mu$ & $\sigma_{t q}, \sigma_{\bar{t} q}$ & \\
\hline ATLAS [88] & 13 & 3.2 & $e+\mu$ & $\sigma_{t q}, \sigma_{\bar{t} q}$ & [89] \\
\hline CMS [90] & 13 & 2.3 & $\mu$ & $\sigma_{t q}, \sigma_{\bar{t} q}$ & [89] \\
\hline \multicolumn{6}{|l|}{$s$-channel } \\
\hline \multirow[t]{2}{*}{ CMS } & 7 & 5.1 & $\mu$ & $\sigma_{t \bar{b}+\bar{t} b}$ & \\
\hline & 8 & 19.7 & $e+\mu$ & $\sigma_{t \bar{b}+\bar{t} b}$ & \\
\hline ATLAS [92] & 8 & 20.3 & $e+\mu$ & $\sigma_{t \bar{b}+\bar{t} b}$ & \\
\hline \multicolumn{6}{|l|}{$t W$ channel } \\
\hline ATLAS [93] & 7 & 2.05 & $2 l j$ & $\sigma_{t W+\bar{t} W}$ & \\
\hline CMS [94] & 7 & 4.9 & $2 l j$ & $\sigma_{t W+\bar{t} W}$ & \\
\hline ATLAS [95] & 8 & 20.3 & $2 l j$ & $\sigma_{t W+\bar{t} W}$ & \\
\hline CMS $\quad[96]$ & 8 & 12.2 & $2 l j$ & $\sigma_{t W+\bar{t} W}$ & \\
\hline ATLAS [97] & 13 & 3.2 & $2 l j$ & $\sigma_{t W+\bar{t} W}$ & \\
\hline CMS $\quad[98]$ & 13 & 35.9 & $e \mu j$ & $\sigma_{t W+\bar{t} W}$ & \\
\hline \multicolumn{6}{|l|}{$t Z$ channel } \\
\hline ATLAS [99] & 13 & 36.1 & $3 l 2 j$ & $\sigma_{t Z q}$ & \\
\hline \multicolumn{6}{|c|}{$W$ helicities in top decays } \\
\hline ATLAS [100] & 7 & 1.04 & & $F_{0}, F_{L}$ & \\
\hline CMS [101] & 13 & 5.0 & & $F_{0}, F_{L}$ & \\
\hline ATLAS [102] & 8 & 20.2 & & $F_{0}, F_{L}$ & \\
\hline CMS & 8 & 19.8 & & $F_{0}, F_{L}$ & \\
\hline
\end{tabular}

Table 6. Observables included in the single top fit, in analogy to table 5.

by QCD couplings and measurements of processes including a weak coupling. The latter include single top production as well as associated top pair production with electroweak bosons. Because all our measurements are unfolded to the level of stable top quarks, and because there is essentially only one top decay channel leading to a universal branching ratio of one, we can assume SM-like top decays for all measurements except for the $W$ helicity fractions in top decays. Observables combining top production and decay play a special role in the SMEFT interpretation, because they probe features of operators not accessible in top production alone [34, 104-106]. In our analysis, the charge asymmetry described in section 2.1 plays a similar role in probing operators, even though it is based on kinematics of fully reconstructed top quarks. 
In terms of the Wilson coefficients of section 2 all our rate observables have the form

$$
\sigma=\sigma_{\mathrm{SM}}+\sum_{k} \frac{C_{k}}{\Lambda^{2}} \sigma_{k}+\sum_{k, l} \frac{C_{k} C_{l}}{\Lambda^{4}} \sigma_{k l},
$$

where $\sigma_{\mathrm{SM}}$ is the SM prediction, $\sigma_{k}$ are contributions arising from the interference of a single dimension- 6 operator with the SM, and $\sigma_{k l}$ arise from the interference of two diagrams containing one operator each. Technically, $\sigma_{k}$ and $\sigma_{k l}$ are the theory input which MADGRAPH5_AMC@NLO [36] provides at NLO QCD accuracy.

The SMEFT Lagrangian leads to two main kinds of corrections, as illustrated for top pair production in table 2: operators which change the high-energy behavior of the process through an additional energy dependence of the kind $s / \Lambda^{2}$ and those which scale merely like $v^{2} / \Lambda^{2}$ or $m_{t} v / \Lambda^{2} \approx y_{t} v^{2} / \Lambda^{2}$ compared to the SM. For the latter the leading observables are rate measurements or the total cross section, because they offer the best statistics and often minimize theoretical uncertainties. From global Higgs-electroweak analyses we know that a modified momentum dependence can be constrained most efficiently by high-energy tails of kinematic distributions or simplified template cross sections $[8,10-14] .^{3}$ Similarly, we know that for many kinematic distributions the few bins with the highest momentum transfer include the relevant information on individual operators, whereas for several operators with a different high-energy behavior there often exists several relevant regimes [107].

Unlike in the Higgs sector, cross section measurements in the top sector are reported such that we can easily compare them to parton-level predictions. Kinematic distributions are typically reported as normalized distributions, i.e. they integrate to one and can be combined with total rate measurements without double-counting information. A problem arises when we include operator contributions to the distribution in the numerator and to the rate in the denominator. In this case the normalized bin entries entering our fit become correlated and develop a distinct non-linear behavior.

\subsection{SFitter analysis}

For our global LHC analysis we use the SFITTER framework [38, 39], which focuses on a proper treatment of uncertainties in a conservative frequentist approach. We extract the statistical uncertainties and a leading set of up to $\sim 20$ systematic uncertainties for each experiment and simulate a Gaussian shape of the completely exclusive likelihood for statistics and systematics. For the systematic uncertainties we also allow for correlations within the same experiment, collider energy scale, and top signature. This applies for example to jet uncertainties like the jet energy scale or the jet efficiencies. An exception is the uncertainty on the luminosity, which we correlate for all channels and both experiments. In order to simplify the treatment of uncertainty correlations, we fit only one observable from each experimental analysis, and we never take two measurements of the same observable at the same energy scale. Moreover, for total rates and charge asymmetries we fit only two observables, one for each collider energy, obtained with weighted averages of the measurements performed with different experiments and datasets.

\footnotetext{
${ }^{3}$ Note that for a distribution to constrain a dimension- 6 contribution in this phase space region it is not necessary that we actually observe the SM process in the same phase space region [13].
} 
In addition to the experimental sources of uncertainties, theoretical error bars reflect missing higher orders in the perturbative series. Precise predictions are crucial to extract any Lagrangian parameter from LHC rate measurements. We rely on NLO QCD predictions for $t \bar{t}$ and single top observables in SMEFT using MADGRAPH5_AMC@NLO, while we use LO QCD predictions for the statistics-limited $t \bar{t} V$ rates. For the central values of cross sections and bins in differential distributions, we add $K$-factors to include NNLO QCD corrections in the SM, whenever available (see tables 5,6). This means we assume that the operator contributions scale like the SM rate beyond NLO. The only exception is the charge asymmetry, that does not scale multiplicatively with higher-order corrections. In this case we fit the sum of the most precise available SM prediction and the new physics corrections at (N)LO from MADGraPH5_AMC@NLO. Electroweak corrections and resummation effects are not included in our predictions, as they are well within the assumed theoretical uncertainties. We retain only diagrams with on-shell tops, as off-shell effects generally amount to a few percent or less for the measurements considered here, see e.g. [108-110] for related SM calculations. EFT corrections to off-shell diagrams are expected to be analogously suppressed and therefore irrelevant in our fit, given the current sensitivity. EFT contributions to top quark decays are neglected in $t \bar{t}(V)$ production, but constrained independently in measurements of the $W$ helicity fractions, which are expected to have a significantly stronger sensitivity.

The theoretical uncertainties are obtained by varying the renormalization and factorization scales by a factor of two around the respective central scale choices. Since technically we cannot distinguish the uncertainties due to operator effects, we use the scale uncertainties on the SM prediction from our NLO simulations as an overall theory uncertainty on the observable. This gives for instance a $12 \%$ uncertainty for the combined $\mathrm{SM}$ and dimension- $6 t \bar{t}$ and $t \bar{t} V$ rates. Regarding higher-order corrections, our estimate based on the NLO scale dependence in the presence of dimension- 6 effects is likely to be conservative for small new physics effects [111]. Since NLO corrections to the non-SM contributions are included in our simulations, we generally expect QCD effects beyond NLO to be moderate. Exceptions occur in single top production, where SM QCD effects first occur beyond tree level, or in bins of kinematic distributions near the endpoints of the spectrum. To be conservative, whenever the scale uncertainty in the NLO simulation for such rate measurements happens to be very small we replace it by a minimum of $10 \%$. Similarly, when strong cancellations of scale uncertainties occur in normalized kinematic distributions, we replace the theoretical uncertainty in each bin by $2 \%$ whenever the scale variation drops below this level $[112,113]$.

In SFitTer all theoretical uncertainties are modelled as a flat likelihood within the quoted error band. This applies to the theoretical uncertainties on the signal as well as the theoretical uncertainties on the background, quoted in the experimental analyses. If we combine them with Gaussian experimental uncertainties in a profile likelihood this leads to the RFiT scheme [114]. Theoretical uncertainties are generally uncorrelated unless they describe the same fiducial volume at the same collider energy. This also includes the theoretical errors for individual bins in a kinematic distribution, which we assume to be uncorrelated. Uncertainties from the limited precision of parton densities are evaluated 
in analogy to the theoretical uncertainties reflecting the missing higher orders in the hard process. We evaluate them using a set of 209 predictions from the NNPDF3.0 NLO set with $\alpha_{s}\left(m_{Z}\right)=0.118$ [115], the Mмнт2014NLO set at 68\% CL [116], and the CT14NLO set [117]. A typical error bar from the parton distribution functions (PDFs) is $6 \%$ for the $t \bar{t}$ or $t \bar{t} V$ rates. Because we assume a flat likelihood for these PDF uncertainties, the profile likelihood combination of higher-order and PDF uncertainties adds the two error bars linearly.

To probe the parameter space we rely on Markov chains, similar to ref. [8], rather than the numerically more complex toy measurements used in ref. [13]. To cover the full 22-dimensional parameter space we use up to 2000 Markov chains giving up to 400 million parameter points. This defines our fully exclusive likelihood which we then profile down to two and one relevant dimensions.

\section{Top pair features}

Before entering a global analysis of the top sector we study some of the underlying features in detail. This is essential for the top-pair side of the analysis. Its unique challenges are very different from the electroweak-Higgs sector $[8,11-13]$ and the single top sector discussed in section 5 .

In top pair production the operator $O_{t G}$ induces large corrections to the total and differential rates, as it is the only operator modifying the gluon-induced production process. We discuss its known and expected behavior in section 4.1 and roughly estimate the expected sensitivity of our global fit.

The new feature in top pair production is the large set of four-quark operators affecting the partonic process $q \bar{q} \rightarrow t \bar{t}$. Fourteen such operators, different in their QCD and electroweak structure, contribute to one and the same process. Since top pair production is a QCD process, most of its observables average or sum over the electroweak properties of the external particles. To distinguish these operators we rely on the observables

$$
\left\{\sigma_{\text {tot }}, \frac{d \sigma}{d m_{t \bar{t}}}, \frac{d \sigma}{d p_{T, t}}, \frac{d \sigma}{d \Delta y_{t \bar{t}}}, A_{C}\right\}
$$

supplemented by $p_{T, t}$ and $m_{t \bar{t}}$ distributions in the boosted region. In sections 4.2 to 4.4 we will study how the gauge and chiral structure of four-quark operators can be resolved by dedicated $t \bar{t}$ measurements. This allows us to break some of the flat directions in model space already at LO in the EFT analysis, where only the tree-level interference between the SM and the dimension-6 operators are considered.

Finally, in section 4.5 we will study the effect of dimension-6-squared contributions on the sensitivity to operators. We will see that the flat directions turn into compact circles which allow us to derive more stringent limits on individual operators.

\subsection{Event kinematics}

Before we study the effects of specific dimension-6 operators on top pair production, we roughly estimate the reach of our analysis for operators affecting the total rate and for 
operators affecting the event kinematics. The top-gluon dipole operator $O_{t G}$ is the only top EFT contribution to the leading partonic process $g g \rightarrow t \bar{t}$. We therefore expect a high sensitivity to $O_{t G}$ in inclusive top pair production. In contrast, four-quark operators contribute only to the $q \bar{q} \rightarrow t \bar{t}$ process, which is subleading, but enhanced at high energies. We thus expect the best sensitivity to four-quark operators in tails of kinematic distributions.

In our analysis, all distributions are normalized to the total rate. These normalized distributions are direct probes of the dynamics of operator contributions relative to the SM. To illustrate this important point, we compare the event kinematics of the dipole operator $O_{t G}$ with the four-quark operator $O_{t u}^{8}$. The normalized $m_{t \bar{t}}$ distribution depends on these two operators as (neglecting $O_{t G}-O_{t u}^{8}$ interference)

$$
\begin{aligned}
\frac{1}{\sigma} \frac{d \sigma}{d m_{t \bar{t}}} \approx \frac{\sigma_{\mathrm{SM}}\left(m_{t \bar{t}}\right)}{\sigma_{\mathrm{SM}}\left(2 m_{t}\right)}(1 & +\mathcal{O}\left(m_{t} v-m_{t} v\right) \frac{C_{t G}}{\Lambda^{2}}+\mathcal{O}\left(m_{t \bar{t}}^{2}-\left(2 m_{t}\right)^{2}\right) v^{2} \frac{\left|C_{t G}\right|^{2}}{\Lambda^{4}} \\
& \left.+\mathcal{O}\left(m_{t \bar{t}}^{2}-\left(2 m_{t}\right)^{2}\right) \frac{C_{t u}^{8}}{\Lambda^{2}}+\mathcal{O}\left(m_{t \bar{t}}^{4}-\left(2 m_{t}\right)^{4}\right) \frac{\left|C_{t u}^{8}\right|^{2}}{\Lambda^{4}}\right) .
\end{aligned}
$$

Here $2 m_{t}$ denotes the invariant mass close to the production threshold, which dominates in the total cross section, while $m_{t \bar{t}}$ can be much higher in differential distributions. Since the $O_{t G^{-}}$QCD interference does not feature an energy enhancement, it cancels almost completely in normalized distributions. Kinematic distributions are therefore expected to lead to relatively weak constraints driven by the $\left|C_{t G}\right|^{2}$ term. In contrast, the four-quark contribution of $O_{t u}^{8}$ features an energy enhancement already at $\mathcal{O}\left(\Lambda^{-2}\right)$. This leads to a good sensitivity at high energies, despite the relative suppression by the parton luminosity. Total rates and distributions are thus complementary in probing dipole operators and four-quark operators. Notice that in our numerical analysis we keep the full operator contributions in the normalization of distributions.

The reach of measurements of total cross sections at $8 \mathrm{TeV}$ and $13 \mathrm{TeV}$ and a $p_{T}$ distribution at $13 \mathrm{TeV}$ is estimated for $O_{t G}$ and $O_{t u}^{8}$ in figure 3 .

The upper-left panel shows the averaged cross section measurements with their combined uncertainties. Similarly, the upper-right panel shows the normalized $p_{T}$ distribution for the hadronically decaying top at $13 \mathrm{TeV}$ from ref. [70] (see table 5). The two lower panels show the relative deviations from the SM prediction and the $68 \%$ CL limits from a combined analysis of $C_{t G}$ and $C_{t u}^{8}$ to the small data set consisting of only the observables shown in figure 3. The grey panels show the result from the new physics interference at order $\Lambda^{-2}$ for $C_{t G}$ in terms of the total rates and for $C_{t u}^{8}$ in terms of the kinematic distribution, corresponding to the $68 \%$ CL ranges

$$
C_{t G} / \Lambda^{2} \in[-0.19,0.78] / \mathrm{TeV}^{2} \quad C_{t u}^{8} / \Lambda^{2} \in[-6.77,-0.57] / \mathrm{TeV}^{2} .
$$

The limits are slightly asymmetric, because the top quarks in the normalized distribution are softer than in the SM expectation.

For the red $\left(O_{t G}\right)$ and blue $\left(O_{t u}^{8}\right)$ shaded regions we also include the contributions to order $\Lambda^{-4}$ and find at $68 \% \mathrm{CL}$

$$
C_{t G} / \Lambda^{2} \in[-0.07,0.58] / \mathrm{TeV}^{2} \quad C_{t u}^{8} / \Lambda^{2} \in[-1.72,0.26] / \mathrm{TeV}^{2} .
$$



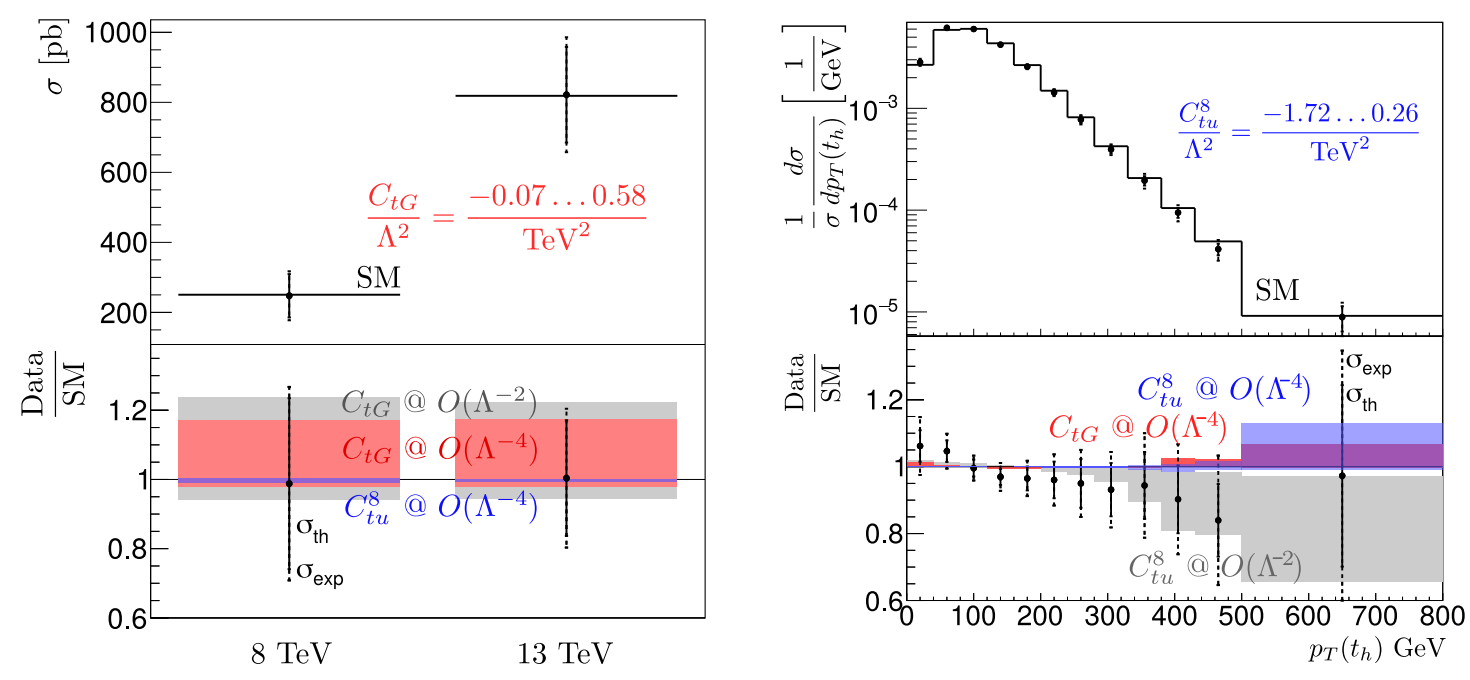

Figure 3. Contribution of $O_{t G}$ and $O_{t u}^{8}$ to total $t \bar{t}$ rates (left) and the normalized $p_{T}$ distribution of a hadronically decaying top (right). The shaded regions correspond to the $68 \% \mathrm{CL}$ from a simultaneous fit to the two rates and the distribution. The grey shaded regions show the contribution from $O_{t G}$ (rates) and $O_{t u}^{8}$ (distribution) at order $\Lambda^{-2}$. The red and blue shaded regions show the contribution from $O_{t G}$ and $O_{t u}^{8}$ to order $\Lambda^{-4}$, respectively.

While these limits are just based on a small fit to three observables, they give us an intuition of what to expect from our fit. For $C_{t G}$ an expected range around $\Lambda / \sqrt{\left|C_{t G}\right|}=1.3 \mathrm{TeV}$ saturates the error bars of the leading total rate measurement, while for the four-quark operator $C_{t u}^{8}$ values around $\Lambda / \sqrt{\left|C_{t u}^{8}\right|}=0.7 \mathrm{TeV}$ can be expected from this one kinematic distribution. Comparing these limits to the kinematic range probed by the $p_{T}$ distribution in figure 3, we see that the effective theory interpretation is valid for an underlying theory that does not predict propagating new states at the LHC and is not too strongly coupled.

In figure 4 we show how total rates and normalized kinematic distributions lead to very different likelihood distributions. First, rate measurements alone have a strong constraining power on $C_{t G}$ compared to four-quark operators, due to the SMEFT correction being relatively large. The likelihood from normalized distribution is strongly asymmetric: negative values of $C_{t G}$ are strongly limited by the physical requirement that the bin content of all the measured distributions remains positive. Positive values of $C_{t G}$, on the other hand, are less constrained as discussed in the previous section. More specifically, let $n_{k}$ be the number of entries in $k$-th bin of a normalized distribution. As a function of the $\mathrm{SM}\left(n_{k}^{S M}\right)$ and $\mathrm{SM}-C_{t G}$ interference $\left(n_{k}^{\text {int }}\right)$ contributions, it scales as

$$
\frac{n_{k}^{\mathrm{SM}}+n_{k}^{\mathrm{int}} C_{t G} / \Lambda^{2}}{\sum_{l}\left(n_{l}^{\mathrm{SM}}+n_{l}^{\mathrm{int}} C_{t G} / \Lambda^{2}\right)} \stackrel{C_{t G} \rightarrow \infty}{\longrightarrow} \frac{n_{k}^{\mathrm{int}}}{\sum_{l} n_{l}^{\mathrm{int}}} .
$$

For large values of $C_{t G} / \Lambda^{2} \rightarrow \infty$ the normalized bin content becomes a constant. As noted in eq. (4.2), $C_{t G}$ is characterized by a kinematic behavior very similar to that of the SM, which leads to values $n_{k}^{\text {int }} / \sum_{l} n_{l}^{\text {int }}$ generally compatible with $n_{k}^{\mathrm{SM}} / \sum_{l} n_{l}^{\mathrm{SM}}$. As a consequence the corresponding log-likelihood also converges to constant $>0$. This asymptotic behavior is not observed once quadratic terms are included, because the sensitivity to $C_{t G}$ 

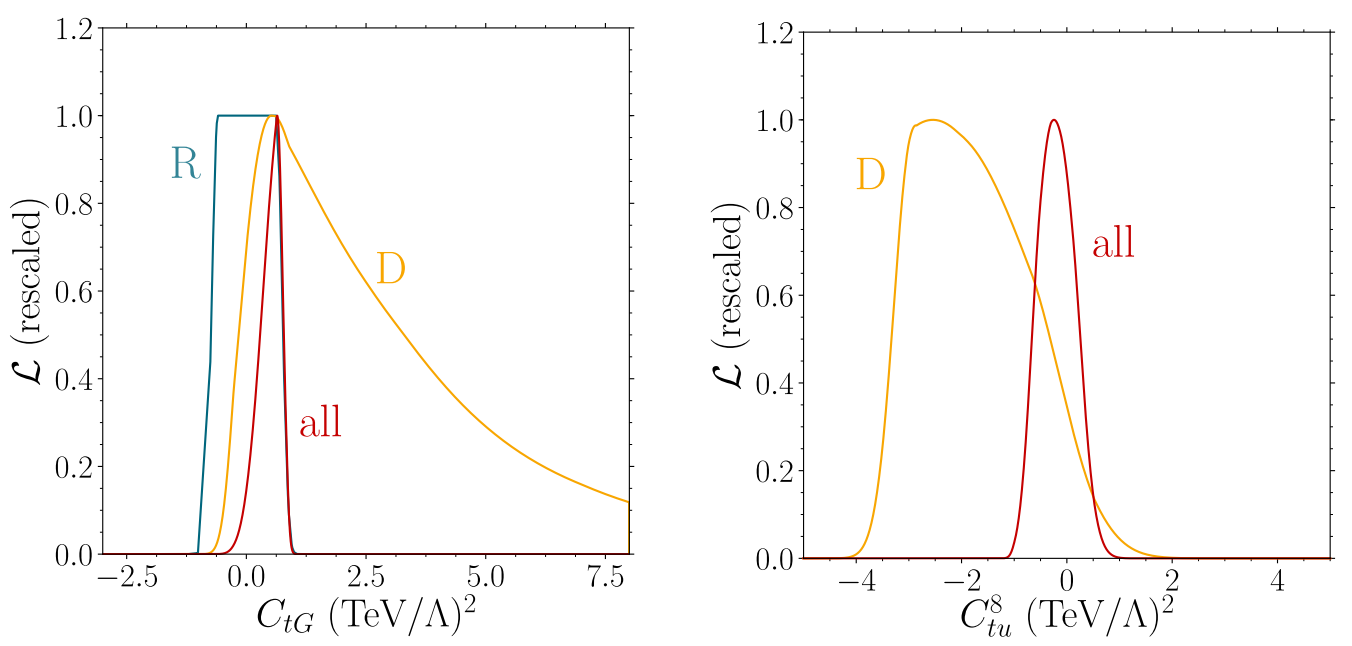

Figure 4. Normalized likelihood as a function of $C_{t G}$ (left) and a $C_{t u}^{8}$ (right) in individual fits at LO to order $\Lambda^{-2}$. We show fits to the R (blue) and D (yellow) observable sets of table 5 , and to all $t \bar{t}$ observables in table 5 (red). In the right panel, we do not show the $\mathrm{R}$ likelihood, as it is 1 for all the values of $C_{t u}^{8}$ in the displayed range.

is enhanced by $s v^{2} / \Lambda^{4}$ in high-energy bins, see eq. (4.2) and table 2. Combining all $t \bar{t}$ measurements the likelihood recovers a fairly symmetric form, but with a distinct shift of the minimum towards small positive values of $C_{t G}$.

For comparison, the asymptotic behavior in the linear fit is not observed for an interfering four-quark operator like $C_{t u}^{8}$, because it induces a significantly different shape in the kinematic distributions compared to the SM, scaling as $s / \Lambda^{2}$. Large values of the Wilson coefficients are therefore strongly disfavored by at least one of the bin measurements that drive the log-likelikood towards zero. In the right panel of figure 4, we show that $C_{t u}^{8}$ is well constrained by measurements of normalized distributions. Total rates have little impact on the fit results.

\subsection{Incoming up versus down quarks}

The set of four-quark operators laid out in eq. (2.3) and eq. (2.4) span all possible assignments of the quark fields to representations the SM symmetry groups:

1. chirality of the light quark and top quark currents;

2. left-handed currents: singlet or triplet under $\mathrm{SU}(2)_{L}$;

3. right-handed currents: up- or down-type light quarks;

4. singlet or octet color contraction of the currents.

Top pair production through strong interactions is not sensitive at parton level to the nature of the incoming quarks, i.e., questions 2 and 3. However, up-type and down-type quarks in the initial state are distinguished by the parton densities. The relative $u \bar{u}$ and 
$d \bar{d}$ contributions to the $t \bar{t}$ final state are determined by

$$
r(x)=\frac{f_{u}(x) f_{\bar{u}}(s /(x S))}{f_{d}(x) f_{\bar{d}}(s /(x S))} .
$$

Here $f_{p}(x, s)$ denotes the usual parton distribution of parton $p$ with momentum fraction $x$ of the energy $\sqrt{s} / 2$ in the proton. $\sqrt{S}$ is the hadronic CM energy, and we suppressed the factorization scale choice. Around the valence quark maximum $x \approx 0.1$ the ratio becomes $r \approx 2$. For most observables used in our analysis, the ratio integrated over the relevant phase-space region varies roughly in the range

$$
1.5 \lesssim r \lesssim 3
$$

In what follows we refer to $r$ as (roughly) the relative contribution up partonic up- and down-quark contributions to an observable. In what follows we discuss how the isospin of the incoming quarks can be disentangled in a minimal EFT analysis of $t \bar{t}$ production, neglecting quadratic EFT contributions and NLO QCD corrections.

Let us consider pairs of four-quark operators that are only distinguished by the nature of initial quarks: if the latter are right-handed, as in $O_{t u}^{8}$ and $O_{t d}^{8}, t \bar{t}$ observables depend on the combination of Wilson coefficients

$$
r C_{t u}^{8}+C_{t d}^{8} \approx 2 C_{t u}^{8}+C_{t d}^{8}
$$

If the initial quarks are left-handed, their nature is only distinguished by a singlet versus triplet $\mathrm{SU}(2)$ structure, as in $O_{Q q}^{1,8}$ and $O_{Q q}^{3,8}$. In this case the typical combination is

$$
(r+1) C_{Q q}^{1,8}+(r-1) C_{Q q}^{3,8} \approx 3 C_{Q q}^{1,8}+C_{Q q}^{3,8} .
$$

The numerical estimate $r \approx 2$ holds for the bulk of the phase space in top pair production. On the other hand, measurements that select highly boosted tops can probe higher parton momentum fractions $x$ and larger ratios $r$, thus constraining different directions in the EFT space.

To illustrate this effect, figure 5 shows bounds on these two pairs of operators obtained from two-dimensional likelihood fit of top-anti-top observables to LHC data.

The red contours use set ' $\mathrm{M}$ ' of table 5 , that contains rates and normalized $m_{t \bar{t}}$ distributions. These observables are most sensitive to quark-antiquark contributions around the maximum of the parton distributions in $x$, where $r \approx 2$. They leave the directions $(1,-2)$ for $\left(C_{t u}^{8}, C_{t d}^{8}\right)$ and $(1,-3)$ for $\left(C_{Q q}^{1,8}, C_{Q q}^{3,8}\right)$ essentially unbounded, as is expected from the relations in eqs. (4.8) and (4.9).

Boosted top pair production [22] probes larger momentum fractions $x$ and hence larger ratios $r$. The black contours in figure 5 show the likelihood obtained by fitting the last bins of a $p_{T, t}$ distribution in the boosted regime, $p_{T, t}>500 \mathrm{GeV}$. The blind directions of this fit are tilted compared to the previous analysis. They run roughly along $(1,-3)$ for $\left(C_{t u}^{8}, C_{t d}^{8}\right)$ and along $(1,-2)$ for $\left(C_{Q q}^{1,8}, C_{Q q}^{3,8}\right)$, which corresponds to $r \approx 3$. Adding boosted top-antitop observables thus breaks the blind directions in inclusive top-anti-top production, but only mildly. 

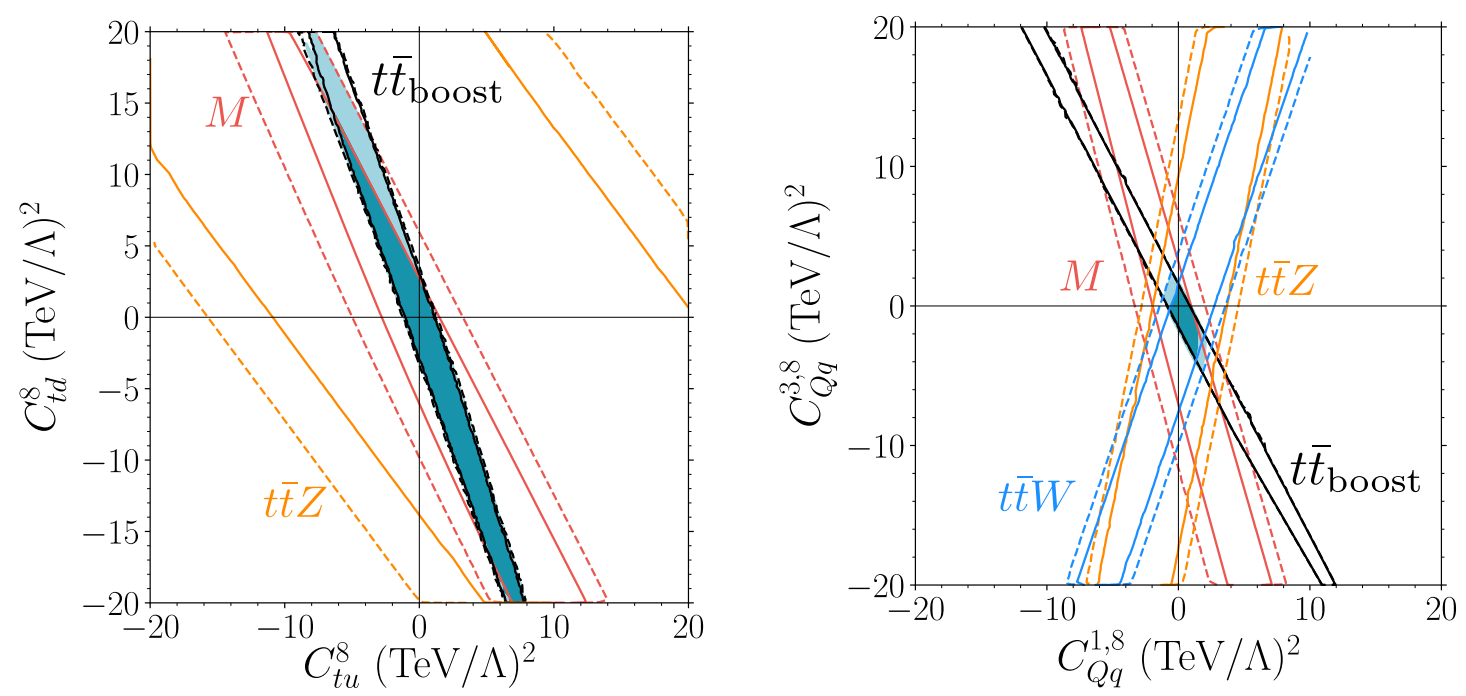

Figure 5. Up-type versus down-type (left) and weak isospin (right) effects of four-quark operators from LO two-parameter fits to order $\Lambda^{-2}$. Solid and dashed lines mark the Gaussian equivalent of $\Delta \chi^{2}=1,4$ from fits to: set $M$ of $t \bar{t}$ observables (red, see table 5 ), highest-energy bins of a $t \bar{t}$ distribution in the boosted regime, $t \bar{t} Z$ rates (orange), $t \bar{t} W$ rates (blue). The shaded areas show the combined fit.

To better resolve the weak gauge structure, we include $t \bar{t} Z$ and $t \bar{t} W$ production in the fit. As mentioned in section 2.3, the radiation of the gauge boson changes the relative contributions of operators with different weak gauge structure. For operators with righthanded light quarks, only $t \bar{t} Z$ production is relevant. At $O\left(\Lambda^{-2}\right)$, the contribution to the $t \bar{t} Z$ rate depends on the Wilson coefficients as

$$
\begin{aligned}
\sigma_{t \bar{t} Z}^{\mathrm{int}}= & \left(r C_{t u}^{8}+C_{t d}^{8}\right) \sigma_{f f}+\left(r\left|g_{u Z}^{R}\right|^{2} C_{t u}^{8}+\left|g_{d Z}^{R}\right|^{2} C_{t d}^{8}\right) \sigma_{i i} \\
& +\left(r g_{u Z}^{R} C_{t u}^{8}+g_{d Z}^{R} C_{t d}^{8}\right) \sigma_{i f} .
\end{aligned}
$$

The three terms correspond to final-state radiation $\left(\sigma_{f f}\right)$, initial-state radiation $\left(\sigma_{i i}\right)$, and interference between initial- and final-state radiation $\left(\sigma_{i f}\right)$ of the $Z$-boson. The quark couplings to the $Z$-boson are defined as $g_{u Z}^{R}=-\frac{2}{3} s_{w}^{2}, g_{d Z}^{R}=\frac{1}{3} s_{w}^{2}, g_{u Z}^{L}=\frac{1}{2}-\frac{2}{3} s_{w}^{2}$, and $g_{d Z}^{L}=-\frac{1}{2}+\frac{1}{3} s_{w}^{2}$. The term $\sigma_{f f}$ includes contributions with $Z$ couplings to left- and righthanded top quarks. By comparing with eq. (4.8), we see that $t \bar{t} Z$ production probes a different direction in the $C_{t u}^{8}-C_{t d}^{8}$ parameter space than inclusive $t \bar{t}$ production.

Operators with left-handed quarks and different weak isospin can be probed in both $t \bar{t} Z$ and $t \bar{t} W$ production. In $t \bar{t} Z$ production, they contribute at $O\left(\Lambda^{-2}\right)$ as $^{4}$

$$
\begin{aligned}
\sigma_{t \bar{t} Z}^{\mathrm{int}}= & \left((r+1) C_{Q q}^{1,8}+(r-1) C_{Q q}^{3,8}\right) \sigma_{f f} \\
& +\left(\left(r\left|g_{u Z}^{L}\right|^{2}+\left|g_{d Z}^{L}\right|^{2}\right) C_{Q q}^{1,8}+\left(r\left|g_{u Z}^{L}\right|^{2}-\left|g_{d Z}^{L}\right|^{2}\right) C_{Q q}^{3,8}\right) \sigma_{i i} \\
& +\left(\left(r g_{u Z}^{L}+g_{d Z}^{L}\right) C_{Q q}^{1,8}+\left(r g_{u Z}^{L}-g_{d Z}^{L}\right) C_{Q q}^{3,8}\right) \sigma_{i f} .
\end{aligned}
$$

\footnotetext{
${ }^{4}$ Note that $\sigma_{f f}, \sigma_{i i}$ and $\sigma_{i f}$ are generic symbols for the contributions to the total $t \bar{t} Z$ cross section, so their meaning is different in eq. (4.10) and in eq. (4.11).
} 
In $t \bar{t} W$ production, the parton luminosity for operators with different weak isospin structure is the same, since all operators with left-handed light quarks contribute to the same partonic processes, dominantly $u \bar{d} \rightarrow t \bar{t} W^{+}$and $d \bar{u} \rightarrow t \bar{t} W^{-}$, respectively. Associated $t \bar{t} W^{+}$production probes the following direction in the $C_{Q q}^{1,8}-C_{Q q}^{3,8}$ plane at $O\left(\Lambda^{-2}\right)$,

$$
\begin{aligned}
\sigma_{t \bar{t} W^{+}}^{\mathrm{int}} & =\left(C_{Q q}^{1,8}+C_{Q q}^{3,8}\right) \sigma_{u u}+\left(C_{Q q}^{1,8}-C_{Q q}^{3,8}\right) \sigma_{d d}+C_{Q q}^{3,8} \sigma_{u d} \\
& \approx C_{Q q}^{1,8}\left(\sigma_{u u}+\sigma_{d d}\right)+C_{Q q}^{3,8} \sigma_{u d} .
\end{aligned}
$$

Here $\sigma_{u u}$ and $\sigma_{d d}$ denote cross section contributions where the $W^{+}$boson is radiated off an incoming anti-down or up quark, which probes the operators $O_{Q q}^{1,8}$ and $O_{Q q}^{3,8}$ through their $(\bar{u} u)(\bar{t} t)$ and $(\bar{d} d)(\bar{t} t)$ contributions, respectively. In $\sigma_{u d}$ the $W^{+}$is radiated off a anti-bottom quark in the final state, probing $O_{Q q}^{3,8}$ through its $(\bar{d} u)(\bar{t} b)$ contribution. The contribution of $O_{Q q}^{3,8}$ largely cancels between $\sigma_{u u}$ and $\sigma_{d d}$, so that the total cross section is sensitive to $O_{Q q}^{3,8}$ mostly through final state radiation. Very similar considerations hold for $t \bar{t} W^{-}$production. In summary, $t \bar{t} W$ production probes a third direction in the $C_{Q q}^{1,8}-C_{Q q}^{3,8}$ plane, in addition to $t \bar{t}$ and $t \bar{t} Z$ production.

In figure 5, we show the impact of cross section measurements at $13 \mathrm{TeV}$ for $t \bar{t} Z$ (orange) and $t \bar{t} W$ (blue). For the $R R$ operators $C_{t u}^{8}$ and $C_{t d}^{8}$ (left panel), $t \bar{t} Z$ production probes indeed a different direction than inclusive $t \bar{t}$ production, leaving a band along $(1,-0.8)$ unconstrained. However, the sensitivity of the $t \bar{t} Z$ cross section to $R R$ operators is much lower than in $t \bar{t}$ production. In the combined fit, shown as a blue area, the remaining blind direction is thus aligned with boosted top pair production. Differential $t \bar{t} Z$ distributions can help to resolve this direction, featuring a better sensitivity to four-quark operators at high energies, similar to $t \bar{t}$ production [80].

The situation is different for $L L$ operators, as we show in the right panel of figure 5 . Associated $t \bar{t} Z$ and $t \bar{t} W$ production probe similar directions in $\left(C_{Q q}^{1,8}, C_{Q q}^{3,8}\right)$, leaving blind directions along roughly $(1,4.7)$ and $(1,2.8)$ respectively. Remarkably, the sensitivity of $t \bar{t} Z$ and $t \bar{t} W$ cross sections to $L L$ operators is comparable to that of differential $t \bar{t}$ distributions. In $t \bar{t} W$ production, both SM and dimension- 6 contributions are induced by quark-antiquark interactions. Compared to the SM rate, effects of $L L$ operators are thus larger than in $t \bar{t}$ production, which is dominated by gluon-gluon interactions. In $t \bar{t} Z$ production the sensitivity to $L L$ operators is much larger than for $R R$ operators. This is due to the different $Z$-couplings to left- and right-handed quarks, $\left|g_{u Z}^{L}\right| /\left|g_{u Z}^{R}\right| \approx 2.4$ and $\left|g_{d Z}^{L}\right| /\left|g_{d Z}^{R}\right| \approx 5.8$, which affect the operator contributions, see eqs. (4.10) and (4.11). This makes $t \bar{t} W$ and $t \bar{t} Z$ production valuable probes of $L L$ four-quark operators, complementary to $t \bar{t}$ production.

\subsection{Top chirality from charge asymmetry}

One way to directly access the chiral structure of four-quark operators is through observables like charge asymmetries, as discussed in section 2.1. At the LHC it has been measured in terms of absolute top and anti-top rapidities,

$$
A_{C}=\frac{\sigma(\Delta|y|>0)-\sigma(\Delta|y|<0)}{\sigma(\Delta|y|>0)+\sigma(\Delta|y|<0)} \quad \text { with } \quad \Delta|y|=\left|y_{t}\right|-\left|y_{\bar{t}}\right| .
$$



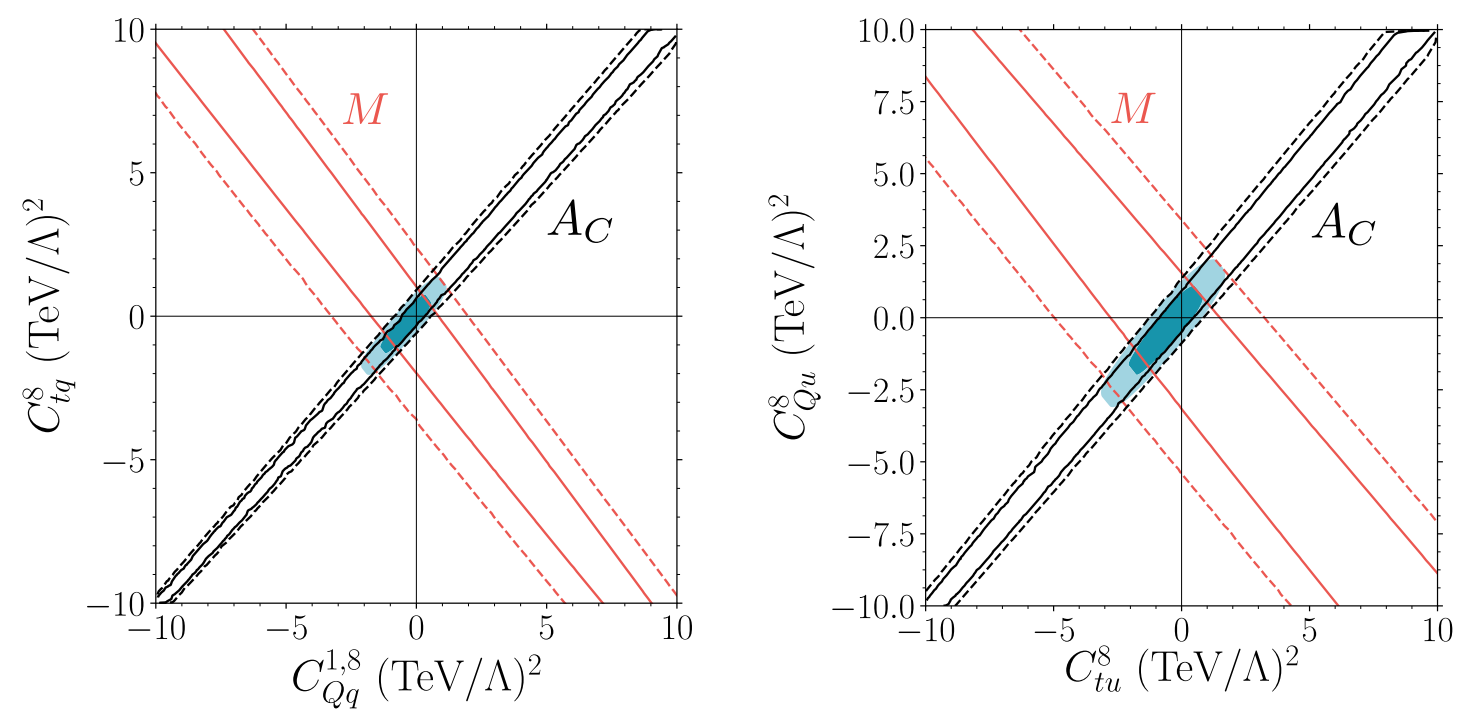

Figure 6. Chirality effects of four-quark operators from LO two-parameter fits to order $\Lambda^{-2}$. Red lines use charge-symmetric observables (set $\mathrm{M}$ of table 5) while black lines use asymmetries $A_{C}$. The shaded areas show the combined fit. Solid and dashed lines mark the Gaussian equivalent of $\Delta \chi^{2}=1,4$.

In QCD such an asymmetry arises only at NLO. In SMEFT, it is induced at LO by fourquark contributions. For illustration, we consider the two operators $O_{Q q}^{1,8}$ and $O_{t q}^{8}$ with a left-handed light-quark current and different chirality of the top current. Since both operators are weak singlets, there is no distinction between up and down quarks. Now the chiral coefficients from eq. (2.10) are given by

$$
4 C_{V V}^{q, 8}=C_{Q q}^{1,8}+C_{t q}^{8}=-4 C_{V A}^{q, 8}, \quad 4 C_{A A}^{q, 8}=C_{Q q}^{1,8}-C_{t q}^{8}=-4 C_{A V}^{q, 8} .
$$

To leading order QCD, the charge asymmetry depends on the corresponding Wilson coefficients as

$$
A_{C}=\frac{\sigma_{A A}\left(C_{Q q}^{1,8}-C_{t q}^{8}\right)}{\sigma_{\mathrm{SM}}+\sigma_{V V}\left(C_{Q q}^{1,8}+C_{t q}^{8}\right)} .
$$

Here $\sigma_{\mathrm{SM}}$ is the SM $t \bar{t}$ rate, $\sigma_{V V}$ and $\sigma_{A A}$ denote the contributions proportional to $4 C_{V V}$ and $4 C_{A A}$ (see eq. (2.12)), and the sum over all $q \bar{q}$ parton contributions is implicit. This expression is easily inferred from eq. (2.12), observing that the charge asymmetry probes the linear terms in $c_{t}$ in the partonic cross section. From the definition of $C_{A A}$ in eq. (2.10), we also see that $A_{C}$ is sensitive to $(L L-R L)+(R R-L R)$, thereby distinguishing between left- and right-handed top quarks.

For the operator pair we have chosen, charge-symmetric observables probe the $(1,1)$ direction in $\left(C_{Q q}^{1,8}, C_{t q}^{8}\right)$, which corresponds to a vector-like top coupling. The charge asymmetry is sensitive to the $(1,-1)$ direction, which corresponds to an axial-vector-like top coupling. The corresponding flat directions can be seen in the left panel of figure 6 , where we show bounds on the Wilson coefficients $\left(C_{Q q}^{1,8}, C_{t q}^{8}\right)$ from a fit to measurements of total 

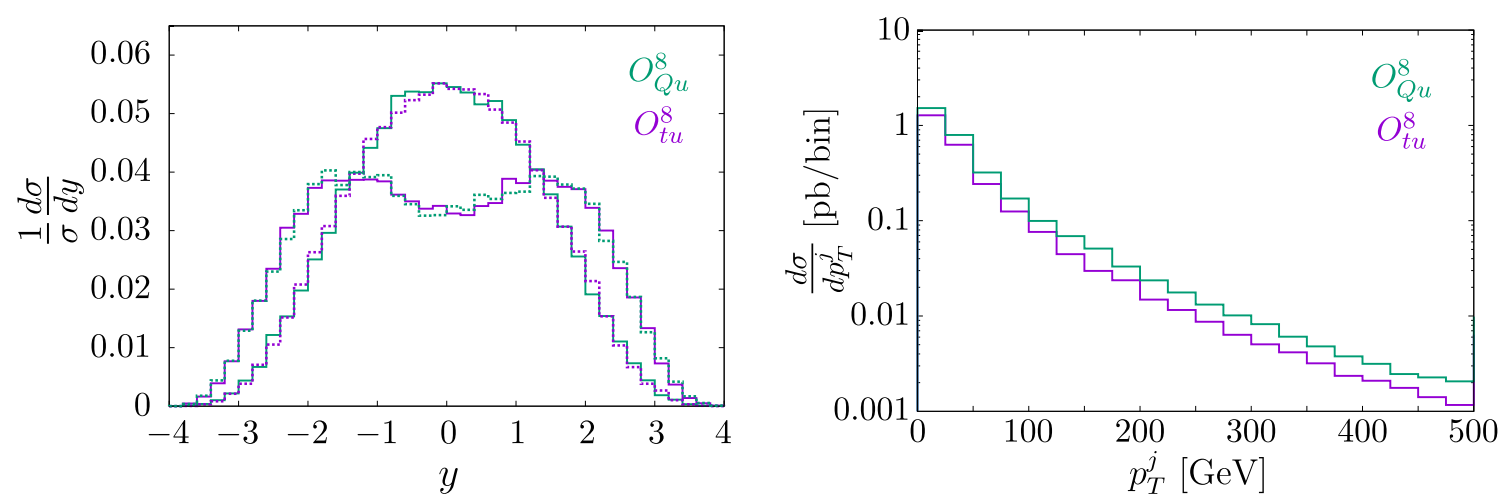

Figure 7. Left: rapidity of top (plain curves) and anti-top (dashed curves) in $p p \rightarrow t \bar{t}$ for the SM-interference of the $L R$ (green) and $R R$ (purple) four-quark operators. Right: jet transverse momentum distribution in $p p \rightarrow t \bar{t} j$ for the SM-interference.

cross sections and $m_{t \bar{t}}$ distributions labelled ' $\mathrm{M}$ ' in table 5 (red lines) and of the charge asymmetries $A_{C}$ (black lines). The shaded blue region shows the combined fit with both datasets, which probes both vector and axial-vector currents with top quarks and breaks the respective blind direction in $\sigma$ or $A_{C}$.

The same behavior applies to operators with right-handed initial quarks, like $O_{t u}^{8}$ and $O_{Q u}^{8}$. As shown in figure 6, right, their effects on $\sigma$ and $A_{C}$ at order $\Lambda^{-2}$ are the same as in eq. (4.14), just replacing $C_{Q q}^{1,8} \rightarrow C_{t u}^{8}, C_{t q}^{8} \rightarrow C_{Q u}^{8}$.

\subsection{Top chirality from jet radiation}

As an alternative to the asymmetry in the previous section we can also use patterns in QCD jet radiation to distinguish four-quark operators with different chirality structures. For instance the operators $O_{t u}^{8}(R R)$ and $O_{Q u}^{8}(L R)$ differ only in the chirality of the top quark. Their leading contribution to top pair production is the same for the inclusive rate and for any charge-symmetric observable, which probe $C_{V V}^{u, 8} \propto C_{t u}^{8}+C_{Q u}^{8}$ and $\left|C_{V+A}^{u, 8}\right|^{2} \propto$ $\left|C_{t u}^{8}\right|^{2}+\left|C_{Q u}^{8}\right|^{2}$, cf. eq. (2.10). However, the two operators are distinguishable in top rapidity distributions, as shown in the left panel of figure 7.

Here $O_{t u}^{8}$ gives more forward or backward tops, compared to $O_{Q u}^{8}$ which leads to more central tops. These different rapidity distributions are directly related to the angular distribution of the top quark in the CM frame of the collision (cf. eq. (2.12)),

$$
\begin{aligned}
\frac{d \sigma(u \bar{u} \rightarrow t \bar{t})}{d \cos \theta_{t}} \propto & \left(1+2 \beta_{t \bar{t}} \cos \theta_{t}+4 \bar{m}^{2}+\beta_{t \bar{t}}^{2} \cos ^{2} \theta_{t}\right) C_{t u}^{8} \\
& +\left(1-2 \beta_{t \bar{t}} \cos \theta_{t}+4 \bar{m}^{2}+\beta_{t \bar{t}}^{2} \cos ^{2} \theta_{t}\right) C_{Q u}^{8}
\end{aligned}
$$

where $\theta_{t}$ is the angle between the incoming up quark and the top. In that sense the contribution of the $R R$ operator is 'forward' whilst the $L R$ operator contributes as 'backward'.

Combined with the color structure this directionality implies that an additional jet can break the degeneracy of the two operators. In the hard process $q \bar{q} \rightarrow t \bar{t}$ the triplet color charge flows from the incoming quark to the top quark and from the anti-quark to 

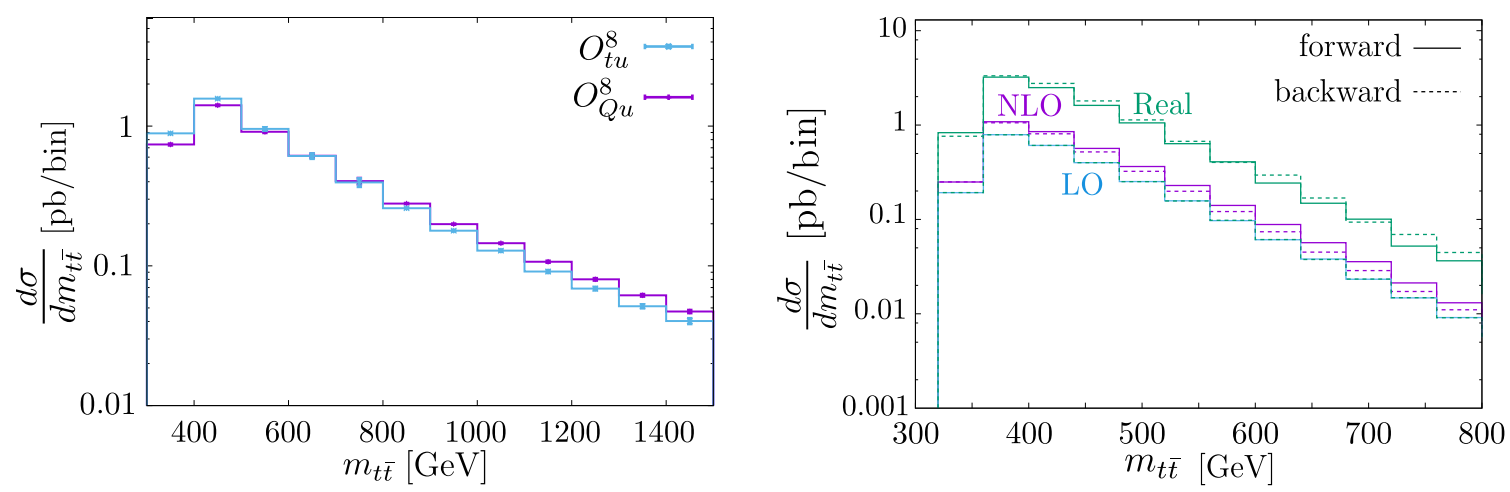

Figure 8. Left: top pair invariant mass distribution in $p p \rightarrow t \bar{t}$ at NLO for the SM-interference of the $R R$ and $L R$ operators. Right: top pair invariant mass distribution in $p \bar{p} \rightarrow t \bar{t}$ at NLO in QCD. By 'forward' we denote events with $y_{t}>0$ and by 'backward' events with $y_{t}<0$.

the anti-top. This leads to a stronger acceleration of color, and consequently more QCD radiation, when the top is produced backwards compared to forwards in the $q \bar{q}$ frame. The same effect can be seen in the context of the top rapidity asymmetry [118]. The additional radiation when the top is backwards pushes the recoiling top-anti-top pair to higher transverse momentum. Indeed, in the right panel of figure 7 we find that $O_{Q u}^{8}$ gives a harder jet $p_{T}$ distribution than $O_{t u}^{8}$. The same effect can be seen in the invariant mass distribution, where $O_{Q u}^{8}$ gives a harder $m_{t \bar{t}}$ distribution.

The jet kinematics of the operator contributions illustrate the impact of NLO corrections in inclusive top-anti-top production. At NLO both the real and virtual corrections break the operator degeneracy in the $t \bar{t}$ distributions. The invariant mass distribution in $t \bar{t}$ production at NLO is shown in the left panel of figure 8. Now the $R R$ operator $O_{t u}^{8}$ gives the harder distribution, implying that the virtual corrections have a large effect in the opposite direction of the real emission. The difference between the $L R$ and $R R$ operators at NLO reaches $20 \%$ in the distributions.

To clarify the interplay between virtual and real corrections, we perform a comparison between forward and backward tops in QCD. For a cleaner comparison, we use $p \bar{p}$ collisions that are dominated by the $q \bar{q}$ partonic initial state. We define forward top quarks as emitted in the direction of the proton and use positive and negative rapidities to define forward and backward tops. In the right panel of figure 8 we show the NLO distributions in $p \bar{p} \rightarrow t \bar{t}$ separately for forward and backward tops. The results confirm that real radiation behaves differently from the total rate at NLO, given by the sum of Born, virtual and real corrections. This means that NLO QCD corrections break the degeneracy of operators that occurs at LO. Our example demonstrates the potential of using NLO QCD corrections more generally to distinguish between operators.

\subsection{Quadratic terms and flat directions}

The dependence of the observables on effective operators changes significantly if we include contributions to order $\Lambda^{-4}$. This is particularly true for four-quark operators that do not interfere with the SM amplitude to leading order because of their color or helicity structure. 
For these operators, quadratic contributions can be the leading effect in an observable. For operators that interfere with the SM, quadratic terms can change the bounds from LHC measurements significantly, for instance in case of strong cancellations between linear and quadratic contributions or in case of limited sensitivity. A dominance of the quadratic term for a specific operator is thus per se not a problem with the convergence of the effective theory, as it can be due to a distinctive physics pattern which suppresses the naively leading contribution. In general, an effective field theory approach is justified if a heavy particle can be decoupled for a given observable.

As an illustration of the role of quadratic terms in our analysis we look again at the operators $O_{Q q}^{1,8}$ and $O_{Q q}^{3,8}$, for which the $t \bar{t}$ cross section and other charge-symmetric observables depend on the Wilson coefficients as

$$
\begin{aligned}
\sigma_{t \bar{t}}=\sigma_{\mathrm{SM}} & +\sigma_{V V}^{d}\left[r\left(C_{Q q}^{1,8}+C_{Q q}^{3,8}\right)+\left(C_{Q q}^{1,8}-C_{Q q}^{3,8}\right)\right] \\
& +\sigma_{V+A}^{d}\left[r\left(C_{Q q}^{1,8}+C_{Q q}^{3,8}\right)^{2}+\left(C_{Q q}^{1,8}-C_{Q q}^{3,8}\right)^{2}\right],
\end{aligned}
$$

where $\sigma_{V V}^{d}$ and $\sigma_{V+A}^{d}$ are the contributions from the partonic $d \bar{d} \rightarrow t \bar{t}$ process. As discussed in section 4.2 , the linear terms to order $\Lambda^{-2}$ have a flat direction which can be resolved using the kinematic variation of the parton densities. From figure 5 we learn that the latter have only limited discriminating power, leaving values $C / \Lambda^{2} \approx \pm 10 / \mathrm{TeV}^{2}$ within the allowed range. In this region, contributions from the squared dimension- 6 amplitudes, i.e., the terms in the second line of eq. (4.17), are numerically dominant.

Due to the presence of quadratic terms of order $\Lambda^{-4}$ any rate prediction $d \sigma$ is positive even for large Wilson coefficients. This implies that in a fit of the two-dimensional parameter space $\left(C_{Q q}^{1,8}, C_{Q q}^{3,8}\right)$, we can set an upper bound in any direction. From the second line of eq. (4.17) we can immediately read off that there still exists a flat direction, where the cross section remains constant for varying Wilson coefficients. In contrast to the linearized case this flat direction forms an ellipse, which we can collapse into any direction to derive a finite limit on the individual coefficients.

This argument also applies to more than two parameters, and has a simple geometric interpretation. For each observable, the points in the $n$-dimensional fit space where this has a given constant value form a $(n-1)$-dimensional hyper-surface. The shape of this hyper-surface is fixed by the EFT parameterization: in the case of (differential) rate measurements, that are positive-definite, it is always a compact manifold, ie. a hyper-ellipsoid. Any such measurement therefore induces a radial constraint on the parameter space, and the viable region identified is necessarily compact. In this sense, including the quadratic terms does not reduce the dimension of the parameter space, but rather changes the topology of the likelihood function. In particular, blind directions in the parameter space are not broken, but "compactified".

In the left panel of figure 9 we show the same fit of $\left(C_{Q q}^{1,8}, C_{Q q}^{3,8}\right)$ as in the right panel of figure 5, but including dimension- 6 squared terms in the predictions. The elliptic shape of the bounds reflects the geometric dependence of the observables on the two Wilson coefficients. This is in contrast with the linear fit from figure 5, where the combined bound had a diamond shape. It is interesting to compare the respective sensitivity of the linear and 

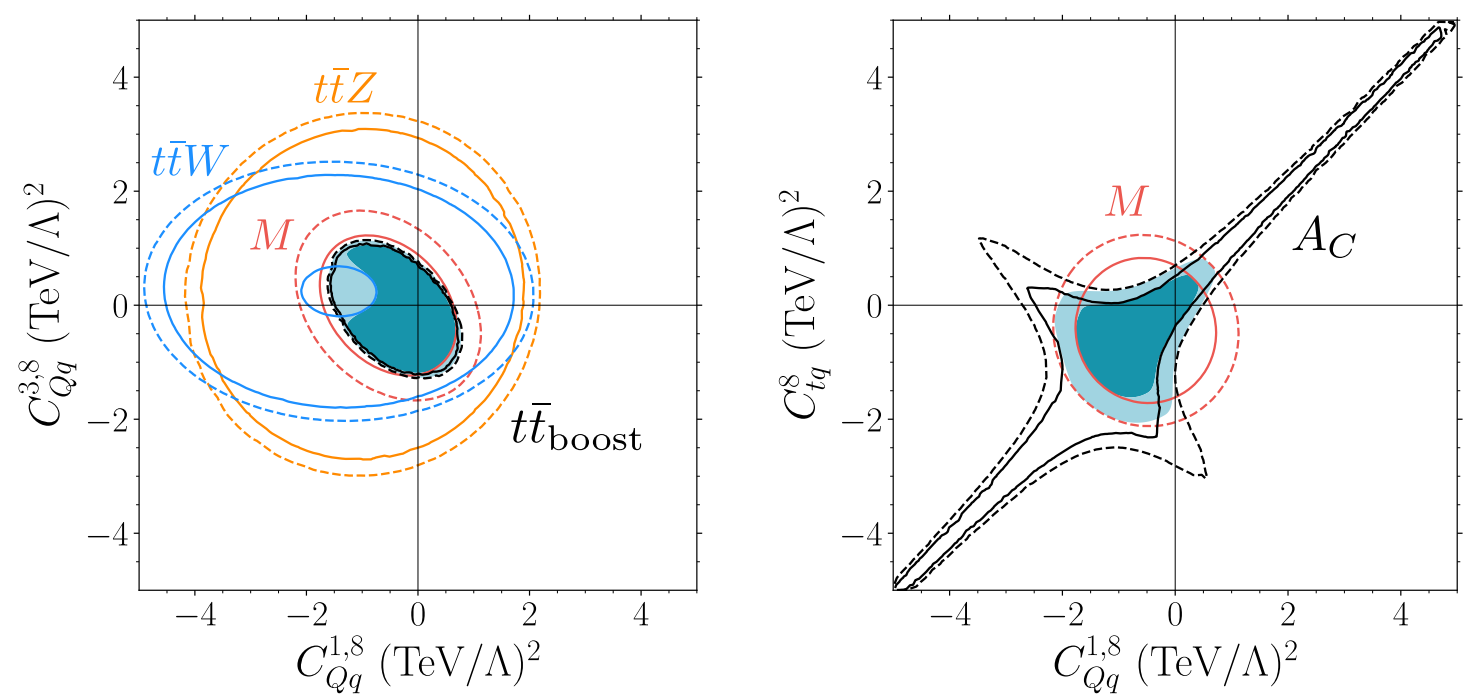

Figure 9. Impact of the squared dimension- 6 contribution on the fit result originally shown for the isospin distinction in figure 5 (right) and for the chirality distinction in figure 6 (left). The lines are based on the same $t \bar{t}$ data set as before, but the predictions now include SMEFT contributions to order $\Lambda^{-4}$ for $t \bar{t}$ (red), $t \bar{t} Z$ (orange) and $t \bar{t} W$ (blue). The black lines show the fit for symmetric observables in the boosted regime (left) and for the asymmetries $A_{C}$ (right) to order $\Lambda^{-4}$. Shaded areas show the combined fit to order $\Lambda^{-4}$. Solid and dashed lines mark the Gaussian equivalent of $\Delta \chi^{2}=1,4$.

quadratic fits. For $t \bar{t}$ production alone, quadratic contributions induce a drastically stronger bound on the individual operators. We can also see that when quadratic terms are included $t \bar{t} Z$ and $t \bar{t} W$ rates (orange and blue ellipses) play a minor role in resolving blind directions compared to the linear case. In fact the combined fit result (blue area) is dominated by the quadratic contributions in boosted $t \bar{t}$ observables (black ellipse). This illustrates nicely the interplay of linear and quadratic contributions in a global fit. The bound on individual Wilson coefficients can be set either by quadratic terms in the dominant observable (for limited sensitivity) or by the interplay of linear terms in several observables that probe different directions of the parameter space (for high sensitivity). Which effect dominates depends on the overall sensitivity of the observables to operator contributions and on the precision of their measurement.

A different geometrical behavior can be observed for instance in the case of the charge asymmetry in $\bar{t} t$ production. Unlike rates, this observable is not positive-definite so that negative quadratic contributions to $A_{C}$ can generally occur. As a consequence, the hypersurfaces of constant $A_{C}$ in the parameter space are in general not compact. For instance, for the chiral operators $O_{Q q}^{1,8}$ and $O_{t q}^{8}$, the cross section and the asymmetry read

$$
\begin{aligned}
& \sigma_{t \bar{t}}=\sigma_{\mathrm{SM}}+\sigma_{V V}\left(C_{Q q}^{1,8}+C_{t q}^{8}\right)+\sigma_{V+A}\left(\left|C_{Q q}^{1,8}\right|^{2}+\left|C_{t q}^{8}\right|^{2}\right)+\sigma_{V-A} C_{Q q}^{1,8} C_{t q}^{8}, \\
& A_{C}=\frac{\sigma_{\mathrm{SM}}^{A}+\sigma_{A A}\left(C_{Q q}^{1,8}-C_{t q}^{8}\right)+\sigma_{V V A A}\left(\left|C_{Q q}^{1,8}\right|^{2}-\left|C_{t q}^{8}\right|^{2}\right)}{\sigma_{t \bar{t}}}
\end{aligned}
$$


A fit of charge-symmetric observables leads to a spherical bound in $\left(C_{Q q}^{1,8}, C_{t q}^{8}\right)$, shown as red curves in the right panel of figure 9. For the charge asymmetry the isocurves are hyperbolas with asymptotes along the directions $(1,1)$ and $(1,-1)$. The fit results reflect this shape in the black curves and leave the direction $(1,1)$ unconstrained. The fact that the direction $(1,-1)$ is bounded is due to the combination of asymmetry measurements with different best-fit points.

\section{Single top analysis}

In addition to the top pair observables described in the previous section our global top analysis also includes single top production. Some Feynman diagrams for the different processes are shown in figure 2. The structure of the single top sector is very similar to classic global SMEFT analyses in that the operators listed in table 1 have distinctive observable effects and can be probed with the sizeable number of different measurements listed in table 6. Flat directions are not an issue in this sector, but it is interesting to test if there exist correlations in the bounds on the individual operators.

We evaluate all two-operator correlations based on two-dimensional profile likelihoods and find three distinct patterns shown in the upper row of figure 10. First, a box shape like for $C_{t G}$ and $C_{Q q}^{3,8}$ appears if two Wilson coefficients are bounded by two separate sets of observables. Next, an elliptic disk like the one between $C_{b W}$ and $C_{\phi t b}$ appears if two operators contribute quadratically to the same observable. Finally, a shifted circle like in the $C_{Q q}^{3,1}-C_{Q q}^{3,8}$ plane appears if two operators contribute to the same observables, but one of them linearly $\left(C_{Q q}^{3,1}\right)$ and the other one only quadratically $\left(C_{Q q}^{3,8}\right)$. For this pattern the SM value cannot be at the center of the circle.

One of the few noteworthy correlations in the single top fit is the inverted heart shape in the $C_{t G}-C_{\phi Q}^{3}$ plane shown in the lower left panel of figure 10. It can be understood as the interplay of the three operators $C_{t G}, C_{\phi Q}^{3}$, and $C_{Q q}^{3,1}$ with at least two measurements. The only single top measurement sensitive to $O_{t G}$ is $t W$ production. Using its rate to constrain $C_{t G}$ and $C_{\phi Q}^{3}$ we find an elliptic correlation centered at negative values of $C_{\phi Q}^{3}$. When we add the strong constraints on $C_{\phi Q}^{3}$ from $t$-channel production the bottom part of the ellipsis gets removed. Finally, once we add $C_{Q q}^{3,1}$ to the fit we find that $O_{\phi Q}^{3}$ and $O_{Q q}^{3,1}$ are slightly correlated and hence more negative values of $O_{\phi Q}^{3}$ become consistent with data. In the lower panels of figure 10 we project the 3 -dimensional profile likelihood from a 3-parameter fit along each of the three directions. In the left panel we see a very faint barrier for $C_{\phi Q}^{3} / \Lambda^{2} \approx 1.5 \mathrm{TeV}^{-2}$. It corresponds to the two disconnected regions, one for $C_{Q q}^{3,1} / \Lambda^{2} \approx 0$ and one for $C_{Q q}^{3,1} / \Lambda^{2} \approx 0.4 \mathrm{TeV}^{-2}$, which we see clearly in the central and right panels. In the global single top fit, once all observables are included, only the region for $C_{\phi Q}^{3} / \Lambda^{2} \approx 0$ remains, while the other region becomes disfavored.

Given the smooth behavior of the multi-dimensional likelihood we can perform a global fit of the single top sector, including the $W$ helicity fractions in top decay and associated $t V$ production. The one-dimensional profile likelihoods are shown in figure 11 . The only non-standard aspect in these results is that we cannot define meaningful $68 \%$ CL limits for 

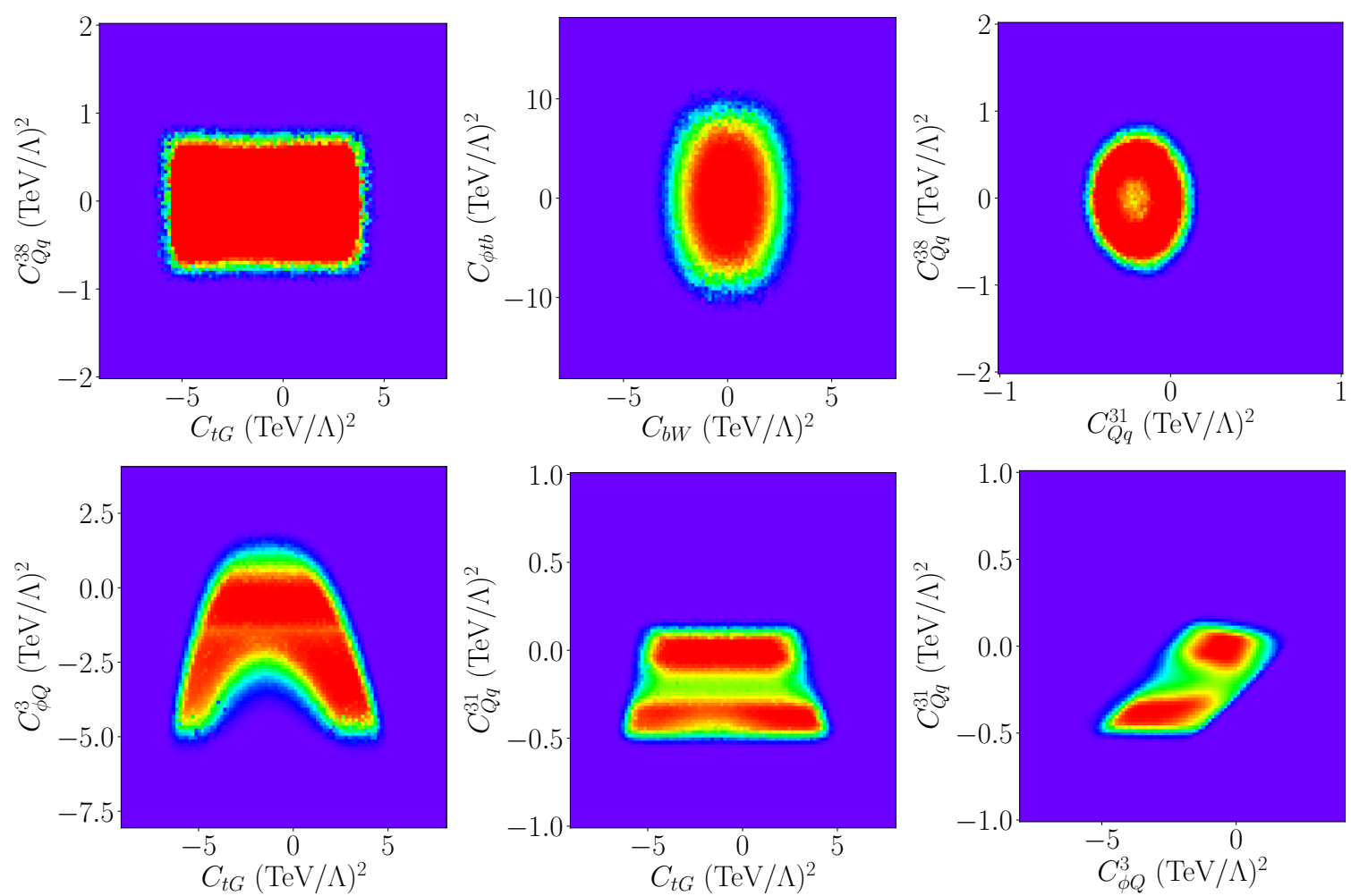

Figure 10. Upper: examples for correlated 2-dimensional profile likelihoods of operators in a global fit to the single top data. Lower: correlated profile likelihoods for a three-parameter fit of the same data.

some of the operators. This happens when a flat core of the profile likelihood covers more than $68 \%$ of the integral and there exists no unique definition of a range. We observe this for all operators except for $O_{\phi t b}, O_{t W}$, and $O_{b W}$, implying that for all other operators the theory uncertainty is large compared to the experimental statistics and systematics.

One aspect which sticks out in the global fit is the low sensitivity to $O_{\phi Q}^{3}$, compared to $O_{Q q}^{3,1}$ and $O_{t W}$. All three operators interfere with the SM amplitude in $t$-channel single top production, but for $O_{\phi Q}^{3}$ the effect is numerically smaller by about a factor three. As discussed in section 2.2, $O_{\phi Q}^{3}$ only rescales the SM contribution, while $O_{Q q}^{3,1}$ changes the kinematics in $t$-channel production, see table 2. The operator $O_{t W}$ is best constrained by the $W$ helicity fractions in top decay, see eq. (2.18), which are very sensitive to this operator.

The bounds on $C_{Q q}^{3,8}, C_{\phi t b}$ and $C_{b W}$ are symmetric around zero, since the corresponding operators contribute to single top observables only at order $\Lambda^{-4}$, cf. table 1 . The coefficients $C_{\phi Q}^{-}, C_{t Z}$ and $C_{\phi t}$ are bound by $t Z$ production. Due to the limited experimental precision, the bounds on these operators are very loose. Also here the SM-interference plays a role, leading to asymmetric bounds for $C_{\phi Q}^{-}$and $C_{\phi t}$. The sensitivity to $O_{\phi t}$ is especially poor because its contribution to $t Z$ production is suppressed, see section 2.2. This will change once we include the better-measured $t \bar{t} Z$ channel in the global fit. 


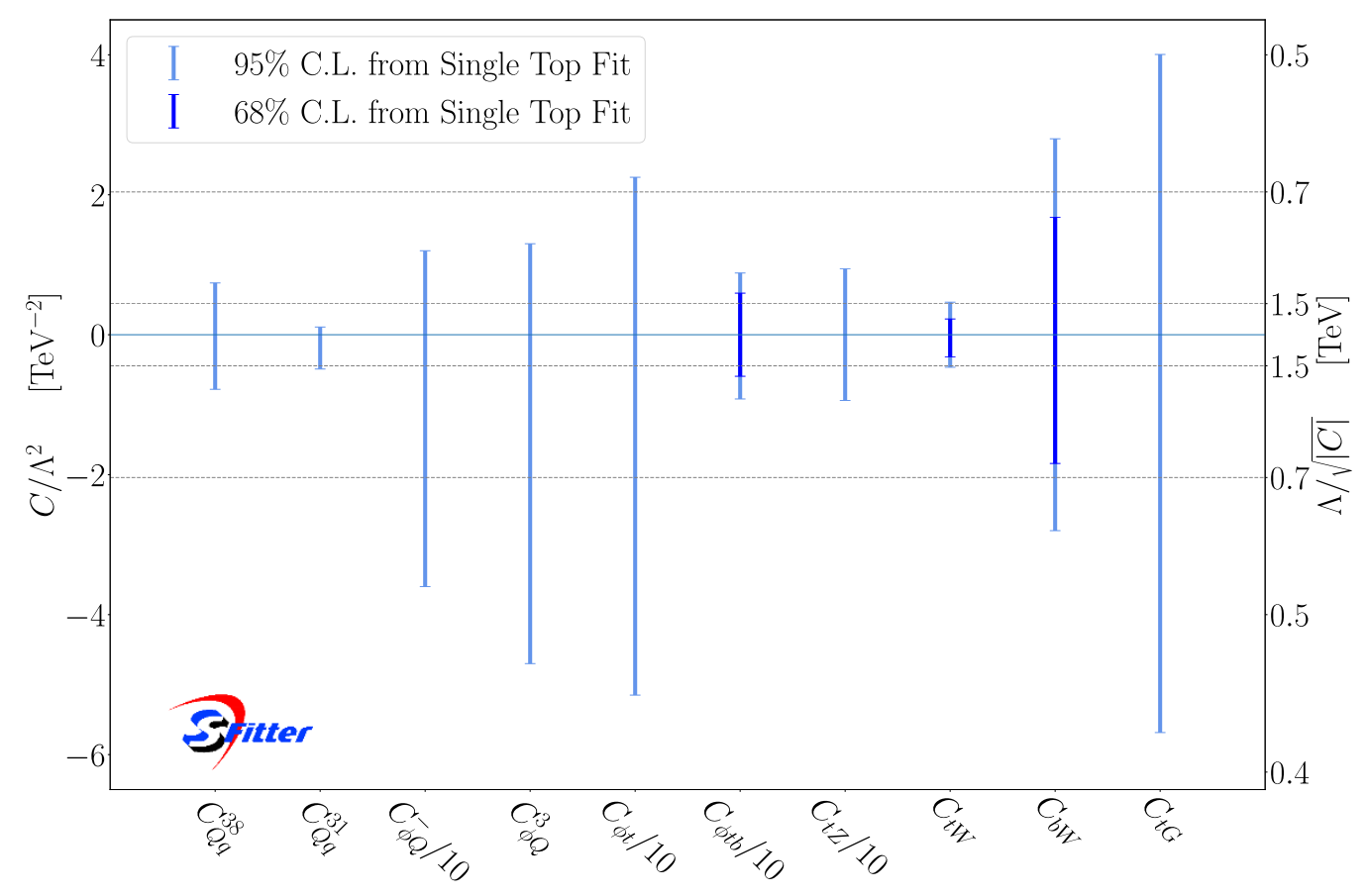

Figure 11. $95 \%$ and $68 \%$ CL bounds for the global fit to the single top data set from table 6 . Whenever the $68 \%$ CL is not shown it falls into the flat profile likelihood regime reflecting dominant theoretical uncertainties.

\section{Global top analysis}

In the final step we add all top pair measurements from table 5 to our single top fit based on the measurements in table 6 and presented in section 5. On the parameter side we add the large number of four-quark operators, which roughly doubles the number of model parameters. For the measurements we not only include top pair production, but also associated $t \bar{t} W$ and $t \bar{t} Z$ rate measurements. They constrain some of the electroweak top operators in single top production and four-quark operators in top pair production, thus linking both sectors in the global fit.

First, we briefly comment on 2-dimensional correlations in the complete fit. The boxshaped correlations for separate operators and separate measurements, filled ellipses for more than one operator affecting a measurement, and shifted circles from linear contributions to compact flat directions which we observed in the single top fit (figure 10) also appear in the global fit.

Non-trivial correlations as between $C_{t G}, C_{\phi Q}^{3}$, and $C_{Q q}^{3,1}$ vanish once we include the full data set, see figure 12 . The reason is that $C_{t G}$ and $C_{Q q}^{3,1}$ are strongly constrained individually by top pair production. For the weak-triplet operators $O_{Q q}^{3,1}$ and $O_{Q q}^{3,8}$ the bounds are the same in the single top fit and the global fit, see also figure 14. Single top production is indeed more sensitive to these four-quark operators than top pair production. 


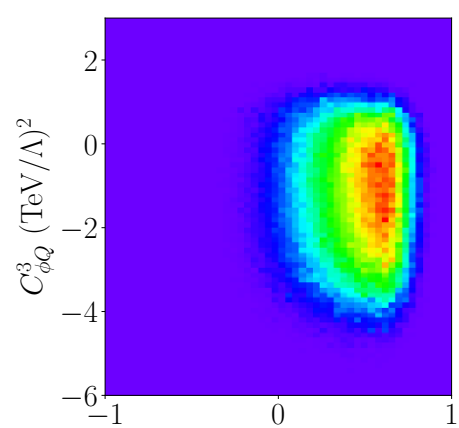

$C_{t G}(\mathrm{TeV} / \Lambda)^{2}$

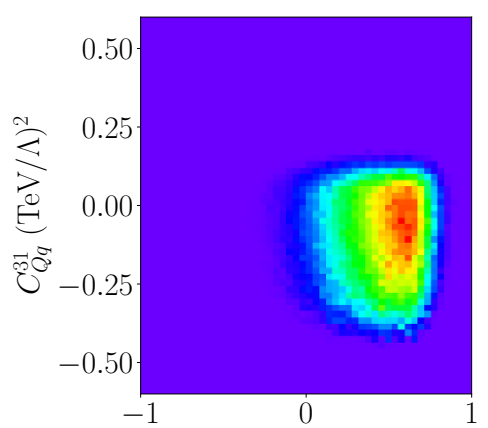

$C_{t G}(\mathrm{TeV} / \Lambda)^{2}$

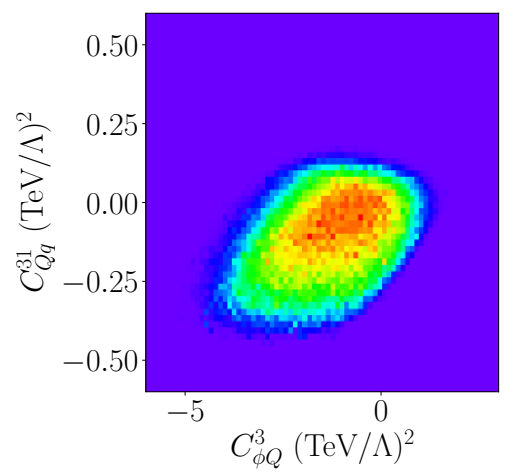

Figure 12. Examples of correlated 2-dimensional profile likelihoods from the global fit, showing the same operators as in the lower panels of figure 10.

Run II, ATLAS+CMS, $68 \%$ and $95 \%$ C.L.

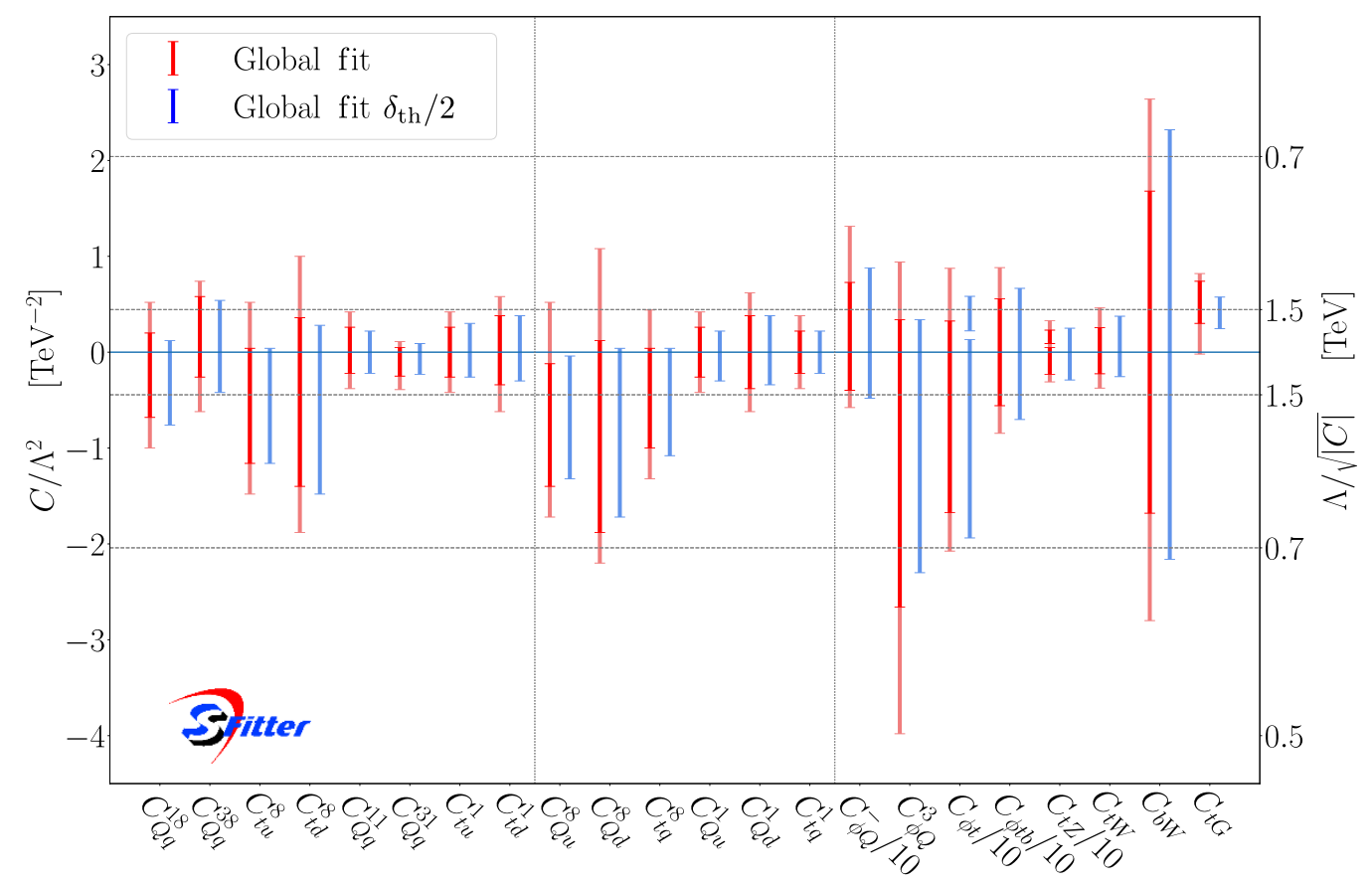

Figure 13. $95 \%$ and $68 \%$ CL bounds on top operators from a global fit to the full data set from tables 5 and 6 . We show the results including all uncertainties (red) and with theoretical uncertainties reduced by a factor of two, $\delta_{\mathrm{th}} / 2$ (blue).

In figure 13 we show the profile likelihoods for each of the top-related effective operators. On the $x$-axis we start with the diagonal $L L$ and $R R$ four-quark operators listed in eq. (2.3), continue with the $L R$ and $R L$ four-quark operators from eq. (2.4), and finally include the bosonic operators from eq. (2.5). For each operator the red bars indicate the final result at $68 \%$ and $95 \% \mathrm{CL}$. These confidence levels are compact intervals defined by the area under the profile likelihood curve, where in addition we require the likelihood values on each side to be equal. For a Gaussian distribution we expect the $95 \%$ error bar to be symmetric around the best-fit value and twice as wide as the symmetric $68 \%$ error 
bar. For some of the Wilson coefficients, non-Gaussian effects occur, which are mainly due to theoretical uncertainties treated as flat likelihoods.

In general, the four-quark operators are extremely well constrained with limits in the range of $\Lambda / \sqrt{C} \approx 1-2 \mathrm{TeV}$. For all weak-singlet four-quark operators, the sensitivity is dominated by high-energy bins in $t \bar{t}$ distributions. For $L L$ operators, we made this observation earlier in the left panel of figure 9. As discussed in section 4.5, for most operators the well-defined limits on each of the four-quark operators rest entirely on the quadratic contributions to the observables. For color-singlet operators, which contribute to top pair production only at order $\Lambda^{-4}$, the bounds are fully determined by quadratic contributions and symmetric around zero. The only asymmetric limit on a color-singlet operator appears for $O_{Q q}^{3,1}$ through a linear contribution to single top production.

Color-octet operators have asymmetric error bars due to their interference with QCD in top pair production. This interference is also the reason for the correlation patterns of shifted circles in figure 9 . The bounds on color-octet operators thus rely on the interplay between contributions of order $\Lambda^{-2}$ and $\Lambda^{-4}$, where the inclusion of both terms is particularly important. In figure 13, the error bars for color-singlet operators appear much smaller than for color-octet operators. This is due to the fact that top-anti-top observables always probe the combination $\left(C^{8}\right)^{2}+\frac{9}{2}\left(C^{1}\right)^{2}$ at order $\Lambda^{-4}$, see eq. (2.12). Top-anti-top observables therefore constrain the quantities $C^{8}$ and $\left(C^{8}\right)^{2}+\frac{9}{2}\left(C^{1}\right)^{2}$ at $\mathrm{LO}$, disentangling color-singlet from color-octet structures in kinematic distributions. The color combination is also changed at NLO in QCD, which in principle offers the possibility to determine the color structure of operators from jet radiation.

Looking at the quark chirality, we observe that the bounds on operators differing only in the top chirality are similar in strength. Charge-symmetric $t \bar{t}$ observables do not distinguish between these operators at high energies, see eqs. (2.10) and (2.12). The charge asymmetry is sensitive to the top chirality, see eq. (4.18), but still leads to equal bounds on the magnitude of $L L$ and $R L$ operators due to its small SM contribution.

Regarding different light quark flavors, operators with up quarks are better constrained than operators with down quarks. This reflects the parton content of the proton, which leads to an enhanced sensitivity of $t \bar{t}$ observables to up-quark operators over down-quark operators, see eq. (4.8).

Let us now turn our attention to the bosonic operators. The strongest bounds are obtained for the dipole operators $O_{t G}$ and $O_{t W}$. For $O_{t W}$ the bound does not change compared to the single top fit (see also figure 14), because it is dominated by the precise measurements of $W$ helicities in top decays. From our global fit, we obtain at 95\% CL

$$
-\frac{1}{(1.6 \mathrm{TeV})^{2}} \lesssim \frac{C_{t W}}{\Lambda^{2}} \lesssim \frac{1}{(1.5 \mathrm{TeV})^{2}} .
$$

For $O_{t G}$ the best global limit at $95 \%$ CL is obtained from top-anti-top production,

$$
-\frac{1}{(5.8 \mathrm{TeV})^{2}} \lesssim \frac{C_{t G}}{\Lambda^{2}} \lesssim \frac{1}{(1.1 \mathrm{TeV})^{2}}
$$

This bound is much stronger than the bound from associated $t W$ production in figure 11. We also note that while the upper limit is consistent with the estimate of eq. (4.4) (which 
however quotes a $68 \% \mathrm{CL}$ ), the lower bound results much stronger, due to the inclusion of constraints from nomalized distributions. This behavior is evident from figure 4, left. Most of the remaining bosonic operators are better constrained in the global fit than in the single top fit (see figure 14). This shows the impact of the $t \bar{t} Z$ cross section measurements in the global fit. For the operator $O_{\phi t b}$, which does not contribute to $t \bar{t} Z$ or $t \bar{t} W$ production, the sensitivity remains very low.

In our fit, theory uncertainties affect the relation between the (rate) measurements and the Wilson coefficients. Since we treat these uncertainties as flat errors in our statistical analysis, they lead to plateaus in the center of the likelihood distributions and to some of the non-Gaussian effects. To study the relative impact of theoretical and experimental uncertainties on the fit results, we have performed a global fit with theory uncertainties divided by a factor of two. The 95\% CL results are shown as blue bars in figure 13. We find that theory uncertainties have a significant impact on the bounds for all the operators, and they are dominant in a few observables.

Reducing the theory uncertainties in the fit is in principle possible, for instance by assessing the uncertainties for the SM and EFT contributions separately or by comparing observables to data at the particle level, thus reducing the uncertainties from unfolding to the parton level. These improvements, however, are computationally costly and depend on the considered observable. We leave them for future work.

\section{Conclusions}

We have presented a comprehensive analysis of the LHC Run II data in the top sector. We use NLO simulations in MADGRAPH5_AMC@NLO and the SFiTTER framework to constrain the Wilson coefficients of 22 dimension- 6 operators. The bulk of the measurements involve final states with a top pair, including kinematic distributions, the charge asymmetry, and associated top pair production with a weak boson. In addition, we include different single top channels and $W$ helicity measurements in top decays. The measurements we use are based on up to $139 \mathrm{fb}^{-1}$ of integrated luminosity.

The main challenge of this global analysis is the large number of four-quark operators in top pair production, whose contribution to the QCD process are largely degenerate. We have discussed several ways of breaking this degeneracy, including parton luminosity effects, the charge asymmetry, jet radiation patterns, and associated production with weak bosons. We have also discussed the impact of dimension-6 squared terms on the fit results, and their role in constraining the viable parameter space.

Altogether, we derive limits in the range of $\Lambda / \sqrt{C}=0.35-2 \mathrm{TeV}$ for the different Wilson coefficients from a profile likelihood. The strongest limit is on the anomalous top coupling to the gluon, driven by the QCD production rate. Similarly strong limits apply to several four-quark operators, stemming mostly from normalized kinematic distributions. The top dipole interaction with the $W$ boson is also strongly constrained by the precisely known $W$ helicity fractions in top decays. Other operators with weak bosons are much less constrained, because they only occur in electroweak top processes with a limited sensitivity in total rates. Differential distributions in electroweak top production, as well as precision observables in electroweak and flavor physics can help to increase the sensitivity. 


\section{Acknowledgments}

We thank Dirk Zerwas for many helpful discussions and experimentalist's advice. The research of SB, TP and SW is supported by the Deutsche Forschungsgemeinschaft (DFG, German Research Foundation) under grant no. 396021762 - TRR 257. SW acknowledges funding by the Carl Zeiss foundation through an endowed junior professorship (JuniorStiftungsprofessur). The authors acknowledge support by the state of Baden-Württemberg through bwHPC and the German Research Foundation (DFG) through grant no INST 39/963-1 FUGG (bwForCluster NEMO). EV is supported by a Marie Skłodowska-Curie Individual Fellowship of the European Commission's Horizon 2020 Programme under contract number 704187. CZ is supported by IHEP under Contract No. Y7515540U1. FM was partly supported by F.R.S.-FNRS under the "Excellence of Science - EOS" - be.h project n. 30820817

\section{A Operator relations}

In this appendix we list the relations between the relevant operators in our analysis and the operators in the Warsaw basis, following the notation of refs. [23, 44]. Using the SU(2) and $\mathrm{SU}(3)$ identities

$$
\tau_{i j}^{I} \tau_{k l}^{I}=-\delta_{i j} \delta_{k l}+2 \delta_{i l} \delta_{j k}, \quad T_{a b}^{A} T_{c d}^{A}=-\frac{1}{6} \delta_{a b} \delta_{c d}+\frac{1}{2} \delta_{a d} \delta_{b c}
$$

and the Fierz identities for anti-commutating fermion fields,

$$
\left(\bar{q} \gamma^{\mu} q\right)\left(\bar{Q} \gamma_{\mu} Q\right)=\left(\bar{q} \gamma^{\mu} Q\right)\left(\bar{Q} \gamma_{\mu} q\right), \quad\left(\bar{u} \gamma^{\mu} u\right)\left(\bar{t} \gamma_{\mu} t\right)=\left(\bar{u} \gamma^{\mu} t\right)\left(\bar{t} \gamma_{\mu} u\right),
$$

we derive the following relations:

- four-quark operators with $L L$ and $R R$ chiral structure $(i=1,2)$,

$$
\begin{aligned}
O_{Q q}^{1,8} & \equiv\left(\bar{Q} \gamma_{\mu} T^{A} Q\right)\left(\bar{q}_{i} \gamma^{\mu} T^{A} q_{i}\right) & & =-\frac{1}{6} \mathcal{O}_{q q}^{1(33 i i)}+\frac{1}{4} \mathcal{O}_{q q}^{1(3 i i 3)}+\frac{1}{4} \mathcal{O}_{q q}^{3(3 i i 3)} \\
O_{Q q}^{3,8} & \equiv\left(\bar{Q} \gamma_{\mu} T^{A} \tau^{I} Q\right)\left(\bar{q}_{i} \gamma^{\mu} T^{A} \tau^{I} q_{i}\right) & & =-\frac{1}{6} \mathcal{O}_{q q}^{3(33 i i)}+\frac{3}{4} \mathcal{O}_{q q}^{1(3 i i 3)}-\frac{1}{4} \mathcal{O}_{q q}^{3(3 i i 3)} \\
O_{Q q}^{1,1} & \equiv\left(\bar{Q} \gamma_{\mu} Q\right)\left(\bar{q}_{i} \gamma^{\mu} q_{i}\right) & & \mathcal{O}_{q q}^{1(33 i i)} \\
O_{Q q}^{3,1} & \equiv\left(\bar{Q} \gamma_{\mu} \tau^{I} Q\right)\left(\bar{q}_{i} \gamma^{\mu} \tau^{I} q_{i}\right) & & =\mathcal{O}_{q q}^{3(33 i i)} \\
O_{t u}^{8} & \equiv\left(\bar{t} \gamma_{\mu} T^{A} t\right)\left(\bar{u}_{i} \gamma^{\mu} T^{A} u_{i}\right) & & =-\frac{1}{6} \mathcal{O}_{u u}^{(33 i i)}+\frac{1}{2} \mathcal{O}_{u u}^{(3 i i 3)} \\
O_{t u}^{1} & \equiv\left(\bar{t} \gamma_{\mu} t\right)\left(\bar{u}_{i} \gamma^{\mu} u_{i}\right) & & \mathcal{O}_{u u}^{(33 i i)} \\
O_{t d}^{8} & \equiv\left(\bar{t} \gamma^{\mu} T^{A} t\right)\left(\bar{d}_{i} \gamma_{\mu} T^{A} d_{i}\right) & & =\mathcal{O}_{u d}^{8(33 i i)} \\
O_{t d}^{1} & \equiv\left(\bar{t} \gamma^{\mu} t\right)\left(\bar{d}_{i} \gamma_{\mu} d_{i}\right) & & \mathcal{O}_{u d}^{1(33 i i)},
\end{aligned}
$$

- four-quark operators with $L R$ and $R L$ chiral structure

$$
\begin{aligned}
O_{Q u}^{8} \equiv\left(\bar{Q} \gamma^{\mu} T^{A} Q\right)\left(\bar{u}_{i} \gamma_{\mu} T^{A} u_{i}\right) & =\mathcal{O}_{q u}^{8(33 i i)} & O_{Q u}^{1} \equiv\left(\bar{Q} \gamma^{\mu} Q\right)\left(\bar{u}_{i} \gamma_{\mu} u_{i}\right) & =\mathcal{O}_{q u}^{1(33 i i)} \\
O_{Q d}^{8} \equiv\left(\bar{Q} \gamma^{\mu} T^{A} Q\right)\left(\bar{d}_{i} \gamma_{\mu} T^{A} d_{i}\right) & =\mathcal{O}_{q d}^{8(33 i i)} & O_{Q d}^{1} \equiv\left(\bar{Q} \gamma^{\mu} Q\right)\left(\bar{d}_{i} \gamma_{\mu} d_{i}\right) & =\mathcal{O}_{q d}^{1(33 i i)} \\
O_{t q}^{8} \equiv\left(\bar{q}_{i} \gamma^{\mu} T^{A} q_{i}\right)\left(\bar{t} \gamma_{\mu} T^{A} t\right) & =\mathcal{O}_{q u}^{8(i i 33)} & O_{t q}^{1} \equiv\left(\bar{q}_{i} \gamma^{\mu} q_{i}\right)\left(\bar{t} \gamma_{\mu} t\right) & =\mathcal{O}_{q u}^{1(i i 33)}
\end{aligned}
$$


- operators with two heavy quarks and bosonic fields

$$
\begin{aligned}
& O_{\phi Q}^{1} \equiv\left(\phi^{\dagger} i \overleftrightarrow{D_{\mu}} \phi\right)\left(\bar{Q} \gamma^{\mu} Q\right) \quad=\mathcal{O}_{\phi q}^{1(33)} \quad{ }^{\ddagger} O_{t B} \equiv\left(\bar{Q} \sigma^{\mu \nu} t\right) \widetilde{\phi} B_{\mu \nu} \quad={ }^{\ddagger} \mathcal{O}_{u B}^{(33)} \\
& O_{\phi Q}^{3} \equiv\left(\phi^{\dagger} i \overleftrightarrow{D_{\mu}^{I}} \phi\right)\left(\bar{Q} \gamma^{\mu} \tau^{I} Q\right)=\mathcal{O}_{\phi q}^{3(33)} \quad{ }^{\ddagger} O_{t W} \equiv\left(\bar{Q} \sigma^{\mu \nu} t\right) \tau^{I} \widetilde{\phi} W_{\mu \nu}^{I} \quad={ }^{\ddagger} \mathcal{O}_{u W}^{(33)} \\
& O_{\phi t} \equiv\left(\phi^{\dagger} i \overleftrightarrow{D_{\mu}} \phi\right)\left(\bar{t} \gamma^{\mu} t\right) \quad=\mathcal{O}_{\phi u}^{(33)} \quad \ddagger O_{b W} \equiv\left(\bar{Q} \sigma^{\mu \nu} b\right) \tau^{I} \phi W_{\mu \nu}^{I} \quad={ }^{\ddagger} \mathcal{O}_{d W}^{(33)} \\
& { }^{\ddagger} O_{\phi t b} \equiv\left(\widetilde{\phi}^{\dagger} i D_{\mu} \phi\right)\left(\bar{t} \gamma^{\mu} b\right) \quad={ }^{\ddagger} \mathcal{O}_{\phi u d}^{(33)} \quad{ }^{\ddagger} O_{t G} \equiv\left(\bar{Q} \sigma^{\mu \nu} T^{A} t\right) \widetilde{\phi} G_{\mu \nu}^{A} \quad={ }^{\ddagger} \mathcal{O}_{u G}^{(33)} \text {, }
\end{aligned}
$$

with the Higgs field $\phi=\left(0, \frac{1}{\sqrt{2}}(v+h)\right)^{\top}$ in unitary gauge, $\widetilde{\phi}=i \sigma_{2} \phi^{*}$ and the covariant derivative

$$
D_{\mu}=\partial_{\mu}-i \frac{e}{2 s_{W}} A_{\mu}^{I} \tau^{I}-i \frac{e}{c_{W}} B_{\mu} Y, \quad D_{\mu}^{I}=\tau^{I} D_{\mu}, \quad \tau^{I}=\sigma_{I}
$$

The relations between the corresponding Wilson coefficients $C_{i}$ and $\mathcal{C}_{i}$ can be obtained by requiring that both bases lead to the same terms in the effective Lagrangian [40],

$$
\mathcal{L}_{\text {eff }}=\sum_{a}\left(\frac{C_{a}}{\Lambda^{2}} \ddagger O_{a}+\text { h.c. }\right)+\sum_{b} \frac{C_{b}}{\Lambda^{2}} O_{b}=\sum_{c}\left(\frac{\mathcal{C}_{c}}{\Lambda^{2}} \ddagger \mathcal{O}_{c}+\text { h.c. }\right)+\sum_{d} \frac{\mathcal{C}_{d}}{\Lambda^{2}} \mathcal{O}_{d} .
$$

After electroweak symmetry breaking, the effective interactions of the physical weak gauge bosons are described by linear combinations of the operators in the unbroken phase. In unitary gauge, the relations read

$$
\begin{aligned}
& \left(\begin{array}{c}
O_{\phi Q}^{1} \\
O_{\phi Q}^{3}
\end{array}\right)=\left(\begin{array}{cccc}
1 & 1 & 0 & 0 \\
-1 & 1 & 1 & 1
\end{array}\right)\left(\begin{array}{c}
-\frac{e}{2 s_{W} c_{W}}\left(\bar{t} \gamma^{\mu} t_{L}\right) Z_{\mu}(v+h)^{2} \\
-\frac{e}{2 s_{W} c_{W}}\left(\bar{b} \gamma^{\mu} b_{L}\right) Z_{\mu}(v+h)^{2} \\
\frac{e}{\sqrt{2} s_{W}}\left(\bar{t} \gamma^{\mu} b_{L}\right) W_{\mu}^{+}(v+h)^{2} \\
\frac{e}{\sqrt{2} s_{W}}\left(\bar{b} \gamma^{\mu} t_{L}\right) W_{\mu}^{-}(v+h)^{2}
\end{array}\right) \\
& \left(\begin{array}{c}
\ddagger O_{t B} \\
\ddagger O_{t W}
\end{array}\right)=\left(\begin{array}{ccc}
c_{W} & -s_{W} & 0 \\
s_{W} & c_{W} & 1
\end{array}\right)\left(\begin{array}{c}
\frac{1}{\sqrt{2}}\left(\bar{t} \sigma^{\mu \nu} t_{R}\right) A_{\mu \nu}(v+h) \\
\frac{1}{\sqrt{2}}\left(\bar{t} \sigma^{\mu \nu} t_{R}\right) Z_{\mu \nu}(v+h) \\
\left(\bar{b} \sigma^{\mu \nu} t_{R}\right) W_{\mu \nu}^{-}(v+h)
\end{array}\right) \text {, } \\
& { }^{\ddagger} O_{b W}=\left[-\frac{1}{\sqrt{2}} \bar{b} \sigma^{\mu \nu} b_{R}\left(c_{w} Z_{\mu \nu}+s_{w} A_{\mu \nu}\right)+\bar{t} \sigma^{\mu \nu} b_{R} W_{\mu \nu}^{+}\right](v+h) \text {. }
\end{aligned}
$$

\section{B Numerical bounds on operators}

Here we list the limits on the 22 Wilson coefficients, obtained from fits to different data sets. Table 9 shows the results of our global fit, table 7 corresponds to our single top fit, and table 8 shows a fit of observables in top pair production only.

We also show a comparison of the bounds obtained from fits to top-pair production, single top production, and from the full global fit in figure 14. 


\begin{tabular}{|c|cc|}
\hline Operator & $68 \% \mathrm{CL}$ & $95 \% \mathrm{CL}$ \\
\hline$C_{t G}$ & -- & {$[-5.68,4.00]$} \\
$C_{Q q}^{38}$ & -- & {$[-0.78,0.74]$} \\
$C_{Q q}^{31}$ & -- & {$[-0.49,0.11]$} \\
$C_{b W}$ & {$[-1.84,1.68]$} & {$[-2.80,2.80]$} \\
$C_{t W}$ & {$[-0.32,0.23]$} & {$[-0.47,0.47]$} \\
$C_{t Z}$ & -- & {$[-9.40,9.40]$} \\
$C_{\phi t}$ & -- & {$[-51.50,22.50]$} \\
$C_{\phi t b}$ & {$[-5.94,5.94]$} & {$[-9.18,8.82]$} \\
$C_{\phi Q}^{3}$ & -- & {$[-4.70,1.30]$} \\
$C_{\phi Q}^{-}$ & -- & {$[-36.00,12.00]$} \\
\hline
\end{tabular}

Table 7. Bounds on the Wilson coefficients $C_{i}$ in units of $(\mathrm{TeV} / \Lambda)^{2}$ at $68 \%$ and $95 \%$ confidence level from our single top fit, corresponding to figure 11.

\begin{tabular}{|c|cc|}
\hline Operator & $68 \% \mathrm{CL}$ & $95 \% \mathrm{CL}$ \\
\hline$C_{t G}$ & {$[0.30,0.74]$} & {$[-0.03,0.82]$} \\
$C_{Q q}^{18}$ & {$[-0.79,0.15]$} & {$[-1.11,0.49]$} \\
$C_{Q q}^{38}$ & {$[-0.49,0.73]$} & {$[-0.84,1.16]$} \\
$C_{t q}^{8}$ & {$[-1.21,-0.09]$} & {$[-1.37,0.47]$} \\
$C_{Q u}^{8}$ & {$[-1.51,-0.09]$} & {$[-1.91,0.44]$} \\
$C_{Q d}^{8}$ & {$[-2.09,0.15]$} & {$[-2.44,1.24]$} \\
$C_{t u}^{8}$ & {$[-1.16,0.15]$} & {$[-1.48,0.65]$} \\
$C_{t d}^{8}$ & {$[-1.40,0.52]$} & {$[-1.93,1.16]$} \\
$C_{Q q}^{11}$ & {$[-0.38,0.09]$} & {$[-0.47,0.30]$} \\
$C_{Q q}^{31}$ & {$[-0.18,0.29]$} & {$[-0.34,0.42]$} \\
$C_{t q}^{1}$ & {$[-0.27,0.21]$} & {$[-0.39,0.37]$} \\
$C_{Q u}^{1}$ & {$[-0.47,0.09]$} & {$[-0.62,0.27]$} \\
$C_{Q d}^{1}$ & {$[-0.41,0.37]$} & {$[-0.66,0.58]$} \\
$C_{t u}^{1}$ & {$[-0.35,0.15]$} & {$[-0.47,0.34]$} \\
$C_{t d}^{1}$ & {$[-0.41,0.35]$} & {$[-0.58,0.63]$} \\
\hline
\end{tabular}

Table 8. Bounds on the Wilson coefficients $C_{i}$ in units of $(\mathrm{TeV} / \Lambda)^{2}$ at $68 \%$ and $95 \%$ confidence level from a global fit to observables in top pair production. 


\begin{tabular}{|c|cc|c|}
\hline Operator & $68 \% \mathrm{CL}$ & $95 \% \mathrm{CL}$ & $95 \% \mathrm{CL}, \delta_{\mathrm{th}} / 2$ \\
\hline$C_{t G}$ & {$[0.30,0.74]$} & {$[-0.02,0.82]$} & {$[0.24,0.57]$} \\
$C_{Q q}^{18}$ & {$[-0.68,0.20]$} & {$[-1.00,0.52]$} & {$[-0.76,0.12]$} \\
$C_{Q q}^{38}$ & {$[-0.26,0.58]$} & {$[-0.62,0.74]$} & {$[-0.42,0.54]$} \\
$C_{t q}^{8}$ & {$[-1.00,0.04]$} & {$[-1.32,0.44]$} & {$[-1.08,0.04]$} \\
$C_{Q u}^{8}$ & {$[-1.40,-0.12]$} & {$[-1.72,0.52]$} & {$[-1.32,-0.04]$} \\
$C_{Q d}^{8}$ & {$[-1.88,0.12]$} & {$[-2.20,1.08]$} & {$[-1.72,0.04]$} \\
$C_{t u}^{8}$ & {$[-1.16,0.04]$} & {$[-1.48,0.52]$} & {$[-1.16,0.04]$} \\
$C_{t d}^{8}$ & {$[-1.40,0.36]$} & {$[-1.88,1.00]$} & {$[-1.48,0.28]$} \\
$C_{Q q}^{11}$ & {$[-0.22,0.26]$} & {$[-0.38,0.42]$} & {$[-0.22,0.22]$} \\
$C_{Q q}^{31}$ & {$[-0.25,0.05]$} & {$[-0.39,0.11]$} & {$[-0.23,0.09]$} \\
$C_{t q}^{1}$ & {$[-0.22,0.22]$} & {$[-0.38,0.38]$} & {$[-0.22,0.22]$} \\
$C_{Q u}^{1}$ & {$[-0.26,0.26]$} & {$[-0.42,0.42]$} & {$[-0.30,0.22]$} \\
$C_{Q d}^{1}$ & {$[-0.38,0.38]$} & {$[-0.62,0.62]$} & {$[-0.34,0.38]$} \\
$C_{t u}^{1}$ & {$[-0.26,0.26]$} & {$[-0.42,0.42]$} & {$[-0.26,0.30]$} \\
$C_{t d}^{1}$ & {$[-0.34,0.38]$} & {$[-0.62,0.58]$} & {$[-0.30,0.38]$} \\
$C_{b W}$ & {$[-1.68,1.68]$} & {$[-2.80,2.64]$} & {$[-2.16,2.32]$} \\
$C_{t W}$ & {$[-0.23,0.26]$} & {$[-0.38,0.47]$} & {$[-0.26,0.38]$} \\
$C_{t Z}$ & {$[-2.30,2.30]$} & {$[-3.10,3.30]$} & {$[-2.90,2.50]^{*}$} \\
$C_{\phi t}$ & {$[-16.75,3.25]$} & {$[-20.75,8.75]$} & {$[-19.38,5.83]^{*}$} \\
$C_{\phi t b}$ & {$[-5.58,5.58]$} & {$[-8.46,8.82]$} & {$[-7.02,6.66]$} \\
$C_{\phi Q}^{3}$ & {$[-2.66,0.34]$} & {$[-3.98,0.94]$} & {$[-2.30,0.34]$} \\
$C_{\phi Q}^{-}$ & {$[-3.98,7.28]$} & {$[-5.78,13.12]$} & {$[-4.80,8.80]$} \\
\hline
\end{tabular}

Table 9. Bounds on the Wilson coefficients $C_{i}$ in units of $(\mathrm{TeV} / \Lambda)^{2}$ at $68 \%$ and $95 \%$ confidence level from our full global top fit, corresponding to figure 13. The asterisk marks non-Gaussian effects for which we quote conservative envelopes of the likelihood. The label $\delta_{\mathrm{th}} / 2$ stands for the fit with halved theoretical uncertainties. 
Run II, ATLAS+CMS, $68 \%$ and $95 \%$ C.L

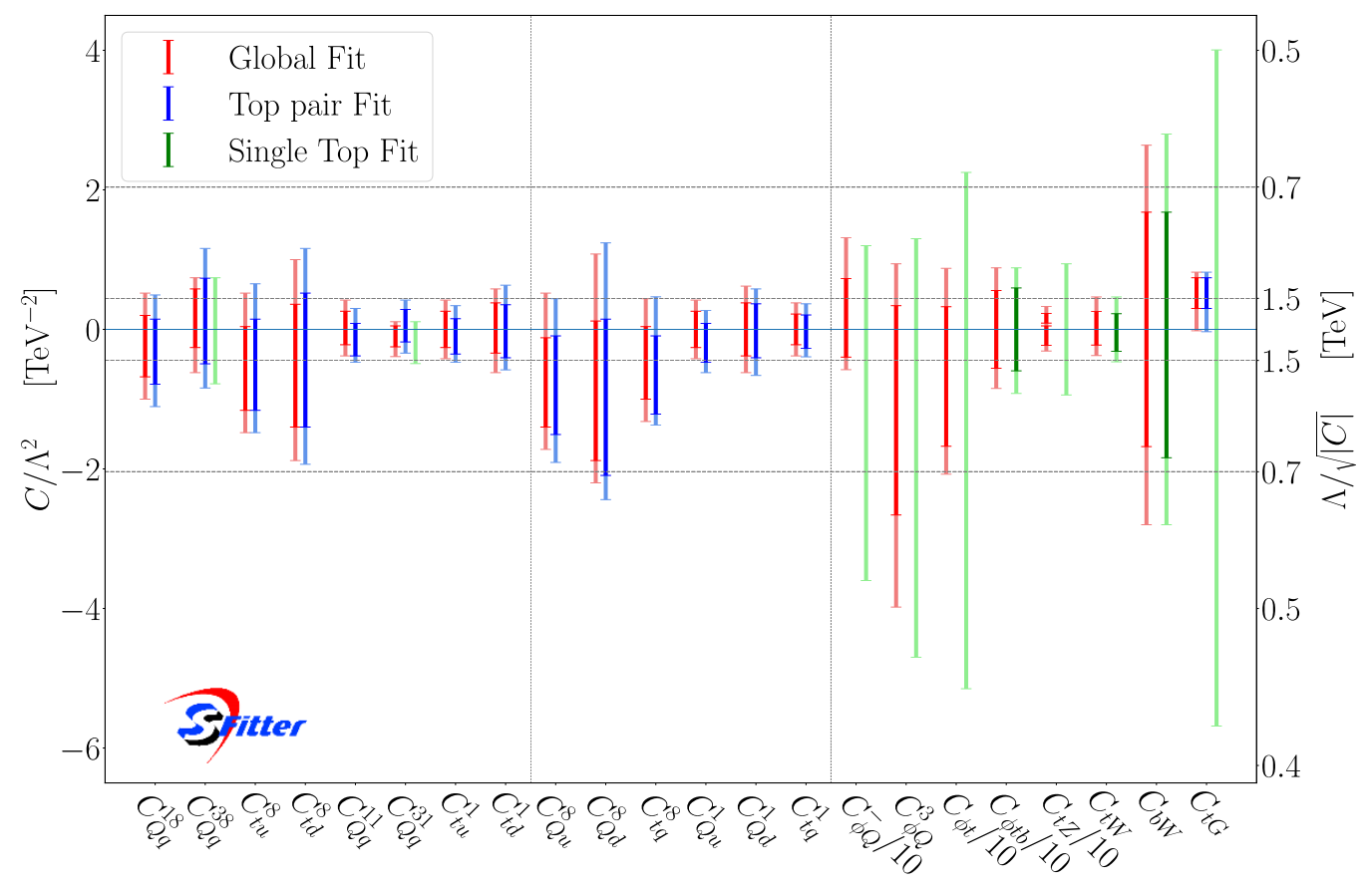

Figure 14. $95 \%$ and $68 \%$ CL bounds on top operators global fits to top pair production measurements (blue), single top (green) and to the full data set from tables 5 and 6 (red).

Open Access. This article is distributed under the terms of the Creative Commons Attribution License (CC-BY 4.0), which permits any use, distribution and reproduction in any medium, provided the original author(s) and source are credited.

\section{References}

[1] W. Buchmüller and D. Wyler, Effective Lagrangian Analysis of New Interactions and Flavor Conservation, Nucl. Phys. B 268 (1986) 621 [INSPIRE].

[2] C.N. Leung, S.T. Love and S. Rao, Low-Energy Manifestations of a New Interaction Scale: Operator Analysis, Z. Phys. C 31 (1986) 433 [InSPIRE].

[3] C. Degrande et al., Effective Field Theory: A Modern Approach to Anomalous Couplings, Annals Phys. 335 (2013) 21 [arXiv:1205.4231] [INSPIRE].

[4] I. Brivio and M. Trott, The Standard Model as an Effective Field Theory, Phys. Rept. 793 (2019) 1 [arXiv: 1706 .08945] [INSPIRE].

[5] F. Maltoni, L. Mantani and K. Mimasu, Top-quark electroweak interactions at high energy, JHEP 10 (2019) 004 [arXiv: 1904.05637] [InSPIRE].

[6] K. Hagiwara, R.D. Peccei, D. Zeppenfeld and K. Hikasa, Probing the Weak Boson Sector in $e^{+} e^{-} \rightarrow W^{+} W^{-}$, Nucl. Phys. B 282 (1987) 253 [INSPIRE].

[7] M.C. Gonzalez-Garcia, Anomalous Higgs couplings, Int. J. Mod. Phys. A 14 (1999) 3121 [hep-ph/9902321] [INSPIRE]. 
[8] A. Butter, O.J.P. Éboli, J. Gonzalez-Fraile, M.C. Gonzalez-Garcia, T. Plehn and M. Rauch, The Gauge-Higgs Legacy of the LHC Run I, JHEP 07 (2016) 152 [arXiv:1604.03105] [INSPIRE].

[9] J. Brehmer, K. Cranmer, F. Kling and T. Plehn, Better Higgs boson measurements through information geometry, Phys. Rev. D 95 (2017) 073002 [arXiv:1612.05261] [INSPIRE].

[10] J. de Blas et al., Electroweak precision observables and Higgs-boson signal strengths in the Standard Model and beyond: present and future, JHEP 12 (2016) 135 [arXiv:1608.01509] [INSPIRE].

[11] J. Ellis, C.W. Murphy, V. Sanz and T. You, Updated Global SMEFT Fit to Higgs, Diboson and Electroweak Data, JHEP 06 (2018) 146 [arXiv:1803.03252] [INSPIRE].

[12] E. da Silva Almeida, A. Alves, N. Rosa Agostinho, O.J.P. Éboli and M.C. Gonzalez-Garcia, Electroweak Sector Under Scrutiny: A Combined Analysis of LHC and Electroweak Precision Data, Phys. Rev. D 99 (2019) 033001 [arXiv:1812.01009] [INSPIRE].

[13] A. Biekötter, T. Corbett and T. Plehn, The Gauge-Higgs Legacy of the LHC Run II, SciPost Phys. 6 (2019) 064 [arXiv: 1812.07587] [inSPIRE].

[14] S. Kraml, T.Q. Loc, D.T. Nhung and L.D. Ninh, Constraining new physics from Higgs measurements with Lilith: update to LHC Run 2 results, SciPost Phys. 7 (2019) 052 [arXiv: 1908.03952] [INSPIRE].

[15] C. Degrande, J.-M. Gerard, C. Grojean, F. Maltoni and G. Servant, Non-resonant New Physics in Top Pair Production at Hadron Colliders, JHEP 03 (2011) 125 [arXiv: 1010.6304] [INSPIRE].

[16] C. Zhang and S. Willenbrock, Effective-Field-Theory Approach to Top-Quark Production and Decay, Phys. Rev. D 83 (2011) 034006 [arXiv:1008.3869] [INSPIRE].

[17] N. Greiner, S. Willenbrock and C. Zhang, Effective Field Theory for Nonstandard Top Quark Couplings, Phys. Lett. B 704 (2011) 218 [arXiv:1104.3122] [INSPIRE].

[18] Z. Hioki and K. Ohkuma, Latest constraint on nonstandard top-gluon couplings at hadron colliders and its future prospect, Phys. Rev. D 88 (2013) 017503 [arXiv:1306.5387] [INSPIRE].

[19] A. Buckley et al., Global fit of top quark effective theory to data, Phys. Rev. D 92 (2015) 091501 [arXiv: 1506.08845] [INSPIRE].

[20] A. Buckley et al., Constraining top quark effective theory in the LHC Run II era, JHEP 04 (2016) 015 [arXiv: 1512.03360] [inSPIRE].

[21] Q.-H. Cao, B. Yan, J.-H. Yu and C. Zhang, A General Analysis of Wtb anomalous Couplings, Chin. Phys. C 41 (2017) 063101 [arXiv:1504.03785] [InSPIRE].

[22] C. Englert, L. Moore, K. Nordström and M. Russell, Giving top quark effective operators a boost, Phys. Lett. B 763 (2016) 9 [arXiv:1607.04304] [InSPIRE].

[23] N.P. Hartland et al., A Monte Carlo global analysis of the Standard Model Effective Field Theory: the top quark sector, JHEP 04 (2019) 100 [arXiv:1901.05965] [INSPIRE].

[24] J. D'Hondt, A. Mariotti, K. Mimasu, S. Moortgat and C. Zhang, Learning to pinpoint

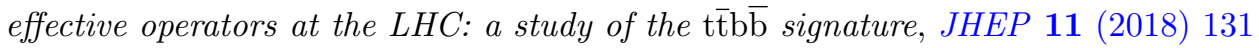
[arXiv: 1807.02130] [INSPIRE].

[25] G. Durieux, M. Perelló, M. Vos and C. Zhang, Global and optimal probes for the top-quark effective field theory at future lepton colliders, JHEP 10 (2018) 168 [arXiv:1807.02121] [INSPIRE]. 
[26] G. Durieux et al., The electro-weak couplings of the top and bottom quarks - global fit and future prospects, arXiv:1907.10619 [INSPIRE].

[27] S. Bißmann, J. Erdmann, C. Grunwald, G. Hiller and K. Kröninger, Constraining top-quark couplings combining top-quark and B decay observables, arXiv:1909.13632 [INSPIRE].

[28] D.E. Morrissey, T. Plehn and T.M.P. Tait, Physics searches at the LHC, Phys. Rept. 515 (2012) 1 [arXiv:0912.3259] [INSPIRE].

[29] J.A. Aguilar-Saavedra, B. Fuks and M.L. Mangano, Pinning down top dipole moments with ultra-boosted tops, Phys. Rev. D 91 (2015) 094021 [arXiv:1412.6654] [INSPIRE].

[30] M. Farina, C. Mondino, D. Pappadopulo and J.T. Ruderman, New Physics from High Energy Tops, JHEP 01 (2019) 231 [arXiv:1811.04084] [INSPIRE].

[31] M. Bauer, F. Goertz, U. Haisch, T. Pfoh and S. Westhoff, Top-Quark Forward-Backward Asymmetry in Randall-Sundrum Models Beyond the Leading Order, JHEP 11 (2010) 039 [arXiv: 1008.0742] [INSPIRE].

[32] C. Delaunay, O. Gedalia, Y. Hochberg, G. Perez and Y. Soreq, Implications of the CDF t $t \bar{t}$ Forward-Backward Asymmetry for Hard Top Physics, JHEP 08 (2011) 031 [arXiv: 1103.2297] [INSPIRE].

[33] M.P. Rosello and M. Vos, Constraints on four-fermion interactions from the $t \bar{t}$ charge asymmetry at hadron colliders, Eur. Phys. J. C 76 (2016) 200 [arXiv:1512.07542] [INSPIRE].

[34] J.A. Aguilar-Saavedra, Effective four-fermion operators in top physics: A Roadmap, Nucl. Phys. B 843 (2011) 638 [Erratum ibid. B 851 (2011) 443] [arXiv:1008.3562] [INSPIRE].

[35] C. Zhang, Effective field theory approach to top-quark decay at next-to-leading order in QCD, Phys. Rev. D 90 (2014) 014008 [arXiv: 1404.1264] [INSPIRE].

[36] J. Alwall et al., The automated computation of tree-level and next-to-leading order differential cross sections and their matching to parton shower simulations, JHEP $\mathbf{0 7}$ (2014) 079 [arXiv: 1405.0301] [INSPIRE].

[37] C. Degrande, Automatic evaluation of UV and R2 terms for beyond the Standard Model Lagrangians: a proof-of-principle, Comput. Phys. Commun. 197 (2015) 239 [arXiv: 1406.3030] [INSPIRE].

[38] R. Lafaye, T. Plehn, M. Rauch and D. Zerwas, Measuring Supersymmetry, Eur. Phys. J. C 54 (2008) 617 [arXiv: 0709.3985] [INSPIRE].

[39] R. Lafaye, T. Plehn, M. Rauch, D. Zerwas and M. Dührssen, Measuring the Higgs Sector, JHEP 08 (2009) 009 [arXiv:0904.3866] [INSPIRE].

[40] D. Barducci et al., Interpreting top-quark LHC measurements in the standard-model effective field theory, arXiv: 1802.07237 [INSPIRE].

[41] G. Durieux, F. Maltoni and C. Zhang, Global approach to top-quark flavor-changing interactions, Phys. Rev. D 91 (2015) 074017 [arXiv:1412.7166] [InSPIRE].

[42] C. Degrande, F. Maltoni, J. Wang and C. Zhang, Automatic computations at next-to-leading order in QCD for top-quark flavor-changing neutral processes, Phys. Rev. D 91 (2015) 034024 [arXiv:1412.5594] [INSPIRE].

[43] D. Buarque Franzosi and C. Zhang, Probing the top-quark chromomagnetic dipole moment at next-to-leading order in QCD, Phys. Rev. D 91 (2015) 114010 [arXiv:1503.08841] [INSPIRE]. 
[44] B. Grzadkowski, M. Iskrzynski, M. Misiak and J. Rosiek, Dimension-Six Terms in the Standard Model Lagrangian, JHEP 10 (2010) 085 [arXiv: 1008.4884] [INSPIRE].

[45] F. Krauss, S. Kuttimalai and T. Plehn, LHC multijet events as a probe for anomalous dimension-six gluon interactions, Phys. Rev. D 95 (2017) 035024 [arXiv:1611.00767] [INSPIRE].

[46] V. Hirschi, F. Maltoni, I. Tsinikos and E. Vryonidou, Constraining anomalous gluon self-interactions at the LHC: a reappraisal, JHEP 07 (2018) 093 [arXiv:1806.04696] [INSPIRE].

[47] C. Englert, M. Russell and C.D. White, Effective Field Theory in the top sector: do multijets help?, Phys. Rev. D 99 (2019) 035019 [arXiv:1809.09744] [INSPIRE].

[48] P. Ferrario and G. Rodrigo, Massive color-octet bosons and the charge asymmetries of top quarks at hadron colliders, Phys. Rev. D 78 (2008) 094018 [arXiv:0809.3354] [INSPIRE].

[49] C. Degrande, F. Maltoni, K. Mimasu, E. Vryonidou and C. Zhang, Single-top associated production with a $Z$ or $H$ boson at the LHC: the SMEFT interpretation, JHEP 10 (2018) 005 [arXiv: 1804.07773] [INSPIRE].

[50] C. Zhang, Single Top Production at Next-to-Leading Order in the Standard Model Effective Field Theory, Phys. Rev. Lett. 116 (2016) 162002 [arXiv:1601.06163] [InSPIRE].

[51] R. Boughezal, C.-Y. Chen, F. Petriello and D. Wiegand, Top quark decay at next-to-leading order in the Standard Model Effective Field Theory, Phys. Rev. D 100 (2019) 056023 [arXiv: 1907.00997] [INSPIRE].

[52] F. Maltoni, E. Vryonidou and C. Zhang, Higgs production in association with a top-antitop pair in the Standard Model Effective Field Theory at NLO in QCD, JHEP 10 (2016) 123 [arXiv: 1607.05330] [INSPIRE].

[53] O. Bessidskaia Bylund, F. Maltoni, I. Tsinikos, E. Vryonidou and C. Zhang, Probing top quark neutral couplings in the Standard Model Effective Field Theory at NLO in QCD, JHEP 05 (2016) 052 [arXiv: 1601.08193] [InSPIRE].

[54] M. Schulze and Y. Soreq, Pinning down electroweak dipole operators of the top quark, Eur. Phys. J. C 76 (2016) 466 [arXiv:1603.08911] [INSPIRE].

[55] CMS collaboration, Measurement of the t-tbar production cross section in the e-mu channel in proton-proton collisions at $\sqrt{s}=7$ and $8 \mathrm{TeV}$, JHEP 08 (2016) 029 [arXiv:1603.02303] [INSPIRE].

[56] M. Czakon and A. Mitov, Top++: A Program for the Calculation of the Top-Pair Cross-Section at Hadron Colliders, Comput. Phys. Commun. 185 (2014) 2930 [arXiv:1112.5675] [INSPIRE].

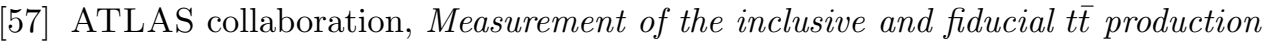
cross-sections in the lepton+jets channel in pp collisions at $\sqrt{s}=8$ TeV with the ATLAS detector, Eur. Phys. J. C 78 (2018) 487 [arXiv:1712.06857] [INSPIRE].

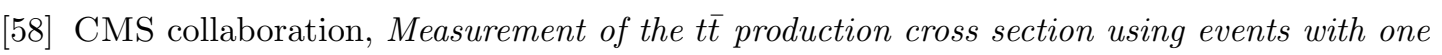
lepton and at least one jet in pp collisions at $\sqrt{s}=13 \mathrm{TeV}$, JHEP 09 (2017) 051 [arXiv: 1701.06228] [INSPIRE].

[59] CMS collaboration, Measurement of the $\mathrm{t} \overline{\mathrm{t}}$ production cross section, the top quark mass and the strong coupling constant using dilepton events in pp collisions at $\sqrt{s}=13 \mathrm{TeV}$, Eur. Phys. J. C 79 (2019) 368 [arXiv:1812.10505] [InSPIRE]. 


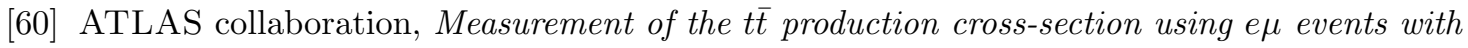
b-tagged jets in pp collisions at $\sqrt{s}=13 \mathrm{TeV}$ with the ATLAS detector, Phys. Lett. B 761 (2016) 136 [Erratum ibid. B $\mathbf{7 7 2}$ (2017) 879] [arXiv:1606.02699] [INSPIRE].

[61] ATLAS collaboration, Measurements of top-quark pair differential cross-sections in the lepton+jets channel in pp collisions at $\sqrt{s}=8 \mathrm{TeV}$ using the ATLAS detector, Eur. Phys. J. C 76 (2016) 538 [arXiv:1511.04716] [InSPIRE].

[62] M. Czakon, D. Heymes and A. Mitov, fastNLO tables for NNLO top-quark pair differential distributions, arXiv:1704.08551 [INSPIRE].

[63] M. Czakon, D. Heymes and A. Mitov, High-precision differential predictions for top-quark pairs at the LHC, Phys. Rev. Lett. 116 (2016) 082003 [arXiv:1511.00549] [INSPIRE].

[64] M. Czakon, D. Heymes and A. Mitov, Dynamical scales for multi-TeV top-pair production at the LHC, JHEP 04 (2017) 071 [arXiv: 1606. 03350] [INSPIRE].

[65] CMS collaboration, Measurement of the differential cross section for top quark pair production in pp collisions at $\sqrt{s}=8 \mathrm{TeV}$, Eur. Phys. J. C 75 (2015) 542 [arXiv: 1505. 04480] [INSPIRE].

[66] CMS collaboration, Measurement of double-differential cross sections for top quark pair production in pp collisions at $\sqrt{s}=8 \mathrm{TeV}$ and impact on parton distribution functions, Eur. Phys. J. C 77 (2017) 459 [arXiv:1703.01630] [INSPIRE].

[67] M. Czakon, A. Mitov and A. Papanastasiou, to appear.

[68] CMS collaboration, Measurement of the integrated and differential t $\bar{t}$ production cross sections for high-p top quarks in pp collisions at $\sqrt{s}=8 \mathrm{TeV}$, Phys. Rev. D 94 (2016) 072002 [arXiv: 1605.00116] [INSPIRE].

[69] CMS collaboration, Measurement of differential cross sections for top quark pair production using the lepton+jets final state in proton-proton collisions at $13 \mathrm{TeV}$, Phys. Rev. D 95 (2017) 092001 [arXiv:1610.04191] [INSPIRE].

[70] CMS collaboration, Measurement of differential cross sections for the production of top quark pairs and of additional jets in lepton+jets events from pp collisions at $\sqrt{s}=13 \mathrm{TeV}$, Phys. Rev. D 97 (2018) 112003 [arXiv:1803.08856] [INSPIRE].

[71] CMS collaboration, Measurement of normalized differential $\mathrm{t} \overline{\mathrm{t}}$ cross sections in the dilepton channel from pp collisions at $\sqrt{s}=13 \mathrm{TeV}$, JHEP 04 (2018) 060 [arXiv:1708.07638] [INSPIRE].

[72] CMS collaboration, Measurements of $\mathrm{t} \overline{\mathrm{t}}$ differential cross sections in proton-proton collisions at $\sqrt{s}=13 \mathrm{TeV}$ using events containing two leptons, JHEP 02 (2019) 149 [arXiv: 1811.06625] [INSPIRE].

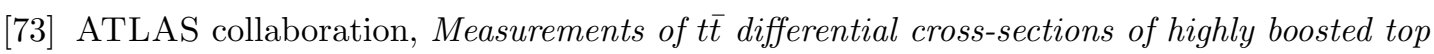
quarks decaying to all-hadronic final states in pp collisions at $\sqrt{s}=13 \mathrm{TeV}$ using the ATLAS detector, Phys. Rev. D 98 (2018) 012003 [arXiv:1801.02052] [INSPIRE].

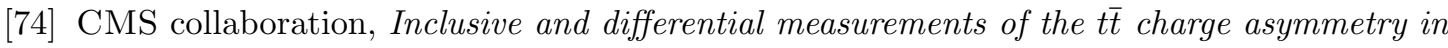
pp collisions at $\sqrt{s}=8 \mathrm{TeV}$, Phys. Lett. B 757 (2016) 154 [arXiv:1507.03119] [INSPIRE].

[75] M. Czakon, D. Heymes, A. Mitov, D. Pagani, I. Tsinikos and M. Zaro, Top-quark charge asymmetry at the LHC and Tevatron through NNLO QCD and NLO EW, Phys. Rev. D 98 (2018) 014003 [arXiv: 1711.03945] [inSPIRE].

[76] CMS collaboration, Measurements of $t \bar{t}$ charge asymmetry using dilepton final states in pp collisions at $\sqrt{s}=8 \mathrm{TeV}$, Phys. Lett. B 760 (2016) 365 [arXiv:1603.06221] [INSPIRE]. 
[77] ATLAS collaboration, Measurement of the charge asymmetry in top-quark pair production in the lepton-plus-jets final state in pp collision data at $\sqrt{s}=8 \mathrm{TeV}$ with the ATLAS detector, Eur. Phys. J. C 76 (2016) 87 [Erratum ibid. C 77 (2017) 564] [arXiv: 1509.02358] [INSPIRE].

[78] ATLAS collaboration, Measurements of the charge asymmetry in top-quark pair production in the dilepton final state at $\sqrt{s}=8 \mathrm{TeV}$ with the ATLAS detector, Phys. Rev. D 94 (2016) 032006 [arXiv: 1604.05538] [INSPIRE].

[79] ATLAS collaboration, Inclusive and differential measurement of the charge asymmetry in $t \bar{t}$ events at $13 \mathrm{TeV}$ with the ATLAS detector, ATLAS-CONF-2019-026 (2019).

[80] CMS collaboration, Measurement of top quark pair production in association with a $Z$ boson in proton-proton collisions at $\sqrt{s}=13 \mathrm{TeV}$, arXiv:1907.11270 [INSPIRE].

[81] A. Kulesza, L. Motyka, D. Schwartländer, T. Stebel and V. Theeuwes, Associated production of a top quark pair with a heavy electroweak gauge boson at $N L O+N N L L$ accuracy, Eur. Phys. J. C 79 (2019) 249 [arXiv: 1812.08622] [INSPIRE].

[82] ATLAS collaboration, Measurement of the $t \bar{t} Z$ and $t \bar{t} W$ production cross sections in multilepton final states using $3.2 \mathrm{fb}^{-1}$ of pp collisions at $\sqrt{\mathrm{s}}=13 \mathrm{TeV}$ with the ATLAS detector, Eur. Phys. J. C 77 (2017) 40 [arXiv:1609.01599] [INSPIRE].

[83] CMS collaboration, Measurement of the cross section for top quark pair production in association with a $W$ or $Z$ boson in proton-proton collisions at $\sqrt{s}=13 \mathrm{TeV}$, JHEP 08 (2018) 011 [arXiv: 1711.02547] [INSPIRE].

[84] CMS collaboration, Measurement of the Single-Top-Quark t-Channel Cross Section in pp Collisions at $\sqrt{s}=7 \mathrm{TeV}$, JHEP 12 (2012) 035 [arXiv:1209.4533] [INSPIRE].

[85] ATLAS collaboration, Comprehensive measurements of t-channel single top-quark production cross sections at $\sqrt{s}=7 \mathrm{TeV}$ with the ATLAS detector, Phys. Rev. D 90 (2014) 112006 [arXiv: 1406.7844] [INSPIRE].

[86] ATLAS collaboration, Fiducial, total and differential cross-section measurements of t-channel single top-quark production in pp collisions at $8 \mathrm{TeV}$ using data collected by the ATLAS detector, Eur. Phys. J. C 77 (2017) 531 [arXiv:1702.02859] [INSPIRE].

[87] CMS collaboration, Measurement of the t-channel single-top-quark production cross section and of the $\left|V_{t b}\right|$ CKM matrix element in pp collisions at $\sqrt{s}=8$ TeV, JHEP 06 (2014) 090 [arXiv: 1403.7366] [INSPIRE].

[88] ATLAS collaboration, Measurement of the inclusive cross-sections of single top-quark and top-antiquark t-channel production in pp collisions at $\sqrt{s}=13 \mathrm{TeV}$ with the ATLAS detector, JHEP 04 (2017) 086 [arXiv: 1609.03920] [INSPIRE].

[89] E.L. Berger, J. Gao, C.P. Yuan and H.X. Zhu, NNLO QCD Corrections to t-channel Single Top-Quark Production and Decay, Phys. Rev. D 94 (2016) 071501 [arXiv:1606.08463] [INSPIRE].

[90] CMS collaboration, Measurement of the inclusive cross section of single top quark production in the $t$ channel at $\sqrt{s}=13 \mathrm{TeV}$, CMS-PAS-TOP-16-003 (2016).

[91] CMS collaboration, Search for s channel single top quark production in pp collisions at $\sqrt{s}=7$ and $8 \mathrm{TeV}$, JHEP 09 (2016) 027 [arXiv: 1603.02555] [INSPIRE].

[92] ATLAS collaboration, Evidence for single top-quark production in the s-channel in proton-proton collisions at $\sqrt{s}=8 \mathrm{TeV}$ with the ATLAS detector using the Matrix Element Method, Phys. Lett. B 756 (2016) 228 [arXiv:1511.05980] [INSPIRE]. 
[93] ATLAS collaboration, Evidence for the associated production of a $W$ boson and a top quark in ATLAS at $\sqrt{s}=7 \mathrm{TeV}$, Phys. Lett. B 716 (2012) 142 [arXiv:1205.5764] [InSPIRE].

[94] CMS collaboration, Evidence for Associated Production of a Single Top Quark and W Boson in pp Collisions at $\sqrt{s}=7 \mathrm{TeV}$, Phys. Rev. Lett. 110 (2013) 022003 [arXiv: 1209.3489] [INSPIRE].

[95] ATLAS collaboration, Measurement of the production cross-section of a single top quark in association with a $W$ boson at $8 \mathrm{TeV}$ with the ATLAS experiment, JHEP 01 (2016) 064 [arXiv: 1510.03752] [INSPIRE].

[96] CMS collaboration, Observation of the associated production of a single top quark and a $W$ boson in pp collisions at $\sqrt{s}=8 \mathrm{TeV}$, Phys. Rev. Lett. 112 (2014) 231802 [arXiv: 1401.2942] [INSPIRE].

[97] ATLAS collaboration, Measurement of the cross-section for producing a $W$ boson in association with a single top quark in pp collisions at $\sqrt{s}=13 \mathrm{TeV}$ with ATLAS, JHEP 01 (2018) 063 [arXiv: 1612.07231] [inSPIRE].

[98] CMS collaboration, Measurement of the production cross section for single top quarks in association with $W$ bosons in proton-proton collisions at $\sqrt{s}=13$ TeV, JHEP 10 (2018) 117 [arXiv: 1805. 07399] [INSPIRE].

[99] ATLAS collaboration, Measurement of the production cross-section of a single top quark in association with a $Z$ boson in proton-proton collisions at $13 \mathrm{TeV}$ with the ATLAS detector, Phys. Lett. B 780 (2018) 557 [arXiv:1710.03659] [INSPIRE].

[100] ATLAS collaboration, Measurement of the $W$ boson polarization in top quark decays with the ATLAS detector, JHEP 06 (2012) 088 [arXiv:1205.2484] [INSPIRE].

[101] CMS collaboration, Measurement of the W-Boson Helicity in Top-Quark decays from $t \bar{t}$ Production in Lepton + Jets Events in pp Collisions at $\sqrt{s}=7$ TeV, JHEP 10 (2013) 167 [arXiv: 1308.3879] [INSPIRE].

[102] ATLAS collaboration, Measurement of the $W$ boson polarisation in t $\bar{t}$ events from $p p$ collisions at $\sqrt{s}=8 \mathrm{TeV}$ in the lepton + jets channel with ATLAS, Eur. Phys. J. C 77 (2017) 264 [Erratum ibid. C 79 (2019) 19] [arXiv:1612.02577] [INSPIRE].

[103] CMS collaboration, Measurement of the $W$ boson helicity fractions in the decays of top quark pairs to lepton + jets final states produced in pp collisions at $\sqrt{s}=8 \mathrm{TeV}$, Phys. Lett. B 762 (2016) 512 [arXiv: 1605.09047] [INSPIRE].

[104] J.A. Aguilar-Saavedra, C. Degrande and S. Khatibi, Single top polarisation as a window to new physics, Phys. Lett. B 769 (2017) 498 [arXiv:1701.05900] [INSPIRE].

[105] M. de Beurs, E. Laenen, M. Vreeswijk and E. Vryonidou, Effective operators in t-channel single top production and decay, Eur. Phys. J. C 78 (2018) 919 [arXiv:1807.03576] [INSPIRE].

[106] T. Neumann and Z.E. Sullivan, Off-Shell Single-Top-Quark Production in the Standard Model Effective Field Theory, JHEP 06 (2019) 022 [arXiv: 1903.11023] [INSPIRE].

[107] J. Brehmer, S. Dawson, S. Homiller, F. Kling and T. Plehn, Benchmarking simplified template cross sections in WH production, JHEP 11 (2019) 034 [arXiv:1908.06980] [INSPIRE].

[108] G. Bevilacqua, M. Czakon, A. van Hameren, C.G. Papadopoulos and M. Worek, Complete off-shell effects in top quark pair hadroproduction with leptonic decay at next-to-leading order, JHEP 02 (2011) 083 [arXiv: 1012.4230] [INSPIRE]. 
[109] A. Denner and M. Pellen, NLO electroweak corrections to off-shell top-antitop production with leptonic decays at the LHC, JHEP 08 (2016) 155 [arXiv: 1607.05571] [INSPIRE].

[110] A. Denner and M. Pellen, Off-shell production of top-antitop pairs in the lepton+jets channel at NLO QCD, JHEP 02 (2018) 013 [arXiv:1711.10359] [INSPIRE].

[111] M. Czakon, D. Heymes, A. Mitov, D. Pagani, I. Tsinikos and M. Zaro, Top-pair production at the LHC through NNLO QCD and NLO EW, JHEP 10 (2017) 186 [arXiv:1705.04105] [INSPIRE].

[112] M. Czakon, P. Fiedler, D. Heymes and A. Mitov, NNLO QCD predictions for fully-differential top-quark pair production at the Tevatron, JHEP 05 (2016) 034 [arXiv: 1601.05375] [INSPIRE].

[113] M. Czakon et al., Resummation for (boosted) top-quark pair production at $N N L O+N N L L^{\prime}$ in QCD, JHEP 05 (2018) 149 [arXiv: 1803.07623] [INSPIRE].

[114] A. Hocker, H. Lacker, S. Laplace and F. Le Diberder, A New approach to a global fit of the CKM matrix, Eur. Phys. J. C 21 (2001) 225 [hep-ph/0104062] [INSPIRE].

[115] NNPDF collaboration, Parton distributions from high-precision collider data, Eur. Phys. J. C 77 (2017) 663 [arXiv: 1706.00428] [INSPIRE].

[116] L.A. Harland-Lang, A.D. Martin, P. Motylinski and R.S. Thorne, Parton distributions in the LHC era: MMHT 2014 PDFs, Eur. Phys. J. C 75 (2015) 204 [arXiv:1412.3989] [INSPIRE].

[117] S. Dulat et al., New parton distribution functions from a global analysis of quantum chromodynamics, Phys. Rev. D 93 (2016) 033006 [arXiv:1506.07443] [InSPIRE].

[118] P. Skands, B. Webber and J. Winter, QCD Coherence and the Top Quark Asymmetry, JHEP 07 (2012) 151 [arXiv:1205.1466] [INSPIRE]. 